\title{
Additions and Corrections to the Aphididae of Puerto Rico'
}

\author{
Clyde F. Smith, Silverio Medina Gaud, Luis F. Martorell, and \\ Mario E. Pérez-Escolar ${ }^{2}$
}

\section{INTRODUCTION}

Since the publication of the "Aphididae of Puerto Rico" (Smith, Martorell, and Pérez-Escolar (12), ${ }^{8}$ additional survey work has been done, especially during 1967 and 1969. Five additional species have been found: Capitophorus hippophaes javanicus Hille Ris Lambers, Macrosiphum rosae L., Picturaphis puertoricensis Smith, Rhopalosiphum padi L., and Tetraneura nigriabdominalis (Sasaki). Prociphilus erigeronensis (Thomas), recorded by Wolcott (18), was seen on a slide sent from the U. S. National Museum. The specimen had been intercepted by plant quarantine personnel. Rhopalosiphum rufiabdominalis (Sasaki), recorded as $R$. subterraneum Mason by Wolcott (19) also was collected. These species are redescribed and illustrated, and new host and locality records are included for the species recorded previously. The "Key to the Aphididae of Puerto Rico" has been revised. A key to the apterous Aphididae of Puerto Rico also has been prepared which, with the aid of a $10 \times$ magnifying glass, can be used in the field.

In citing host plant records ${ }^{4}$ we have followed Liogier $(8,9)$. The new name appears first, followed by an " =" sign and Britton's old name which we used in 1963 (12).

The first numbers in parentheses in the collection records indicate the year of collection, and the second numbers indicate the collection number. The specimens are in the collection of North Carolina State University at Raleigh, Puerto Rico Agricultural Experiment Station at Río Piedras, and the collection of Clyde F. Smith. To save space, initials have been used for the following collectors' names: CFS to Clyde F. Smith, CKS for Mrs.

1 Contributions from the Departments of Entomology, Agricultural Experiment Station, Mayagüez Campus, University of Puerto Rico, Río Piedras, P.R., and North Carolina State University (Journal Paper No. 3158) at Raleigh, N.C.

- Professor, Department of Entomology, North Carolina State University at Raleigh and Visiting Scientist, Agricultural Experiment Station, Mayagüez Campus, University of Puerto Rico, Río Piedras, P.R.; Assistant Entomologist, Entomologist, and Head, Department of Entomology, respectively, Agricultural Experiment Station, Mayagüez Campus, University of Puerto Rico.

8 Italic numbers in parentheses refer to Literature Cited, p. 224-5.

- We are indebted to Roy Woodbury, Botanist, Puerto Rico Agricultural Experiment Station, for aid in collecting and for identifying many of the plants. 
Clyde F. Smith, LFM for Luis F. Martorell, MEP for Mario E. Pérez, RW for Roy Woodbury, and SMG for Silverio Medina Gaud.

The following abbreviations are used in the keys and descriptions:

a.s. - antennal segments

R IV + V-rostral segments IV + V

p.t.-processus terminalis

t.ch.-tarsomere I chaetotaxy

ca.- approximately

\section{KEY TO THE APHIDIDAE OF PUERTO RICO}

1 Siphunculi conspicuous, longer than broad, usually longer than cauda; cauda never knobbed.................. 10

Siphunculi not longer than broad, may be mammiform and hairy; cauda may or may not be knobbed.............. 2

2 (1) Cauda distinctly knobbed..................... 6

Cauda not distinctly knobbed. ................. 3

3 (2) Tarsomeres I and II separate in aptera............ 4

Tarsomeres I and II fused in aptera; marginal setae long and conspicuous, 4 or more times as long as medial or pleural setae (fig. 51, 52).......... Tetraneura nigriabdominalis (Sasaki)

4 (3) Primary sensoria with a hairy fringe; siphunculi absent or in-

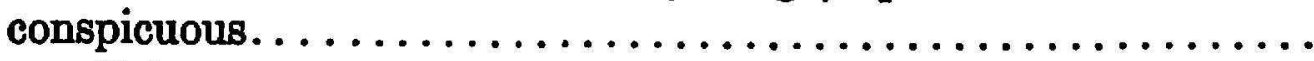

Primary sensoria nude, without a hairy fringe; siphunculi mammiform, hairy (fig. 20).... Cinara tujafilina (Del Guercio)

5 (4) R IV + V with 2 accessory setae, setae outside of lateral wax plates, cauda with 3 setae (fig. 24). Geopemphigus floccosus (Moreira)

R IV + V with 4 or more accessory setae, setae inside of lateral wax plates, cauda with ca. 12 setae (fig. 43)

Prociphilus erigeronensis (Thomas)

6 (2) Antennal sensoria annular; aptera with hornlike projections on vertex; aptera often scalelike.................. 7

Antennal sensoria oval; aptera without hornlike projections

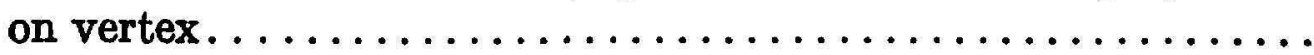

7 (6) Body of aptera scalelike, a distinct suture between the mesoand metathorax; head, prothorax, and mesothorax fused together

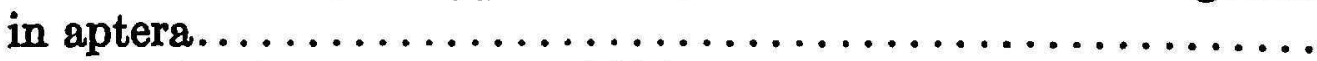

Body of aptera more aphidlike with the most distinct suture between the pro- and mesothorax; head and prothorax fused together in aptera (fig. 38)........... Oregma panicola Takahashi

8 (7) Front of aptera always with 2 horns; underside of front, near horns, with a number of normal, thin setae on small sockets (fig. 18).................... Cerataphis orchidearum (Westwood) 
Front of aptera with or without horns; underside of front with 1 to 3 pairs of short, dagger or club-shaped setae in addition to the normal setae (fig. 19)..........Cerataphis variabilis H.R.L.

9 (6) Vertex with long $(0.1)^{1}$ spinelike setae; abdomen without dorsal tubercles (fig. 50)................ Sipha flava (Forbes)

Vertex without long spinelike setae (setae 0.02); abdomen with dorsal tubercles (fig. 34)

Melanocallis kahawaluokalani (Kirkaldy)

10 (1) Antennal tubercles often well-developed; abdominal segments I and VII usually without lateral abdominal tubercles; if tubercles are present on abdominal segments I and VII, they are no longer than the tubercles on segments II and VI........ 11

Antennal tubercles never well-developed; abdominal segments I and VII with lateral abdominal tubercles which are larger than those on segments II and VI................. 42

11(10) Siphunculi with distinct reticulations (4 or more rows).... 12

Siphunculi without distinct reticulations. ........... 20

12(11) Siphunculi with cells of reticulations small (each cell about $1 / 6$ or $1 / 5$ the diameter of the reticulated area of the siphunculus).. 13

Siphunculi with cells of reticulations large (each cell about $1 / 4$ or $1 / 3$ the diameter of the reticulated area of the siphunculus). . 15

13(12) Base of siphunculi pale..................... 14 Siphunculi unicolorous, base dark (fig. 21)

Dactynotus ambrosiae (Thomas)

14(13) A.s. III subequal to siphunculus. A.s. III of aptera with 5-14 rhinaria, alate 18-27 (fig. 22).... Dactynotus erigeronensis (Thomas)

A.s. III distinctly longer than siphunculus. A.s. III of aptera with 8-21 rhinaria, alate 39-46 (fig. 23)

Dactynotus gravicornis (Patch)

15(12) Dorsum of abdomen of aptera (cleared specimens) sclerotic. 16 Dorsum of abdomen of aptera (cleared specimens) not sclerotic.

16(15) Cauda usually bearing 9 setae; vertex setae 0.02 ; on orchids (fig. 30).............Macrosiphum (Sitobion) luteum (Buckton)

Cauda usually bearing 7 setae; vertex setae 0.04 ; on Salvia and Hyptis (figs. 32, 33)

Macrosiphum (Sitobion) salviae (Bartholomew)

17(15) Siphunculi with 8 or more rows of reticulations......... 18 Siphunculi with 0 to 4 rows of reticulations, Fig. 10 Aulacorthum solani (Kalt.)

${ }^{1}$ All measurements given are in millimeters. 
18(17) Siphunculi dark.......................... 19

Siphunculi pale at base, may be dark on distal part, especially in alate (fig. 29) .............Macrosiphum euphorbiae (Thomas)

19(18) Sensoria on a.s. III, aptera 1-4, alate ca. 14 (figs. 32, 33) Macrosiphum (Sitobion) salviae (Bartholomew)

Sensoria on a.s. III, aptera 12-23, alate 42-58 (fig. 31) Macrosiphum rosae (L.)

20(11) Setae on vertex of head blunt or pointed (may be slightly

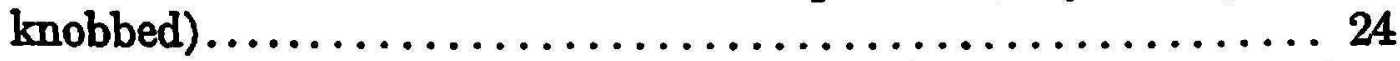

Setae on vertex of head distinctly knobbed or fan-shaped, especially in aptera............................ 21

21(20) Setae on vertex of head short, and fan-shaped; radial sector fused with media for part of its length (fig. 40)

Pentalonia nigronervosa Coquerel

Setae on vertex of head conspicuous and distinctly knobbed, especially in aptera............................. 22

22(21) T.ch. 3-3-3. On Cirsium and Polygonum.............. 23 T.ch. 5-5-5. On Fragaria (fig. 15)

Capitophorus (Pentatrichopus) minor (Forbes)

23(22) Abdominal segments VII-VIII of aptera each with 4-6 conspicuous, knobbed setae. On Cirsium (fig. 13)

Capitophorus elaeagni (Del Guercio)

Abdominal segments VII-VIII of aptera each with 2 conspicuous, knobbed setae. On Polygonum (fig. 14)

Capitophorus hippophaes subsp. javanicus H.R.L.

24(20) Frontal tubercles usually well developed, distinctly exceeding vertex................................... 25

Frontal tubercles not distinctly exceeding vertex....... 26

25(24) Dorsum of abdomen of aptera without a dark U-shaped area. 29

Dorsum of abdomen of aptera with a dark U-shaped area

(fig. 9)..........Aulacorthum (Neomyzus) circumflexum (Buckton)

26(24) Hindwing with media and cubitus; on Cruciferae........ 27

Hindwing with cubitus lacking; on Cyperaceae......... 28

27(26) Siphunculus subequal to R IV + V; alate without sensoria on a.s. IV (fig. 12)................. Brevicoryne brassicae (L.)

Siphunculus 2 times length of $\mathbf{R}$ IV $+V$, alate with sensoria on a.s. IV (fig. 28).............. Lipahis pseudobrassicae (Davis)

28(26) P.t. of aptera 1 to 1.5 times base, of alates, 1.75 to 2.0 times base (fig. 16)........................ Carolinaia caricis Wilson

P.t. of aptera 2 to 2.5 times base, of alates 2.5 to 3.0 times base (fig. 17) ..................... Carolinaia cyperi Ainslie 
29(25) Inner sides of antennal tubercles diverging, parallel or

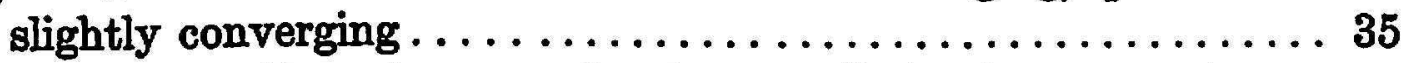

Inner sides of antennal tubercles distinctly converging or

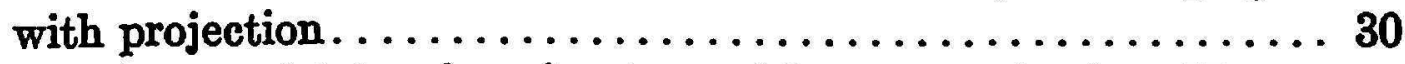
30(29) Antennal tubercles of aptera without a projection. Not on Mentha

Antennal tubercles of aptera with a projection; on Mentha (fig. 39)................... Ovatus crataegarius (Walker) 31(30) Antennae about equal to or longer than body.......... 32 Antennae distinctly shorter than length of body, easily seen without measuring (fig. 36)...............Myzus ornatus Laing 32(31) Siphunculi pale; a.s. IV and V of alate without rhinaria; hind tibiae of nymphs without spinules.............. 33

Siphunculi dark; a.s. IV and V of alate bearing rhinaria; hind tibiae of nymphs with spinules (fig. 25)

33(32) A.s. III without rhinaria (fig. 37)

Hyalomyzus jussiaeae Smith Myzus (Nectarosiphon) persicae (Sulzer)

34(33) A.s. III with 1-4 rhinaria (fig. 10). . Aulacorthum solani (Kalt.) A.s. III with 5-13 rhinaria (fig. 44)

Rhodobium porosum (Sanderson)

35(29) Radial sector of forewing not fused with media, normal; in aptera, distal 1/2 of siphunculus not distinctly darker than basal

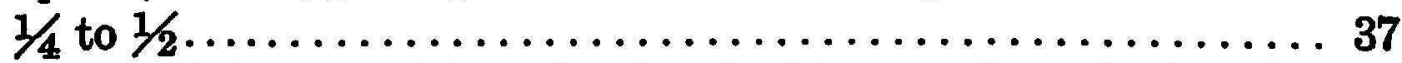

Radial sector of forewing fused with upper branch of media, forming a closed cell; in aptera, distal $1 / 2$ of siphunculus dark, basal $1 / 4$ to $1 / 2$ pale.............................. 36

36(35) Siphunculi 1.1-1.5 times cauda. Aptera dark brown to blackish (fig. 41).............Picturaphis brasiliensis (Moreira)

Siphunculi 1.7-2.0 times cauda. Aptera reddish-brown, shiny (fig. 42).................. Picturaphis puertoricensis Smith 37(35) Hindwing with media and cubitus; siphunculus of aptera not surrounded by a large sclerite.................... 38

Hindwing without medius or cubitus; siphunculus of aptera surrounded by a large conspicuous sclerite (fig. 35)

Microparsus olivei Smith and Tuatay

38(37) Siphunculi distinctly swollen on distal half . . . . . . . . 39 Siphunculi not swollen on distal half...............40

39(38) A.s. III of aptera with 6 or more rhinaria; alate with numerous rhinaria on a.s. III, IV, and V (fig. 26) 
A.s. III of aptera with 0 to 2 rhinaria; a.s. III of alate with 10 to 15 rhinaria, a.s. IV and V without rhinaria (fig. 2)

Amphorophora commelinensis Smith

40(38) Cauda bearing 3 pairs of lateral setae; vertex of head of aptera without a rectangular process.

Cauda bearing 2 pairs of lateral setae; vertex on head of aptera with a rectangular process (fig. 44)

Rhodobium porosum (Sanderson)

41(40) Siphunculi dark, basal 1/5 may be pale; antennal tubercles diverging anteriorly (fig. 1).........Acyrthosiphon bidenticola Smith

Siphunculi pale, tip (1/5) may be dusky; inner sides of antennal tubercles parallel or slightly converging (fig. 10)

Aulacorthum solani (Kalt.)

42(10) Abdomen with prominent striae (which are denticulate on the caudal margin) on each side, caudad of siphunculi (fig. 53)

Toxoptera aurantiae (Boyer de Fonscolombe)

Abdomen without such striae...................43

43(42) Siphunculi imbricated...................... 44

Siphunculi not imbricated (fig. 11)

Brachycaudus helichrysi (Kalt.)

44(43) Siphunculi cylindrical or tapering; pentagonal or hexagonal markings (if present) on dorsum of abdomen of aptera, usually outlined by more or less continuous lines.............. 50

Siphunculi swollen or clavate; pentagonal or hexagonal markings (if present) on dorsum of abdomen of aptera, outlined by broken lines, spicules or enlarged nodule-like swellings....... 45

45(44) Abdomen of aptera with distinct pentagonal markings, hindwing of alate with 2 cross-veins. . . . . . . . . . . . . . 47

Abdomen of aptera rugose but without distinct pentagonal markings; hindwing of alate with only 1 cross-vein. . . . . . . 46

46(45) P.t. of aptera 1 to 1.5 times base, of alatae 1.75 to 2.00 times base, (fig. 16).................... Carolinaia caricis Wilson

P.t. of aptera 2 to 2.25 times base, of alatae 2.5 to 3.0 times base, (fig. 17) . . . . . . . . . . . Carolinaia cyperi Ainslie

47(45) P.t. approximately 2 times base of a.s. VI (fig. 45)

P.t. 3 or more times base of a.s. VI. . Rhopalosiphum maidis (Fitch)
VI................ 48

48(47) Siphunculi strongly swollen on distal half and usually 0.3 or longer, (fig. 46)............. Rhopalosiphum nymphaeae (L.)

Siphunculi not strongly swollen on distal half, usually less than $0.3 . \ldots \ldots \ldots \ldots \ldots \ldots \ldots \ldots \ldots \ldots \ldots \ldots \ldots . . . .49$ 
49(48) Antennae 5-segmented; setae on a.s. III distinctly longer than diameter of segment (fig. 48)

Rhopalosiphum rufiabdominalis (Sasaki)

Antennae 6-segmented; setae on a.s. III shorter than diameter of segment (fig. 47).............. Rhopalosiphum padi (L.)

50(44) Cauda with 8 or more setae................... 51

Cauda with not more than 7 setae................ 52

51(50) Abdomen bright green or greenish-yellow; R IV $+V$, and base of a.s. VI about equal (fig. 8)..........Aphis spiraecola Patch

Abdomen bright yellow; $R$ IV $+V$ approximately 1.5 times base of a.s. VI (fig. 7)..........Aphis nerii Boyer de Fonscolombe $52(50) \quad$ A.s. III of aptera pale. ...................... 53

A.s. III of aptera dark (basal $1 / 2$ may be pale)......... 56

53(52) Cubitus vestigial or absent in hindwing; length of p.t. 1.5 to 2 times length of a.s. III in alate; cauda, alate and aptera, bearing 4 setae (fig. 27)...............Hysteroneura setariae (Thomas)

Cubitus present in hindwing; p.t. and a.s. III approximately equal in length in alate; cauda, alate and aptera, usually bearing

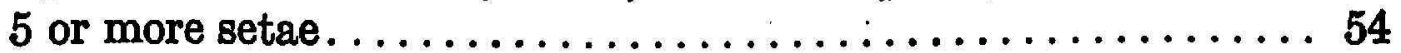

54(53) Abdomen of living material pale yellowish to dark olive green or brown; dorsum of aptera without sclerites........ 55

Abdomen of living material black; dorsum of abdomen with sclerites, sclerites may be coalesced forming a dark patch on aptera (fig. 4).....................Aphis craccivora Koch 55(54) Aptera with postsiphuncular sclerite (fig. 6) Aphis illinoisensis Shimer Aptera without postsiphuncular sclerite (fig. 5) Aphis gossypii Glover

56(52) Vertex of aptera with rectangular process; cauda usually with 4 or 6 setae........................... 57

Vertex of aptera without rectangular process; cauda usually with 7 setae (fig. 6)..............Aphis illinoisensis Shimer 57(56) Cauda usually with 6 setae; abdomen reticulated (fig. 3) Cauda usually with 4 setae (fig. 49) Aphis coreopsidis (Thomas) Schizaphis graminum (Rondani)

\section{FIELD KEY TO APTEROUS APHIDIDAE OF PUERTO RICO'}

On aerial portion of plants. Not on roots........... 4

On roots of plants.......................... 2

${ }^{1}$ Characters refer to mature aptera unless indicated otherwise. 
2 (1) On roots of Ipomea; white to yellowish with tufts of wax on sides of abdomen (fig. 24)........ Geopemphigus floccosus (Moreira)

Not on roots of Ipomea .................... 3

3 (2) On roots of grasses (fig. 51,52 )

Tetraneura nigriabdominalis (Sasaki)

Not on roots of grasses, polyphagous, white with tufts of wax on abdomen, (fig. 43)......Prociphilus erigeronensis (Thomas)

4 (1) General appearance of an aphid; not flocculent waxy or with tufts of wax on abdomen...................... 7

General appearance of a scale; or, body flocculent waxy, or with tufts of wax on abdomen..................... 5

5 (4) General appearance of a scale insect; body not flocculent

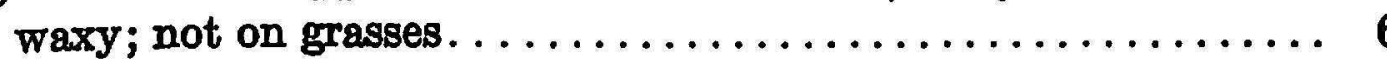

Body dark brown, powdery to flocculent waxy; head 2 hornlike projections on vertex; dark grey, powdery, with tufts of wax along lateral margins and 2 medial on abdomen, especially on caudal 2/3; nymphs light brown; on grasses (fig. 38)

Oregma panicola Takahashi

6 (5) On palms; black, round, white fringe around body; numphs $\tan$ (fig. 19) . . . . . . . . . . . . . Cerataphis variabilis H.R.L. On orchids; black, round, white fringe around body (fig. 18) Cerataphis orchidearum (Westwood)

7 (4) Antennal tubercles well developed, distinctly exceeding vertex of head, may be diverging or converging.......... 32

Antennal tubercles not well developed, not exceeding vertex of head, may be absent.......................... 8

8 (7) Not on Thuja.......................... 9

On Thuja; aptera brown, siphunculi mammiform, black (fig. 20)..................... Cinara tujafilina (Del Guercio)

9 (8) Not on Lagerstroemia..................... 10

On Lagerstroemia; nymphs yellow; setae on abdomen of nymphs spinelike, wings with dark areas at tip of veins (fig. 34) Melanocallis kahawakuokalani (Kirkaldy)

10 (9) Not on Cyperaceae......................... 14

On Cyperaceae............................. 11

11(10) Body tan to brownish-black................... 12

Body greenish with reddish tinge around and between siphunculi. On grasses, bamboo, and Cyperus (fig. 47)

12(11) Brownish-black; on Cyperus rotundus (fig. 17) Rhopalosiphum padi (L.)

Carolinaia cyperi Ainslie Tan, or brownish with white cauda; not on Cyperus rotundus. 13 
13(12) Tan, cauda not conspicuous; siphunculi constricted toward tip (fig. 16) . . . . . . . . . . . . . . Carolinaia caricis Wilson Brownish to reddish-brown; cauda white, conspicuous; siphunculi dark, not constricted toward tip (fig. 27)

Hysteroneura setariae (Thomas)

14(10) Aptera black or brown...................... 15

Aptera not black or brown, may be varying shades of white, yellow, green or grey; may be powdery . . . . . . . . . . 20

15(14) Aptera black. . . . . . . . . . . . . . . . . . . . . 16

Aptera brown or olive brown................ 17

16(15) Nymphs tan (fig. 53)

Toxoptera aurantiae (Boyer de Fonscolombe)

Nymphs greyish, pulverulent (fig. 4) ....Aphis craccivora Koch

17(15) Body uniform color; not reddish-brown around and between siphunculi............................... 18

Body not uniform color; reddish-brown around and between siphunculi; on grasses and bamboo (fig. 47). Rhopalosiphum padi (L.) 18(17) Reddish-brown; nymphs tan................... 19

Olive or greenish-brown; siphunculi swollen, may be lighter at base (fig. 46)..............Rhopalosiphum nymphaeae (L.) 19(18) Brownish; cauda white; siphunculi dark; on grasses (fig. 27) Hysteroneura setariae (Thomas)

Reddish-brown to black; on Cissus and Vitis; antennae dark only at joints and beyond basal portion of a.s. VI; cauda dark; siphunculi dark; not on grasses (fig. 6)...Aphis illinoisensis Shimer 20(14) Body without spine-like setae................. 21

Body with spine-like setae; bright lemon-yellow, dorsum of abdomen with rows of dark spots (fig. 50).... Sipha flava (Forbes) 21(20) Not on crucifers.......................... 23

On crucifers............................. 22

22(21) Pale green, very powdery on body; siphunculi small, swollen (fig. 12)........................ Brevicoryne brassicae (L.)

Yellowish-green, very little powder on body; siphunculi longer and not as distinctly swollen as on $B$. brassicae (fig. 28)

Lipaphis pseudobrassicae (Davis) 23(21) Aptera green to bright green or yellow, uniform color..... 28 Aptera bluish-green, white, pale yellowish-white or yellowgreen; if bluish-green, green or yellow-green, not uniform color. . . 24 24(23) Aptera white, pale yellow or yellowish-green, not darker green to black or reddish around siphunculi............. 27

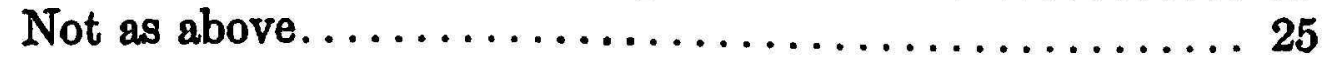


25(24) Head, thorax, and part of abdomen greenish; caudal 1/2 of abdomen reddish-brown (fig. 48)

Rhopalosiphum rufiabdominalis (Sasaki)

Not as above........................... 26

26(25) Body bluish-grey-green, darker green to black around siphunculi; body distinctly elongate (fig. 45)

Rhopalosiphum maidis (Fitch)

Body dull green, reddish tinge between siphunculi (fig. 47)

Rhopalosiphum padi (L.)

27(24) Bright yellow or bright green, or dark mottled green due to embryos showing through integument; antennae dark beyond basal $1 / 2$ or a.s. III; head with rectangular process; cauda black (fig. 3)....................Aphis coreopsidis (Thomas)

Pale white, pale yellow, yellowish-green or dark mottled green; a.s. III-V pale; head without rectangular process; cauda pale; alatoid nymphs greyish with white flecks on either side of abdomen..................... Aphis gossypii Glover

28(23) Mottled greenish-yellow (bright yellow on Clibadium; green on Bidens; head may be yellowish to orange); antennae dark beyond basal 0.5 of a.s. III; head with rectangular process on vertex; cauda and siphunculi black, without postsiphuncular sclerite (fig. 3).....................Aphis coreopsidis (Thomas)

Uniformly green or yellow; head without rectangular process on vertex. .............................. 29

29(28) Not on Gynura; greenish or bright yellow; cauda conspicuous; siphunculi imbricated........................ 30

On Gynura; yellowish; cauda inconspicuous; siphunculi smooth, not imbricated (fig. 11) ... Brachycaudus helichrysi (Kalt.)

30(29) Aptera and nymphs bright yellow; on Asclepias, Calotropis, and Nerium (fig. 7)..........Aphis nerii Boyer de Fonscolombe Aptera and nymphs greenish. . . . . . . . . . . . . 31

31(30) Aptera bright green; siphunculi and cauda black; polyphagous (fig. 8).................... Aphis spiraecola Patch Aptera pale green; siphunculi dark on distal 0.3 only (fig. 49)

Schizaphis graminum (Rondani)

32 (7) Body of uniform color, may be slightly darker around siphunculi.

Abdomen with a U-shaped area, a dark spot, or a dark stripe down the middle of the abdomen, or, head and tip of abdomen (cauda) yellowish-orange to reddish-brown and contrasting with rest of body $. . . \ldots \ldots \ldots \ldots \ldots \ldots \ldots \ldots \ldots \ldots \ldots . \ldots \ldots$ 
33(32) Abdomen some shade of reddish-brown to black........ 34 Abdomen some shade of white, yellow, tan or green...... 37

34(33) On Compositae; body reddish-brown; wing veins not bordered with fuscous (fig. 21)........ Dactynotus ambrosiae (Thomas)

On Leguminosae or Musaceae; body reddish-brown to brownish-black: some wing veins fuscous............. 35

35(34) On Musaceae (fig. 40).........Pentalonia nigronervosa Coquerel

On Leguminosae. ........................... 36

36(35) Aptera shiny, reddish-brown; nymphs reddish-brown (fig. 42)

Picturaphis puertoricensis Smith

Aptera brownish-black to black; nymphs dark, dull brown-

ish-black to black (fig. 41)....... Picturaphis brasiliensis (Moreira)

37(33) Setae on head pointed to blunt................. 40

Setea on head knobbed to fan shape................ 38

38(37) On Fragaria; small, body less than 1.5, pale yellowish-white (fig. 15)........... Capitophorus (Pentatrichopus) minor (Forbes)

Not on Fragaria; body 1.9 to 2.5 , white or pale greenish or pale yellowish.............................. 39

39(38) On Cirsium; light green to powdery white; siphunculi reaching distinctly beyond tip of cauda (fig. 13)

Capitophorus elaeagni (Del Guercio)

On Polygonum, whitish to pale yellow; siphunculi not reaching distinctly beyond tip of cauda (fig. 14)

Capitophosus hippophae subsp. javanicus H.R.L.

40(37) Not on Mentha........................ 41

On Mentha; very small, whitish to light green; antennal tubercles strongly convergent (fig. 39). .Ovatus crataegarius (Walker)

41(40) Yellowish-green or dark green around base of siphunculi, contrasting with whitish to yellowish, or greenish abdomen.... 42

Not as above............................. 43

42(41) Whitish to pale yellow, yellowish-green around siphunculi; tip of siphunculi black (fig. 10)......... Aulacorthum solani (Kalt.)

Bright green, darker green around base of siphunculi (fig. 1) Acyrthosiphon bidenticola Smith

43(41) Not on $R o s a \ldots \ldots \ldots \ldots \ldots \ldots \ldots \ldots \ldots \ldots \ldots \ldots, 45$

On Rosa.............................. 44

44(43) Abdomen light green, head and thorax same color as abdomen; siphunculi pale (fig. 44)..... Rhodobium porosum (Sanderson) Abdomen dark green, head brownish-black; siphunculi black (fig. 31)

45(43) Antennal tubercles convergent.................. 46 Antennal tubercles not convergent............... 49 
46(45) Yellowish to $\tan , \ldots \ldots \ldots \ldots \ldots \ldots \ldots \ldots \ldots \ldots \ldots \ldots \ldots$

Whitish to yellowish-green. ................. 48

47(46) Antennae longer than body; abdomen greenish-brown to tan with reddish area around siphunculi; on Ludwigia octovalis = Jussiaea angustifolia (fig. 25)......... Hyalomyzus jussiaeae Smith

Antennae shorter than body; abdomen dirty yellowish-tan with dark dashes on sides of abdomen (fig. 36). Myzus ornatus Laing 48(46) Yellowish-green; siphunculi not swollen; antennal tubercles strongly convergent; polyphagous (fig. 37 )

Myzus persicae (Sulzer)

Light whitish-green; siphunculi swollen; antennal tubercles only slightly convergent; aptera with rectangular process on vertex of head (fig. 26)...............Hyperomyzus lactucae (L.)

49(45) Distal $1 / 2$ to $2 / 3$ of siphunculi dark, on Leptilon......... 50

Only tip of siphunculi dark................... 51

50(49) Cauda dusky to dark (alates); siphunculi subequal to a.s.

III (fig. 22)............... Dactynotus erigeronensis (Thomas)

Cauda pale (alates); siphunculi distinctly shorter than a.s.

III (fig. 23)................. Dactynotus gravicornis (Patch)

51(49) Whitish-yellow; siphunculi swollen, pale, tips dark (fig. 2)

Amphorophora commelinensis Smith

Greenish or pinkish; siphunculi not swollen.......... 52

52(51) Bright green, darker green at base of siphunculi; siphunculi dusky; on Bidens (fig. 1)..........Acyrthosiphon bidenticola Smith

Pale green or pink or whitish; tip of siphunculi dark......53

53(52) Pale green or pink; antennae dark, base of a.s. III may be pale (fig. 29)...............Macrosiphum euphorbiae (Thomas)

Pale whitish-green, appearing somewhat powdery; antennae

light, dark at joints (fig. 26)........... Hyperomyzus lactucae (L.)

54(32) Abdomen yellowish-green to bright yellow, and bearing a dark U-shaped area or a black spot................. 55

Abdomen greenish to brownish; head and cauda orange to reddish-orange.$\ldots \ldots \ldots \ldots \ldots \ldots \ldots \ldots \ldots \ldots \ldots \ldots \ldots$

55(54) Bright yellow with black spot on abdomen; siphunculi black (fig. 30)............Macrosiphum (Sitobion) luteum (Buckton)

Whitish to yellowish-green with a U-shaped dark area on abdomen; antennal tubercles slightly convergent; tip of siphunculi dark (fig. 9)..Aulacorthum (Neomyzus) circumflexum (Buckton) 56(54) Abdomen with darker medial stripe; specimens in the same colony may vary from a basic greenish to brownish color with yellowish to brownish head or cauda; not on Desmodium (fig. 32) Macrosiphum (Sitobion) salviae Bartholomew 
Abdomen without darker medial stripe; basic color greenish with yellowish to brownish head and cauda and large dark area around siphunculi; On Desmodium (fig. 35)

Microparsus olivei Smith and Tuatay

\section{ADDITIONS AND CORRECTIONS}

\section{Acyrthosiphon bidenticola Smith}

Fig. 1

On Bidens pilosa, (67-129), Barranquitas-Naranjito Rd., Km. 9.9, Apr. 7, 1967, (CFS, CKS, MEP); (67-97), Maricao-Sabana Grande Rd., Apr. 4, 1967, (CFS, CKS, LFM, RW).

\section{Amphorophora commelinensis Smith}

Fig. 2

On Callisia repens, (67-173), Barranquitas-Villalba Rd., Km. 3.5, Apr. 11, 1967, (CFS, CKS, LFM).

On Commelina coelestris, (60-74), Mt. Britton Rd., El Yunque, alt. 2,100 ft., Apr. 20, 1960, (CSF, LFM, RW); (67-218), Mt. Britton Loop Rd., El Yunque, Apr. 13, 1967, (CFS, CKS, LFM, RW).

On Commelina diffusa = Commelina longicaulis, (67-172), BarranquitasVillalba Rd., Km. 3.5, Apr. 11, 1967, (CFS, CKS, LFM); (67-36), CayeySalinas Rd., Peñon del Collao, alt. 2,000 ft., Apr. 2, 1967, (CFS, CKS, LFM); (67-21), El Yunque, Apr. 1, 1967, (CFS, CKS, MEP); (67-138), Rio Grande, Apr. 9, 1967, (CFS, CKS, MEP).

\section{Aphis coreopsidis (Thomas)}

\section{Fig. 3}

On Bidens pilosa, (67-55), Arecibo-Lares Rd., Km. 3.4, Apr. 3, 1967, (CFS, CKS, MEP, RW); (55-162 \& 55-170), El Yunque, Km. 10.5, Mar. 9, 1955, (CFS).

On Bidens reptans, (67-52), Arecibo-Lares Rd., Km. 3.4, Apr. 3, 1967, (CFS, CKS, MEP, RW).

On Clibadium erosum, (67-183), Cerro Punta-Jayuya, alt. 4,300 ft., Apr. 11, 1967, (CFS, CKS, LFM); (67-23), El Yunque, Km. 7.6, Apr. 1, 1967, (CFS, CKS, MEP); (67-205), El Yunque Peak, Apr. 13, 1967, (CFS, CKS, LFM, RW); (69-68), El Yunque, one Km. from top, Mar. 13, 1968, (CFS, LFM, RW); (67-143), La Santa, Guavate Mts., Cayey, Apr. 10, 1967, (CFS, CKS, SMG).

On Cosmos caudatus, (69-50), Adjuntas-Castañer Rd., $\mathrm{Km}$. 68.5, alt. 2,020 ft., Mar. 11, 1969, (CFS, CKS, LFM, SMG). 
On Ilex macfadyenii, (69-20), El Yunque, East Peak Rd., alt. 2,300 ft., Mar. 7, 1969, (CFS, CKS, LFM, RW).

On Mikania pachyphylla, (69-15), El Yunque, East Peak Rd., alt. 3,000 ft., Mar. 7, 1969, (CFS, CKS, LFM, RW).

On Carica papaya, (PR No. 2) Rd. 112, Km. 1.2, Oct. 19, 1967, (G. A. Schaefers).

\section{Aphis craccivora Koch}

Fig. 4

On Borreria ocimoides, (60-29), Río Piedras, Apr. 18, 1960, (CFS, LFM).

On Lablab niger $=$ Dolichos lablab, (67-116), Guayabal Lake, Villalba, Rd. No. 149, Km. 62, Apr. 6, 1967, (CFS, CKS, LFM, RW).

On Macroptilium lathyroides = Phaseolus lathyroides, (67-69), Adjuntas Agricultural Substation, Apr. 3, 1967, (CFS, CKS, MEP, RW).

On Phaseolus schotti = Phaseolus trichocarpus, (69-31), Florida, east of Arecibo, Mar. 10, 1969, (CFS, CKS, MEP).

On Portulaca oleracea, (69-26), Capetillo St. No. 4, Río Piedras, Mar. 8, 1969, (CFS, CKS, LFM); (67-125), Naranjito-Barranquitas Rd., Apr. 6, 1967, (CFS, CKS, LFM, RW).

On Tephrosia senna = Cracca cathartica, (67-114), El Tuque Beach, Ponce, Apr. 6, 1967, (CFS, LFM, RW, SMG).

On Tephrosia cinerea = Cracca cinerea, (67-113), Desecheo Island, Apr. 5, 1967, (CFS, LFM, RW); (67-115), El Tuque Beach, Ponce, Apr. 6, 1967, (SMG CFS, LFM, RW).

On Vicia faba, (69-30), Florida, east of Arecibo, Mar. 10, 1969, (CFS, CKS, MEP).

\section{Aphis gossypii Glover}

Fig. 5

On Adenostrema verbesina, (67-91), Mayagüez-Maricao Rd., Km. 21.6, Apr. 4, 1967, (CFS, CKS, LFM, RW).

On Anoda acerifolia, (67-65), Lares-Yauco Rd., Apr. 3, 1967, (CFS, CKS, MEP, RW).

On Anthocephalus cadamba, (59-8), Catalina Nursery, El Yunque Rd., Km. 4.2, Mar. 6, 1969, (CFS, CKS, LFM, RW).

On Cajanus cajan = Cajanus indicus, (67-152), Cayey-Guayama Rd., Km. 17.3, Apr. 10, 1967, (CFS, CKS, SMG).

On Cassia occidentalis = Ditremexa occidentalis, (69-75), Río Piedras, Mar. 14, 1969, (CFS, MEP, Miss J. Sánchez).

On Cassia tora = Emelista tora, (69-57), south shore of Lake Tortuguero, Vega Baja, Mar. 12, 1969, (CFS, RW). 
On Cestrum macrophyllum, (67-215), El Yunque, (The Rock), Apr. 13, 1967, (CFS, CKS, LFM, RW).

On Clerodendron fragans, (69-38), Utuado-Adjuntas Rd., Mar. 11, 1969, (CFS, LFM, SMG).

On Clibadium erosum, (67-145), La Santa, Guavate Forest, Cayey, Apr. 10, 1967, (CFS, CKS, SMG).

On Coccoloba uvifera, (67-8), Freeport, Bahamas, Mar. 29, 1967, (CFS). On Coffea robusta, (69-41), Adjuntas-Castanier Rd., Km. 77.7, alt. 1,720 ft., Mar. 11, 1969, (CFS, CKS, LFM, SMG).

On Commelina sp., (69-76), Río Piedras, Mar. 14, 1969, (CFS, MEP).

On Crossandra infundibuliformis, (67-157), Naranjito-Barranquitas Rd., Km. 13.1, Apr. 11, 1967, (CFS, CKS, LFM).

On Cucumis sativus, Cupey Alto, Río Piedras, May 13, 1968, (J. Bird, Miss J. Sánchez).

On Cyathea arborea, (67-137), Río Grande-El Verde Rd., Km. 13.9, Apr. 9, 1967, (CFS, CKS, MEP).

On Epidendrum secundum = Amphiglottis secunda, (67-194), Doña Juana, Jayuya Rd., Km. 8.9, Apr. 2, 1967, (CFS, CKS, MEP).

On Guettarda scabra, (69-62), south side of Lake Tortuguero, Vega Baja, Mar. 12, 1969, (CFS, RW).

On Hibiscus eetveldeanus, (69-24), Cerro La Marquesa, Aguas Buenas (radio and TV towers), Mar. 8, 1969, (CFS, CKS, LFM).

On Hibiscus furcellatus, (69-58), south shore, Lake Tortuguero, Mar. 12, 1969, (CFS, RW).

On Hyptis pectinata, (67-168), Barranquitas-Villabla Rd., Km. 3.5, Apr. 11, 1967, (CFS, CKS, LFM).

On Melothria guadalupensis, (67-199) Doña Juana-Jayuya Rd., Km. 8.9, Apr. 12, 1967, (CFS, CKS, MEP).

On Mikania cordifolia, (67-84), Mayagüez-Maricao Rd., Km. 11.6, Apr. 4; 1967, (CFS, CKS, LFM, RW).

On Musa paradisiaca, Corazal, Feb. 19, 1968, (Miss J. Sánchez); (69-74), Río Piedras, Mar. 14, 1969, (CFS, MEP, Miss Sánchez).

On Phaseolus lunatus, (69-34), Florida-Jayuya Rd., Km. 40.3, Mar. 10, 1969, (CFS, CKS, MEP).

On Piper peltatum = Pothomorphe peltata, (67-62), Lares-Yauco Rd., Apr. 3, 1967, (CFS, CKS, MEP, RW); (67-17), El Yunque, Apr. 1, 1967, (CFS, CKS, MEP).

On Plantago major, (67-216), El Yunque, Apr. 13, 1967, (CFS, CKS, LFM, RW).

On Saggitaria lancifolia, (69-6), Toa Baja, Mar. 5, 1969, (CFS, MEP, RW).

On Salvia occidentalis, (67-160), Barranquitas-Villalba Rd., Km. 3.5, 
Apr. 11, 1967, (CFS, CKS, LFM); (67-63), Lares-Yauco Rd., Km. 6.7, Apr. 3, 1967, (CFS, CKS, MEP, RW).

On Sanchezia nobilis, (67-79), Mayaguez-Maricao Rd., Km. 11.6, Apr. 4, 1967, (CFS, CKS, LFM, RW).

On Sida acuta = Sida carpinifolia, (67-64), Lares-Yauco Rd., Apr. 5, 1967, (CFS, CKS, MEP, RW).

On Sida rhombifolia, (67-219), Mt. Britton Loop Rd., El Yunque, Apr. 13, 1967, (CFS, CKS, LFM, RW).

On Tournefortia bicolor, (69-64), south side of Lake Tortuguero, Vega Baja, Mar. 12, 1969, (CFS, RW).

On Triplaris cumingiana, (69-77), Río Piedras, Mar. 14, 1969, (CFS, MEP).

On Turnera ulmifolia, (69-56), south shore of Lake Tortuguero, Vega Baja, Mar. 12, 1969, (CFS, RW).

On Xanthosoma nigrum = Xanthosoma violaceum, (67-46), Arecibo, Apr. 3, 1967, (CFS, CKS, MEP, RW).

\section{Aphis illinoisensis Shimer}

Fig. 6

On Cissus sicyoides, (67-85), Mayaguez-Maricao Rd., Km. 21.4, Apr. 4, 1967, (CFS, CKS, LFM, RW).

\section{Aphis nerii Boyer de Fonscolombe}

Fig. 7

On Asclepias curassavica, (67-40), Peñón del Collao, Cayey-Aibonito Rd., alt. 2,000 ft., Apr. 2, 1967, (CFS, CKS, LFM); (69-16), East Peak Rd., El Yunque, alt. 2,300 ft., Mar. 7, 1969, (CFS, CKS, LFM, RW).

On Asclepias nivea, (67-60), Arecibo-Lares Rd., Km. 34, Apr. 3, 1967, (CFS, CKS, MEP, RW); (69-47), Guilarte Forest, Adjuntas Rd., Km. 7.2, Mar. 11, 1969, (CFS, CKS, LFM, SMG).

On Carica papaya, (PR No. 3), Rd. No. 181, Oct. 25, 1967, (Schaefers). On Nerium oleander, (67-11), Freeport, Bahamas, Mar. 21, 1967, (CFS).

\section{Aphis spiraecola Patch}

Fig. 8

On Anthocephalus cadamba, (69-8), Catalina Nursery, El Yunque Rd., Km. 4.2, Mar. 6, 1969, (CFS, CKS, LFM, RW).

On Carica papaya, (PR No. 4), Fortuna Substation, Ponce, Jan. 3, 1968, (G. A. Schaefers); (PR No. 1), Isabela Substation, Oct. 19, 1967, (G. A. Schaefers); (PR No. 7), Isabela, Feb. 15, 1968, (G. A. Schaefers); (PR 
No. 6), Mayaguez, Feb. 15, 1968, (G. A. Schaefers); (PR No. 5), Río Piedras, Agricultural Experiment Station, Jan. 10, 1968, (G. A. Schaefers).

On Celosia sp., (67-74), Yauco-Lares Rd., at Castañer, Apr. 3, 1967, (CFS, CKS, MEP, RW).

On Cecropia peltata, (67-140), Río Grande-El Verde Rd., Km. 13.9, Apr. 9, 1967, (CFS, CKS, MEP).

On Cestrum laurifolium, (69-54), south shore of Lake Tortuguero, Vega Baja, Mar. 12, 1969, (CFS, RW).

On Cestrum macrophyllum, (67-214), El Yunque, Apr. 13, 1967, (CFS, CKS, LFM, RW).

On Chrysanthemum sp., Río Piedras, Mar. 27, 1968, (J. Bird, Miss Josefina Sánchez).'

On Cissampelos pareira, (69-9), El Yunque Rd., $\mathrm{Km}$. 8.8, Mar. 6, 1969, (CFS, CKS, LFM, RW).

On Citrus sinensis, (67-15), El Yunque Rd., Km. 2.4, Apr. 1, 1967, (CFS, CKS, MEP).

On Citrus sp., Maricao, Apr. 27, 1968.

On Clibadium erosum, (69-67), El Yunque, one kilometer from El Yunque Peak, Mar. 13, 1969, (CFS, RW, LFM).

On Dieffenbachia sequine, (67-81), Mayagüez-Maricao Rd., Km. 11.6, Apr. 4, 1967, (CFS, CKS, LFM, RW).

On Ditomitis montana = Octadesmia montana, (69-71), El Yunque, one mile from El Yunque Peak, Mar. 13, 1969, (CFS, LFM, RW).

On Epidendrum prismatocarpum, (67-224), Pennock Nursery, Río Piedras, Apr. 15, 1967, (CFS, LFM).

On Epidendrum secundum = Amphiglottis secunda, (67-223), Pennock Nursery, Río Piedras, Apr. 15, 1967, (CFS, LFM).

On Eupatorium dolicholepis, (67-56), Arecibo-Lares Rd., Km. 34, Apr. 3, 1967, (CFS, CKS, MEP, RW).

On Ipomoea batatas, (67-16), El Yunque, Apr. 1, 1967, (CFS, CKS, MEP).

On Ixora coccinea, Río Piedras, Mar. 27, 1968, (Bird, Sánchez).

On Nothopanax quilfoylei = Polyscias quilfoylei (67-222), El Yunque, Apr. 13, 1967, (CFS, CKS, LFM, RW).

On orange orchid, (67-226), Pennock Nursery, Río Piedras, Apr. 15, 1967, (CFS, LFM).

On Osmanthus (?), (67-10), Freeport, Bahamas, Mar. 30, 1967, (CFS). On Piper aduncum, (67-18), El Yunque, Apr. 1, 1967, (CFS, CKS, MEP).

On Piper peltatum = Pothomorphe peltata, (67-17), El Yunque, Apr. 1, 1967, (CFS, CKS, MEP).

On Plumiera obtusa = Plumiera krugii, (67-104), Maricao-Sabana Grande Rd., Apr. 4, 1967, (CFS, CKS, LFM, RW). 
On Rosa, sp., (67-68), Adjuntas Agricultural Substation, Apr. 3, 1967, (CFS, CKS, MEP, RW).

On Salix chilensis, (67-130), Barranquitas-Naranjito Rd., Km. 11.3, Apr. 7, 1967, (CFS, CKS, MEP).

On Sonchus oleraceus, (67-41), Barrio Sonadora, Aguas Buenas, 1,300 ft. alt., Apr. 2, 1967, (CFS, CKS, LFM).

On Trema micrantha, (67-182), Cerro de Punta, Jayuya, (Toro Negro Forest), 4,000 ft. alt., Apr. 6, 1967, (CFS, SMG, LFM, RW).

On Urena lobata, (67-122), Cerro de Punta area, Jayuya (Toro Negro Forest), 4,000 ft. alt., Apr. 6, 1967, (CFS, SMG, LFM, RW).

On walking iris, (67-96), Fish Hatchery, Maricao, Apr. 4, 1967, (CFS, CKS, LFM, RW).

\section{Aulacorthum (Neomyəus) circumflexum (Buckton)}

Fig. 9

On Commelina coelestris, (60-74), Mt. Britton Rd., El Yunque area, 2,100 ft. alt., Apr. 20, 1960, (CFS, LFM, RW).

On Dilomilis montana = Octadesmia montana, (69-12), East Peak Rd., El Yunque area, 2,600 ft. alt., Mar. 7, 1969, (CFS, CKS, LFM, RW).

On Epidendrum secundum = Amphiglottis secunda, (67-185), CialesVillalba Rd., Km. 44.3, Apr. 11, 1967, (CFS, CKS, LFM).

On Lablab niger = Dolichos lablab, (69-42), Adjuntas-Castañer Rd., Km. 77.7, alt. 1,700 ft., Mar 11, 1969, (CFS, CKS, LFM, SMG)

On Lactuca intybacea = Brachyramphus intybaceus, (51-59), CialesVillalba Rd., Mar. 22, 1959, (LFM, SMG).

On Malvaviscus grandiflorus, (60-123; 60-124), Cayey-Guayama Rd., Km. 10.4, 1,920 ft. alt., Apr. 25, 1960, (CFS, MEP).

On Rubus rosaefolius, (67-207), El Yunque Peak, Apr. 23, 1967, (CFS, CKS, LFM, RW).

On Solanum ciliatum. (67-92), road to fish hatchery, Maricao, Apr. 4, 1967, (CFS, CKS, LFM, RW).

On Vernonia cinerea, (67-87), Mayagüez-Maricao Rd., Km. 21.4, Apr. 4, 1967, (CFS, CKS, LFM, RW).

\section{Aulacorthum solani (Kaltenbach)}

Fig. 10

On Bidens pilosa, (67-71), Rd. No. 135, 2 miles west, Castañer, Apr. 3, 1967, (CFS, CKS, MEP, RW).

On Clibadium erosum, (67-144), La Santa, Guavate Forest, Cayey, Apr. 10, 1967, (CFS, CKS, SMG). 
On Elephantopus scaber = Elephantopus mollis, (67-174), BarranquitasVillalba Rd., Km. 3.5, Apr. 11, 1967, (CFS, CKS, LFM).

On Lantana camara, (67-196), Doña Juana-Jayuya Rd., Km. 8.9, Apr. 11, 1967, (CFS, CKS, LFM).

On Rubus rosaefolius, (67-127), Barranquitas-Naranjito Rd., $\mathrm{Km} .9 .9$, Apr. 7, 1967, (CFS, CKS, MEP); (67-208), El Yunque, Apr. 13, 1967, (CFS, CKS, LFM, RW).

On scrophulariaceous plant, (67-206), El Yunque Peak, Apr. 13, 1967, (CFS, CKS, LFM, RW).

On Zantedeschia aethiopica, (67-176), Barranquitas, Apr. 11, 1967, (CKS, CFS, LFM).

\section{Capitophorus elaeagni (Del Guercio)}

Fig. 13

Principal Diagnostic Characters: Tip of siphunculi dark; abdominal segments VI-VIII with 4 to 8 knobbed setae which are distinctly longer than setae on abdominal segments I-V.

On Cirsium mexicanum, (67-153), Guayama-Cayey Rd. No. 15, Km. 17.3, Apr. 10, 1967, (CFS, CKS, SMG); (69-2), La Torre, Barrio Boquerón, Barranquitas, Mar. 5, 1969, (CFS, CKS, MEP, RW).

\section{Capitophorus hippophaes subsp. javanicus Hille Ris Lambers}

Fig. 14

Capitophorus hippophaes subsp. javanicus H. R. L. 1953:156 (5).

Apterous vivipara: Color of living specimens whitish. Cleared specimens pale on body and appendages. Measurements of one specimen. Body 1.35. Width of head 0.31. A.s. III, 0.31; IV, 0.20; V, 0.18; VI, $0.09+0.25$. R IV + V, 0.07. Hind tibia 0.71. Hind tarsomere II, 0.08. Siphunculus 0.40. Cauda 0.20. Antennae without secondary sensoria. Vertex of head with conspicuous knobbed setae 0.03 , abdominal segments VII and VIII each bear 2 setae similar to those on the vertex. Other setae on the dorsum of the abdomen and on antennal segments short, inconspicuous, blunt to slightly knobbed 0.004 . R IV $+V$ with 2 accessory setae. Cauda with 7 setae.

Principal Diagnostic Characters: Abdominal segments VII and VIII each with 2 knobbed setae similar to those on the vertex.

Types: Collection of D. Hille Ris Lambers, Bennekom.

Collections: On Persicaria densiflorum = Polygonum portoricensis, (67192), Dofia Juana-Jayuya Rd., Km. 6.6, Apr. 11, 1967, (CFS, CKS, MEP).

On Persicaria sp. = Polygonum sp., (69-51), Jayuya, (cross of roads No. 144 and 528), Mar. 11, 1969, (CFS, CKS, LFM, SMG).

New record for Puerto Rico. 


\section{Carolinaia cyperi Ainslie}

Fig. 17

On Cyperus sp., (69-7), Río Piedras, Mar. 6, 1969, (CFS, CKS).

On Cyperus polystachyos = Cyperus odoratus, (69-61), south side of Lake Tortuguero, Vega Baja, Mar. 12, 1969, (CFS, RW).

On Rhynchospora nervosa $=$ Dichromena ciliata, (69-35), Florida-Jayuya Rd., Km. 40.3, Mar. 10, 1969, (CFS, CKS, MEP).

\section{Cerataphis orchidearum (Westwood)}

Fig. 18

On Epidendrum secundum = Amphiglottis secunda, Villalba-Ciales Rd., Km. 44.6, Apr. 6, 1967, (CFS, CKS, LFM, RW); (67-197), Doña JuanaJayuya Rd., Km. 8.9, Apr. 12, 1967, (CFS, CKS, MEP); (67-186), VillalbaCiales Rd., Km. 44.3, Apr. 11, 1967, (CFS, CKS, LFM).

\section{Cerataphis variabilis Hille Ris Lambers}

Fig. 19

On Ptychosperma macarthurii, (67-141), Interior patio of agronomy building, Agricultural Experiment Station, Río Piedras, Apr. 10, 1967, (CFS, LFM); (69-27), Río Piedras, Mar. 9, 1969, (CFS, MEP).

\section{Dactynotus ambrosiae (Thomas)}

Fig. 21

On Chrysanthemum anethifolium, (67-184), Cerro Punta, Jayuya, alt. 4,400 ft., Apr. 11, 1967, (CFS, CKS, LFM).

On Clibadium erosum, (69-80), Maricao Forest, Mar. 14, 1969, (CFS, CKS, MEP).

On Elephantopus scaber = Elephantopus mollis, (67-86), MayagüezMaricao Rd., Km. 21.4, Apr. 4, 1967, (CFS, CKS, LFM, RW); (67-167), Villalba-Barranquitas Rd., Km. 3.5, Apr. 11, 1967, (CFS, CKS, LFM).

On Eupatorium odoratum = Osmia odorata, (67-43), Barrio Sonadora, Aguas Buenas Rd. No. 792, 1,300 ft. alt., Apr. 2, 1967, (CFS, CKS, LFM).

On Galinsoga ciliata, (67-88), Mayagüez, Apr. 4, 1967, (CFS, CKS, LFM, $\mathrm{RW}$.

On Gnaphalium portoricense, (67-169), Barranquitas, Apr. 11, 1967, (CFS, CKS, LFM).

On Pluchea carolinensis = Pluchea odorata, (67-53), Arecibo-Lares Rd., Km. 34, Apr. 3, 1967, (CFS, CKS, MEP, RW).

On Solanum torvum, (67-99), Maricao-Sabana Grande Rd., $\mathrm{Km}$. 18.4, Apr. 4, 1967, (CFS, CKS, LFM, RW). 
On Sonchus oleraceus, (67-42), Barrio Sonadora, Aguas Buenas Rd. No. 792, 1,300 ft. alt., Apr. 2, 1967, (CFS, CKS, LFM).

On Trema lamarckiana, (67-100), Maricao-Sabana Grande Rd., Apr. 4, 1967, (CFS, CKS, LFM, RW).

On Trema micrantha, (67-102), Maricao-Sabana Grande Rd., Km. 18.4, Apr. 4, 1967, (CFS, CKS, LFM, RW).

On Waltheria indica = Waltheria americana, (67-103), Mariaco, Apr. 4, 1967, (CFS, CKS, LFM, RW).

\section{Dactynotus erigeronensis (Thomas)}

Fig. 22

On Conyza canadensis $=$ Leptilon pusillum, (67-178), Cerro Punta, Jayuya, 4,000 ft. alt., Apr, 11, 1967, (CFS, CKS, LFM); (67-179), Jayuya, Apr. 11, 1967, (CFS, CKS, LFM).

\section{Dactynotus gravicornis (Patch)}

Fig. 23

On Conyza canadensis = Leptiton pusillum, (67-179), Cerro Punta, Jayuya, 4,000 ft. alt., Apr. 11, 1967, (CFS, CKS, LFM); (69-78), Maricao Forest, Mar. 14, 1969, (CFS, CKS, MEP).

\section{Geopemphigus floccosus (Moreira)}

Fig. 24

On Ipomea batatas, on roots, (67-14), Km. 2.4, El Yunque, Apr. 1, 1967, (CFS, CKS, MEP).

\section{Hyalomyzus jussiaea Smith}

Fig. 25

On Ludwigia octovalvis = Jussiaea angustifolia, (67-26), El Yunque, Apr. 1, 1967, (CFS, CKS, MEP); (69-17), El Yunque, East Peak Rd., 2,300 ft. alt., Mar. 7, 1969, (CFS, CKS, LFM, RW); (67-135), Orocovis, Apr. 7, 1967, (CFS, CKS, MEP); (69-40), Utuado-Adjuntas Rd., Mar. 11, 1969, (CFS, CKS, LFM, SMG).

\section{Hyperomyzus lactucae (Linnaeus)}

Fig. 26

On Cirsium mexicanum, (69-79), Maricao Forest, Mar. 14, 1969, (CFS, CKS, MEP). 
On Sonchus oleraceus, (69-4), La Torre, Barrio Boquerón, Barranquitas, Mar. 5, 1969, (CFS, MEP, RW); (67-112), Maricao-Sabana Grande Rd., Km. 13.9, Apr. 4, 1967, (CFS, CKS, LFM, RW).

\section{Hysteroneura setariae (Thomas)}

Fig. 27

On Andropogon bicornis, (67-105), Maricao-Sabana Grande Rd., $\mathrm{Km}$. 18.4, Apr. 4, 1967, (CFS, CKS, LFM, RW).

On Arundo donax, (60-83), Ponce-Santa Isabel Rd., Apr. 21, 1960, (CFS, LFM, MEP).

On Bambusa bambos = Bambusa vulgaris, (67-200), Ciales-Villalba Rd., Km. 33.1, Apr. 12, 1967, (CFS, CKS, MEP).

On Boerhaavia diffusa = Boerhaavea coccinea, (67-181), Cerro Punta, Jayuya, 4,000 ft. alt., Apr. 11, 1967, (CFS, CKS, LFM).

On Panicum laxum, (69-59), south shore of Lake Tortuguero, Vega Baja, Mar. 12, 1960, (CFS, RW).

On Panicum trichoides, Villalba-Barranquitas Rd., Km. 3.4, Apr. 11, 1967, (CFS, CKS, LFM).

On Paspalum secans, (67-136), Orocovis, Apr. 7, 1967, (CFS, CKS, MEP).

On Sporobolus poiretii = Sporobolus berteroanus, (67-45), Barrio Sonadora, Aguas Buenas Rd. No. 792, 1,300 ft. alt., Apr. 2, 1967, (CFS, CKS, LFM).

\section{Macrosiphum euphorbiae (Thomas)}

Fig. 29

On Emitia coccinea, (67-106), Maricao-Sabana Grande Rd., $\mathrm{Km}$. 17.9, Apr. 4, 1967, (CFS, CKS, LFM, RW).

On Rosa sp., (67067), Adjuntas Agricultural Substation, Apr. 3, 1967, (CFS, CKS, MEP, RW).

\section{Macrosiphum (Sitobion) luteum (Buckton)}

Fig. 30

On Ditomilis montana = Octadesmia montana, (67-213), El Yunque, Apr. 13, 1967, (CFS, CKS, LFM, RW); (69-13), El Yunque East Peak Rd. area, 2,600 ft. alt., Mar. 7, 1969, (CFS, LFM, RW); (69-70), 1 mile from El Yunque Peak, Mar. 13, 1969, (CFS, LFM, RW). 


\section{Macrosipum rosae (Linnaeus)}

Fig. 31

Apterous vivipara: Body green. Head brownish-black. Siphunculi black. Cauda pale. Nymphs pale green and slightly pulverulent. Cleared specimens dark on head, antennae, tips of femora, joint of femora and tibia, tips of tibia, all of tarsomeres, siphunculi and pre- and post-siphuncular sclerites. Remaining portions of the body pale. Measurements of one specimen. Body 2.8. Head width 0.57. A.s. III, 0.98; IV, 0.75; V, 0.61; VI 0.14 + 0.90. R IV + V, 0.15. Hind tibia 1.73. Hind tarsomere II, 0.11. Siphunculi 0.70. Cauda 0.55. A.s. III with ca. 16 sensoria. $R$. IV $+V$ with 8 accessory setae. T. ch. 3-3-3.

Principal Diagnostic Characters: Black head, antennae, and siphunculi; numerous (ca. 16-20) sensoria on a.s. III of aptera.

Collections: On Rosa, (67-67), Adjuntas Agricultural Substation, Apr. 3, 1967, (CFS, CKS, MEP, RW); (69-44), Adjuntas Agricultural Substation, Mar. 11, 1969, (CFS, CKS, LFM, SMG).

New record for Puerto Rico.

\section{Macrosiphum (Sitobion) salviae Bartholomew}

Fig. 32, 33

Macrosiphum salviae Bartholomew 1932: 720(2)

Macrosiphum mesosphaeri Tissot 1934: 17 (new synonymy) (17)

Smith, Martorell, and Pérez (12) separated Sitobion and Macrosiphum on the basis of the presence or absence of a sclerotic area on the dorsum of the abdomen. This is, however, a very variable character. A long series of specimens was collected in 1967 in which the sclerotic area varied from total absence to very distinct (figs. 32,33 ). We cannot find any morphological differences and thus believe Macrosiphum mesosphaeri Tissot to be a synonym of $M$. salviae Bartholomew.

On Clibadium erosum, (67-147), La Santa, Guavate Forest, Cayey, Apr. 10, 1967, (CFS, CKS, SMG).

On Hyptis capitata, (69-39), Utuado-Adjuntas Rd., Mar. 11, 1969, (CFS, CKS, LFM, SMG).

On Hyptis pectinata, (67-37), Peñón del Collao, Cayey-Aibonito Rd., 2,000 ft. alt., Apr. 2, 1967, (CFS, CKS, LFM); (67-90), Mayagüez, Apr. 4, 1967, (CFS, CKS, LFM, RW); (67-164 \& 67-165), Naranjito-Barranquitas Rd., Km. 13.1, Apr. 11, 1967, (CFS, CKS, LFM).

On Hyptis spicigera = Hyptis americana, (67-51), Arecibo-Lares Rd., 
Km. 3.4, Apr. 3, 1967, (CFS, CKS, MEP, RW); (67-73), Rd. No. 135, 2 miles west Castañer, Apr. 3, 1967, (CFS, CKS, MEP, RW); (67-66), LaresYauco Rd., Km. 57.2, Apr. 3, 1967, (CFS, CKS, MEP, RW); (67-89), Maricao-Mayagüez. Rd. No. 105, Km. 21.4, Apr. 4, 1967, (CFS, CKS, LFM, RW).

On Inga vera = Inga inga, (67-196), Jayuya, Apr. 12, 1967, (CFS, CKS, MEP).

On Lantana camara, (67-196), Jayuya, Apr. 12, 1967, (CFS, CKS, MEP).

On Lomoplis ceratonia = Mimosa ceratonia, (67-38), El Collao, CayeyAibonito Rd., Apr. 2, 1967, (CFS, CKS, LFM); (67-98), Maricao-Sabana Grande Rd., Km. 18.4, Apr. 4, 1967, (CFS, CKS, LFM, RW).

On Salvia coccinea, (67-193), Doña Juana-Jayuya, Rd. No. 144, Apr. 12, 1967, (CFS, CKS, MEP); (67-195), Doña Juana-Jayuya Rd., Km. 8.9, Apr. 12, 1967, (CFS, CKS, LFM); (67-159), Barranquitas, Apr. 11, 1967, (CFS, CKS, LFM).

On Salvia occidentalis, (67-75), Rd. No. 135, 2 miles west of Castanier, Apr. 3, 1967, (CFS, CKS, MEP, RW); (67-61), Lares-Yauco, Rd. No. 128, Km. 6.7, Apr. 3, 1967, (CFS, CKS, MEP, RW); (67-161 \& 67-166), Barranquitas-Villalba Rd., Km. 3.5, Apr. 11, 1967, (CFS, CKS, LFM); (67126), Villalba-Ciales Rd., Km. 44.6, Apr. 6, 1967, (CFS, CKS, LFM, RW).

On Salvia splendens, (67-154), Naranjito-Barranquitas Rd., $\mathrm{Km}$. 13.1, Apr. 11, 1967, (CFS, CKS, LFM).

On Trema lamarckiana, (67-101), Maricao-Sabana Grande Rd., $\mathrm{Km}$. 18.4, Apr. 4, 1967, (CFS, CKS, LFM, RW).

\section{Melanocallis kahawaluokalani (Kirdaldy)}

Fig. 34

On Lagerstroemia indica, (69-25), Cerro La Marquesa, Aguas Buenas, 1,500 ft. alt. (WKAQ radio and TV towers), Mar. 8, 1969, (CFS, CKS, LFM); (69-72), Río Piedras, Mar. 13, 1969, (CKS).

\section{Mysus ornatus Laing}

\section{Fig. 36}

Borner (3) indicated $M$. portulacae Macch. 1883 may be an earlier name for this aphid. We have not been able to verify this.

On Ageratum conyzoides, (67-203), El Yunque Peak, El Yunque, Apr. 13, 1967, (CFS, CKS, LFM, RW).

On Bidens pilosa, (67-123), Cerro Punta area, Jayuya, Toro Negro Forest, 4,000 ft. alt., Apr. 6, 1967, (CFS, CKS, LFM, RW). 
On Celosia nitida, (67-180), Cerro Punta, Jayuya, 4,000 ft. alt., Apr. 11, 1967, (CFS, CKS, LFM).

On Clibadium erosum, (67-183), Cerro Punta, Jayuya, 4,300 ft. alt., Apr. 11, 1967, (CFS, CKS, LFM); (67-146), La Santa, Guavate Forest, Cayey, Apr. 10, 1967, (CFS, CKS, SMG).

On Coleus blumei, (67-177), Barranquitas-Villalba Rd., Km. 3.5, Apr. 11, 1967, (CFS, CKS, LFM).

On Galinsoga citiata, (67-204), El Yunque Peak, 3,500 ft. alt., Apr. 13, 1967, (CFS, CKS, LFM, RW).

On Ludwigia octovalvis = Jussiaea angustifolia, (67-135), Orocovis, Apr. 7, 1967, (CFS, CKS, MEP).

On Mentha nemorosa, (67-202), El Yunque Peak, Apr. 13, 1967, (CFS, CKS, LFM, RW).

On Ocimum basiticum, (67-133), Barranquitas-Naranjito Rd., Km. 9.9, Apr. 7, 1967, (CFS, CKS, MEP).

On Salix chilensis, (67-131), Barranquitas-Naranjito Rd., Km. 9.9, Apr. 7, 1967, (CFS, CKS, MEP).

On Salvia splendens, (67-191), Florida, Jayuya, Rd. No. 140, Apr. 12, 1967, (CFS, CKS, MEP).

On Spathodea campanulata, (67-72), Rd. No. 135, 2 miles west, Castañer, Apr. 3, 1967, (CFS, CKS, MEP, RW).

\section{Myzus (Nectarosiphon) persicae (Sulzer)}

Fig. 37

On Bidens pilosa, (67-71), Rd. No. 135, 2 miles west, Castañer, Apr. 3, 1967, (CFS, CKS, MEP, RW).

On Celosia nitida (?), (67-162), Barranquitas-Villalba Rd., Km. 3.5, Apr. 11, 1967, (CFS, CKS, LFM).

On Emitia coccinea, (67-106), Maricao-Sabana Grande Rd., Km. 17.9, Apr. 4, 1967, (CFS, CKS, LFM, RW).

On Lepidium virginicum, (69-46), Adjuntas Substation, Mar. 11, 1969, (CFS, CKS, LFM, SMG).

On Osmanthus (?), (67-10), Freeport, Bahamas, Mar. 30, 1967, (CFS). On Solanum seaforthianum, (67-156), Naranjito-Barranquitas Rd., Apr. 11, 1967, (CFS, CKS, LFM).

On Sonchus oleraceus, (67-41), Barrio Sonadora, Aguas Buenas, Rd. No. 792, 1,300 ft. alt., Apr. 2, 1967, (CFS, CKS, LFM).

On a solanaceous vine (?), (67-9), Freeport, Bahamas, Mar. 29, 1967, (CFS).

On Tournefortia bicolor, (69-63), south side of Lake Tortuguero, Vega Baja, Mar. 12, 1969, (CFS, RW). 


\section{Oregma panicola Takahashi}

Fig. 38

On Commelina diffusa = Commelina longicaulis, (67-22), El Yunque, Km. 7.6, Apr. 1, 1967, (CFS, CKS, MEP).

On Ichnanthus pallens, (67-32), El Yunque, Km. 11.3, Apr. 1, 1967, (CFS, CKS, MEP); (69-69), one kilometer from El Yunque Peak, Mar. 13, 1969, (CFS, LFM, RW); (67-139), Río Grande, Km. 13.9, Apr. 9, 1967, (CFS, CKS, MEP).

On Isachne agustifolia, (69-11), East Peak Rd., El Yunque, alt. 2,600 ft., Mar. 7, 1969, (CFS, CKS, LFM, RW).

\section{Ovatus crataegarius (Walker)}

Fig. 39

On Mentha crispa, (67-132), Barranquitas-Naranjito Rd., Km. 9.9, Apr. 7, 1967, (CFS, CKS, MEP).

\section{Pentalonia nigronervosa Coquerel}

Fig. 40

On Citrus sp., (Visitor), Lajas, Apr. 27, 1968.

On Costus cylindricus, (67-221), El Yunque, La Catalina area, Apr. 13, 1967, (CFS, CKS, LFM, RW).

On Hedychium coronarium, (67-220), El Yunque, Apr. 13, 1967, (CFS, CKS, LFM, RW).

On Musa paradisiaca, (67-171), Barranquitas-Villalba Rd., Km. 3.5, Apr. 11, 1967, (CFS, CKS, LFM); (69-33), Florida-Jayuya Rd. 140, Km. 41.3, Mar. 3, 1969, (CFS, CKS, MEP).

On Musa sapientum, (67-13), El Yunque, Km. 2.4, Apr. 1, 1967, (CFS, CKS, MEP); (69-73), Río Piedras, Mar. 14, 1969, (CKS).

On Xanthosoma caracu, (67-170), Barranquitas-Villalba Rd., Km. 3.5, Apr. 11, 1967, (CFS, CKS, LFM).

\section{Picturaphis brasiliensis (Moreira)}

Fig. 41

On Lablab niger = Dolichos lablab, (69-43), Adjuntas-Castañer, $\mathrm{Km}$. 77.7, 1,700 ft. alt., Mar. 11, 1969, (CFS, CKS, LFM, SMG); (67-189), Barceloneta-Florida Rd., Km. 62.9, Apr. 11, 1967, (CFS, CKS, MEP); (67-149), Guayama-Cayey Rd., Km. 6.5, Apr. 10, 1967, (CFS, CKS, SMG).

On Phaseolus lunatus, (67-190), Florida-Jayuya Rd., Km. 49.8, Apr. 11, 1967, (CFS, CKS, MEP); (69-29), Florida, Mar. 10, 1969, (CFS, CKS, 
MEP); (69-37), Arecibo-Utuado Rd., Km. 77.8, Mar. 11, 1969, (CFS, CKS, LFM, SMG).

On Phaseolus schottii = Phaseolus trichocarpus, (69-31 \& 69-32), Florida, west of Arecibo, Mar. 10, 1969, (CFS, CKS, MEP).

\section{Picturaphis puertoricensis Smith}

Fig. 42

Picturaphis puertoricensis Smith 1970: (13)

On Phaseolus adenanthus, El Yunque, (69-65), Mar. 13, 1969, (CFS, LFM, RW); (69-21), same location as (69-65), Mar. 7, 1969, (CFS, CKS, LFM, RW).

New record for Puerto Rico.

\section{Prociphilus erigeronensis (Thomas)}

Fig. 43

Tychea erigeronensis Thomas 1879: 168 (16)

Prociphilus erigeronensis Hottes and Frison 1931: 371 (6)

Prociphilus erigeronensis: Wolcott 1936: 118 (18)

Apterous Vivipara : Color of living specimens whitish with waxlike filaments, especially on the abdomen, which tend to make curls toward the head. Cleared specimens pale; antennae, legs, cauda, and anal plate dusky. Antennae 5 segmented. Length of body 1.7-2.4, A.s. III, 0.10-0.18, IV, 0.06-0.09; V, 0.09-0.11+0.03-0.04. R IV + V, 0.11-0.15 and bearing 4-8 accessory setae. Hind tibia, $0.36-0.47$; hind tarsus, $0.13-0.16$; t.ch. 2-2-2 or 3-2-2. Cauda rounded and bearing 12-22 setae.

Principal Diagnostic Characters: Waxy filaments which curl forward on living specimens, conspicuous wax plates on sides of abdomen, especially segment VII.

Type: Probable cotypes (slide no. 2769) in the collection of the Illinois Natural History Surveys, Hottes and Frison. (6)

Collections: Aptera on the roots of several species of plants. Only one specimen has been seen from Puerto Rico. This was intercepted by USDA Plant Quarantine personnel on "lima bean leaves, October 11, 1935, Cidra, P. R., San Juan No. 6214, McGibbin and Mills". This is an apterous specimen, therefore, it must have gotten on the leaf during transit from Cidra to the Plant Quarantine Station at San Juan. Wolcott (18) recorded Prociphilus erigeronensis (Det. W. P. Mason) resting on dahlia leaf at Guaynabo (No. 3355). We have not been able to locate this slide; there is no way of knowing whether it is apterous or alate.

New record for Puerto Rico.

1 Measurements and description from specimens collected in North Carolina. 


\section{Rhodobium porosum (Sanderson)}

Fig. 44

On Rosa, (67-67), Adjuntas Agricultural Substation, Apr. 3, 1967, (CFS, CKS, MEP, RW); (69-45), Adjuntas Agricultural Substation, Mar. 11, 1969, (CFS, CKS, LFM, SMG); (69-5), Barranquitas, Mar. 5, 1969, (Cl'S, MEP, RW); (67-151), Guayama-Cayey Rd., Km. 17.3, Apr. 10, 1967, (Cl'S, CKS, SMG), (67-155), Naranjito-Barranquitas Rd., Km. 13.1, Apr. 11, 1967, (CFS, CKS, LFM); Saint Just, Trujillo Alto, Mar. 15, 1968, (G. Rivera Mercado).

\section{Rhopalosiphum maidis (Fitch)}

Fig. 45

On Coix lachryma-jobi, (67-134), Orocovis, Apr. 7, 1967, (CFS, CKS, MEP).

On Cyperus odoratus = Cyperus ferax, (67-28), El Yunque, Apr. 1, 1967, (CFS, CKS, MEP).

On Eriochloa punctata, (67-29), El Yunque, Apr. 1, 1967, (CFS, CKS, MEP).

\section{Rhopalosiphum nymphaeae (Linnaeus)}

Fig. 46

On Dieffenbachia sequine, (67-80), Mayagüez-Maricao, Rd. No. 10ミ), lim. 11.6, Apr. 4, 1967, (Clis, CKS, LFM, RW).

\section{Rhopalosiphum padi (Linnaeus)}

lï. 47

Aphis parli Linnacus 1758: 451 (i)

Apterous Vivipara: Color of living specimens brownish. Nymphs reddish between siphunculi. Cleared specimens dusky on head, antennae, legs, siphunculi, cauda, and anal plate. Measurements of one specimen. Body 1.38. Width of head 0.37 . A.s. III, 0.20 ; IV, $0.11 ; \mathrm{V}, 0.13$; VI, $0.08+0.33$. R IV $+V, 0.10$. Hind tibia 0.63. Hind tarsomere II, 0.09. Siphunculi 0.20. Cauda 0.13. Antennae without secondary sensoriat. R IV + V with 2 accessory setae. Cauda with 4 setae. T. ch. variable, usually 3-2-2.

Collections: On Bambusa bambos = Bambusa mulgaris, (55-511) lil Yunque, 3,500 ft. alt., (MLP, LFM); (67-201), Villalba-Ciales Rd., Km. S.9, Apr. 12, 1967, (CFS, CKS, MISP).

On ('yperus rotumlus, (67-24), lil Yunque, Kim. 7.6, Apr. 1, 1967, (CKS, ('IS, Mlil). 
On Eriochloa punctala, (67-29 \& 67-30) El Yunque, Km. 7.7, Apr. 1, 1967, (CFS, CKS, MEP); (67-210), El Yunque Peak, Apr. 13, 1967, (CFS, CTSS, LFM, RW).

On Hypoxis decumbens, (67-94), road to fish hatchery, Maricao, Apr. 4, 1967, (CKS).

On Iresine diffusa = Iresine celosia, (67-1S1), Cerro Punta, Jayuya, 4,000 ft. alt., Apr. 11, 1967, (CFS, CKS, Ll AI).

On Paspalum conjugatum, (67-209), El Yunque Peak, Apr. 13, 1967, (CFS, CKS, LFM, RW).

On Passiflora rubra, (55-106), El Yunque, 3,500 ft. alt., Mar. 4, 1955 (CFS).

On Pennisetum purpureum, (69-10) East Peak Rd., El Yunque Area, 2,600 ft. alt., Mar. 7, 1969, (CFS, CKS, LF.MI, RW).

On Rhynchospora sp. (55-103), El Yunque, 3,500 ft. alt., Mar. 4, 1955, (CFS).

New record for Puerto Rico.

\section{Rhopalosiphum rufiabdominalis (Sasaki)}

lig. 48

Toxoptera rufiablominalis Sasaki 1899: 202 (11)

Siphocoryne splendens Theobald 1915: 116 (15)

Cerosipha subterranea Mason 1937: 166 (10)

Rhopalosiphum subterraneum: Wolcott 1941: 15:2 (19)

Rhopalosiphum rufiabdominalis: Eastop 1966: 496 (Synonomy) (4)

Apterous Vivipara: Color of living specimens greenish on head, reddishbrown on caudal $1 / 2$ of abdomen. Cleared specimens dusky on head, antennae, legs, siphunculi, cauda, and anal plate. Measurements of one specimen. Body 1.45. Width of head 0.38. A.s. III, 0.20; IV, 0.08; V, $0.05+$ 0.35. R IV $+V, 0.13$. Hind tibia 0.60. Hind tarsomere II, 0.09. Siphunculus 0.20 . Antennae 5 or 6 segmented, without secondary sensoria. Setae on a.s. III and body 0.07 . R IV $+V$ with 2 accessory setae. Cauda with 4 setae.

Principal Diagnostic Characters: Reddish color on caudal 1/2 of abdomen of living aptera; long setae on antennae and long processus terminalis.

Collections: On Axonopus compressus, (69-66), one km. from El Yunque Peak, El Yunque, Mar. 13, 1969, (CFS, Ll MI, RW).

New record for Puerto Rico.

\section{Sipha flava (Forbes)}

Fig. 50

On Eriochloa punctat, (67-27 it 67-31), El Yuncjue, Apr. 1, 1967, (CFS, CKS, MEP); (67-210), El Yunque, Apr. 13, 1967, (ClS, CKS, LFM, RW). 
On Panicum fasciculatum, (67-217), El Yunque, Apr. 13, 1967, (CFS, CKS, LFM, RW).

On Paspalum conjugalum, (67-209), El Yunque, Apr. 13, 1967, (CFS, CKS, LFM, RW).

On Pennisetum purpureum, (69-10), East Peak Rd., El Yunque area, 2,600 ft. alt., Mar. 7, 1969, (CFS, CliS, LFM, RW).

\section{Tetraneura nigriabdominalis (Sasaki)}

Fig. 51,52

Schizoneura nigriabdominalis Sasaki 1899: 435 (11)

Dryopeia hirsuta Baker 1921: 159 (1)

Tetraneura nigriabdominalis: Tanaka 1961: 73, 9 (14)

Tetraneura nigriabelominalis: Eastop 1966: 041 (4)

Tanaka (14) lists Dryopeia hirsuta Baker (1), which has been known as Tetraneura hirsula (Baker), as a synonym of nigriabdominalis.

Apterous Vivipara: Antennae, legs, siphunculi, abdominal segments VII, VIII, and cauda dusky, rest of body pale. Antennae 5 segmented, a.s. IV and V apparently coalesced. Setae on a.s. I, 3; II, 2; setae on a.s. II, 0.05 . Rostrum attaining 2nd coxae and bearing 4 accessory setae. Abdomen with a conspicuous row of lateral setae 0.16. Tarsomeres I and II fused. Wax plates inconspicuous, bearing 1 to 6 cells. Abdominal segment VIII with 2 conspicuous setae 0.15 . Cauda with 3 setae, the middle seta conspicuously shorter than the other two. Measurements of one specimen. Body 1.94. Width of head 0.46. A.s. III, 0.09 ; IV, $0.14 ; \mathrm{V}, 0.04+0.02$. R IV +V, 0.10. Hind tibia 0.29. Hind tarsus 0.0S. Siphunculi short, truncate, 0.10. Cauda 0.06 .

Alate Vivipara: Color of living specimens dark with a greenish tinge on abdomen. Cleared specimens dark on head, antennac, thorax, legs, siphunculi, cauda, and anal plate. Head with 2 small oval wax plates which are widely separated. Tarsomere I and II not fused. Tarsomere II strongly denticulate. Media of fore wings simple, not branched. Siphunculi short, truncate. Cauda bearing 3 setae, the center one being noticeably shorter than the other two. Measurements of a single specimen. Body 2.0. Width of head 0.37. A.s. III, 0.21 ; IV, 0.07; V, 0.21; VI, $0.04+0.02$. IR IV + V, 0.10 . Hind tibia 0.76. Hind tarsus 0.15. T. ch. 2-2-2. Siphunculi 0.02. Cauda 0.08.

Collections: On Digitaria decumbens Stent, (roots) Isabela, Apr. 1970 (M. Colón, M. Restrepo, A. Lugo).

On Emilia coccinea, (alates) (69-23), Cerro La Marquesa, Aguas Buenus, 1,500 ft. alt., (radio and TV towers), Mar. $\$, 1969$, (C JS, CKS, IFMI).

In flight, (69-1), Lal Torre, Batrio Boquerón, Batranquit as, Mar. s), 1969, (CFS, MIET, RW). 
On grass (alate), (69-3), La 'Torre, Barrio Boquerón, Barranquitas, Mar. j, 1969 (CFS, MISP, RW).

Unknown (69-22) (alate) El Yunque, East leak Rd. (at gate), Mar. 7, 1969 (CFS, CKS, LFM, RW).

Resting, (69-28), Florida, Mar. 10, 1969, (CFS, CKS, MEP).

New record for Puerto Rico.

\section{Toxoptera aurantiae (Boyer de Fonscolombe)}

Fig. 53

On Anthocephalus cadamba, (69-8), Catalina Nursery, El Yunque Rd., Km. 4.2, Mar. 6, 1969, (CFS, CKS, LFMI, RW).

On Calliandra surinamensis, (67-142), Agronomy Building, Agricultural Experiment Station, Río Piedras, Apr. 10, 1967, (CFS, LFM).

On Calophyllum brasiliense = Calophyllum antillanum, (67-109), MaricaoSabana Grande Rd., Km. 17.9, Apr. 4, 1967, (CFS, CKS, LFM, RW); (69-55), south shore of Lake Tortuguero, Mar. 12, 1969, (CFS, RW).

On Citus sinensis, (67-82), Mayagüez-Maricao Rd., Km. 11.6 Apr. 4, 1967, (CFS, CKS, LFM, RW).

On Clusia gundlachii, (67-107), Maricao-Sabana Grande Rd., Apr. 4, 1967, (CFS, CKS, LFM, RW).

On Coccoloba microstachya = Coccolobis oblusifolia, (69-52), Bayamón, Kim. 15.1, Mar. 12, 1969, (CFS, RW).

On Cordia alliodora = Cerdana alliodora, (69-49), end of Guilarte Forest Rd., 3,450 ft. alt., Mar. 12, 1969, (CES, Clis, LFM, SMG).

On Dendropanax arboreum, (67-54), Arecibo-Iares Rd., Iím. 34, Apr. 3, 1967, (CFS, CKS, MEP, RW).

On Didymopanax gleasonii, (67-121), Cerro Punta area, Jayuya, Toro Negro lorest, 4,000 ft. alt., Apr. 6, 1967, (ClS, CKS, LFM, RW).

On Didymopanax morototomi, (67-83), Mayagüez-MIaricano Rd., Km. 11.6, Apr. 4, 1967, (ClS, CKS, LFM, RW).

On Epidendrum secundum = Amphiglotlis secunda, (67-108), MaricaoSabama Grande Rd., Apr. 4, 1967, (CFS, CKS, LFMI, RW).

On Eugenia malaccensis = Jambosa malaccensis, $(67-95)$, road to fish hatchery, Maricao, Apr. 4, 1967, (CFS, CKS, LF MI, RW); (67-77 \& 67-78), Maỵagüez-Maricao Rd., Kim. 11.6, Apr. 4, 1967, (ClS, CKS, LFM, RW).

On Gesneria albiflora = Pentarhaphia albiflora, (67-47), Arecibo-Lares Rd., Kim. 34, Apr. 3, 1967, (CFS, Clis, MIEP, RW).

On Gomzalagunia aspicata $=$ Duggena hirsuta, $(67-20)$, El Yunque, Apr. 1, 1967, (CFS, CKS, MEP); (67-34), El Yunque, Km. 11.3, Apr. 1, 1967, (C'FS, C'LS, MLEP).

On Guarea trichilioides = Guarea guara, (67-70), Rd. No. 135), ('astañer, Apr. 3, 1967, (Cl'S, CKS, MLFP, RW). 
On Hamelia erecta, (67-ijs), Arecibo-Lares Rd., lim. 34, Apr. 3, 1967, (ClS, CliS, MLP, RW).

On Hibiscus rosa-sinensis, ((i7-25), LI Yunque Rd., Kim. 7.7, Apr. 1, 1967, (ClS, CKS, MEP).

On Ilex macfadlyenii, (69-18), East Peak Rd., El Yunque area, 2,200 ft. alt., Mar. 7, 1969, (ClS, CliS, LF MI, RW).

Iresine diffusa $=$ Iresine celosia, (67-50), Arecibo-Lares Rd., Kim. 34, Apr. 3, 1967, (CFS, CliS, MIEP, RW).

On Malpighia coccigera, (67-187), Villalba-Orocovis Rd., Irm. 1.4, Apr. 11, 1967, (ClS, CKS, LFM).

On Mammea americana, (67-93), road to fish hatchery, Maricao, Apr. 4, 1967, (CFS, CKS, LFMI, RW); (67-44), Barrio Sonadora, Aguas Buenas, on Rd. No. 792, 1,300 ft. alt., Apr. 2, 1967, (ClS, CKS, LFM).

On Myrcia fallax = Myrcia berberis, (67-177), Cerro Punta, Jayuya, Toro Negro Forest, 4,000 ft. alt., Apr. 6, 1967, (SMG, ClS, LF.M, RW).

On Myrcia splendens, (67-39), El Peñón del Collao, Cayey-Aibonito Rd., 2,000 ft. alt., Apr. 2, 1967, (ClS, CKS, LFMI).

On Nothopanax guilfoylei = Polyscias guilfoylei, (67-222), El Yunque, Apr. 14, 1967, (ClS, LF II).

On Piper aduncum, (67-128), Barranquitas-Naranjito Rd., Kim. 9.9, Apr. 7, 1967, (Cl'S, CliS, MEP); (67-19), El Yunque Rd., Kím. 2.4, Apr. 1, 1967, (CFS, CKS, MIEP).

On Piper marginatum, (67-49), Arecibo-Lares Rd., Ḱm. 34, Apr. 3, 1967, (ClS, CKS, MEP, RW).

On Rapanea ferruginea, (67-14\$), La Santa, Guavate Forest, Cayey, Apr. 10, 1967, (ClS, Clis, SMG); (67-118), Cerro Punta area JayuyaToro Negro Forest, $4,000 \mathrm{ft}$. alt., Apr. 6, 1967, (CFS, SMG, LFM, RW).

On Rosa sp., (67-150), Guayama-Cayey Rd., Kim. 17.3, Apr. 10, 1967, (CIS, CKS, SMG).

On Rubus florulentus, (67-111), Maricao, Apr. 4, 1967, (CFS, CKS, IFII, RW); (67-119), Cerro Punta area, Jayuya, 4,000 ft. alt., Apr. 6, 1967, (CFS, S.IG, LI MI, RW).

On Symplocos micrantha, (67-120), Cerro Punta area, Jaỵuya, 4,000 ft. alt ., Apr. 6, 1967, (SMG, ClS, IJAI, RW).

On Tabebuia haemantha, (67-110), Maricao, Apr. 4, 1967, (ClS, CKS, $\mathrm{LI} \times \mathrm{N}, \mathrm{RW}$ ).

On Theobroma cacao, (67-76), Milyigüez-Maricao Rd., Kim. 11.6, Apr. 4, 1967, (CFS, CKS, LF.2I, RW).

\section{SLMMARY}

This is a supplement to the "Aphididae of Puerto Rico." (12) The key to the "Aphididae of Puerto Rico" has been revised. A new lield kiey to the Apterous Aphididae of Puerto Rico is also included, with a photograph 
of each species. Five additional species are recorded: Capitophorus hippophaes jaranicus H. R. L., Macrosiphum rosae L., Picturaphis puertoricensis Smith, Rhopalosiphum padi L, and Tetraneura nigriabdominalis (Sasaki), bringing the tot al known species of aphids from Puerto Rico to 51. Macrosiphum mesosphaeri Tissot is listed as a new synonym of Macrosiphum (Sitobion) salviae Bartholomew.

\section{RESUMEN}

Este trabajo es un suplemento a la publicación intitulada "Aphididae of Puerto Rico" (12). La clave para los áfidos de Puerto Rico se ha revisado. También se incluye en este trabajo una clave de campo para los áfidos de formas aladas de Puerto Rico y se ilustra cada especie con una fotografía. Se informan por primera vez cinco especies adicionales: Capitophorus hippophaes javanicus H. R. L., Macrosiphum rosae L., Picturaphis puertoricensis Smith, Rhopalosiphum padi L. y Tetraneura nigriabdominalis (Sasaki), elevando a $\overline{5} 1 \mathrm{el}$ total de especies de áfidos conocidos de Puerto Rico. Macrosiphum mesosphaeri Tissot se incluye como un sinónimo de Macrosiphum (Sitobion) salviae Bartholomew.

\section{LITERATURE CITED}

1. Baker, A. C., An undescribed aphid injurious to rice in the Philippine Islands, Calif. Dep. Agr. Mon. Bull. 10, 159-60; figs. 33-55, 1921.

2. Bartholomew, P. S., Six new species of aphids, with records of other species new to California, Ann. Entomol. Sor. Amer. 25: 713-29, Pls. I-II, 1932.

3. Böruer, C., Europae centralis Aphides. Die Blattläuse Mitteleuropas. Namen, Synonyme, Wirtspflanzen, Cienerationszyklen, Mitt. Thur. Bot. Ges. 3: 1-488, 1952.

4. Eastup, V. F., A taxonomic study of Australian Aphidoidea (Homoptera), Aus(ral. J. Zool. 14: 399-592, figs. 1-192, 1966.

5. Ilille R is Lambers, J), Cont ributions to a monograph of the Aphididae of Europe, V. Temminckia 9: 1-176, Pls. I-VI, text figs. 1-10, 1953.

6. Iottes, F. C. and T. H. Frison, The plant lice, or Aphididae, of Illinois, Ill. Dep. Regist. Educ. Div. Natur. Hist. Surv. Bull. 19, 121-4t7, Pls. I- I, text figs. 1-50, 1931.

7. Limnaeus, C., II. Hemiptera. Systema Naturae per regua tria naturae, secundum classes, ordines, genera, species, cum characteribus, differentiis, syunuymis, locis., Editio decima, reformat a $1: 1-824(451-453), 1758$.

8. Liogier, A. II., (Brother) Nomenclatural changes and additions to Britton and Wilson's "Florn of Porto Rico and the Virgin Islands", Rhodora 67 (772) (reference from Martorell in litl.), 1965.

9. - -.., Further changes and additions to the Flora of Porto Rico and the Virgin Islands, Rhodora 69 (779), 1967.

10. Mason, P. W., A new root aphid (Homoptera: Aphiidae), Proc. Entomol. Soc. Hiash. 39 : 166-(i7, fig. 1, 1937.

11. Sasaki, C., Toroptera miabdominalis Sasaki., Mantal of Japanese Insect Pests of Crops 1899: (202) (reference from Eastop 196(i), 1899. 
12. Smith, C. F., L. F. Martorell, and M. E. Pérez-Escolar, Aphididac of Puerto Rico, Univ. P. R. Agr. Exp. Sta. Tech. Pap. 37, 1-121, figs. 1-48, 1963.

13. Smith, C. F., Notes on the genus l'icturaphis Blanchard with a new species from Puerto Rico (Aphididae: Homoptera), J. Ayr. Univ. P. R. 54 (4): 683-88, 1970.

14. Tanaka, T., The rice root aphids, their ecology and control. Spec. Bull. Coll. Agr. Utsunomịa Univ. 10, 1-83, Pls. I-XIII, text figs. 1-35, 1961.

15. Theobald, F. V., African Aphididae. Part II. Bull. Entomol. Res. (i, 103-53, figs. $1-38,1916$.

16. Thomas, C., Noxious and beneficial insects of the state of Illinois, Rep. State Entomol. (IIl.) 8, 1-212, i-x, figs. 1-47, 1879.

17. Tissot, A. N., Two new aphids of the Tribe Macrosiphini, Fla. Entomol. 18: 17-23, Pl. I, 1934.

18. Wolcott, G. N., "Insectac Borinquenses." A revised amnotated check-list of the insects of Puerto Rico, J. Alyr. Univ. P. R. 20: 1-(600, illus. (111-19), 1936.

19. - - Supplement to "Insect ae Borinquenses", J. A rr. Univ. P. R. 25: 33-158, 1941. 

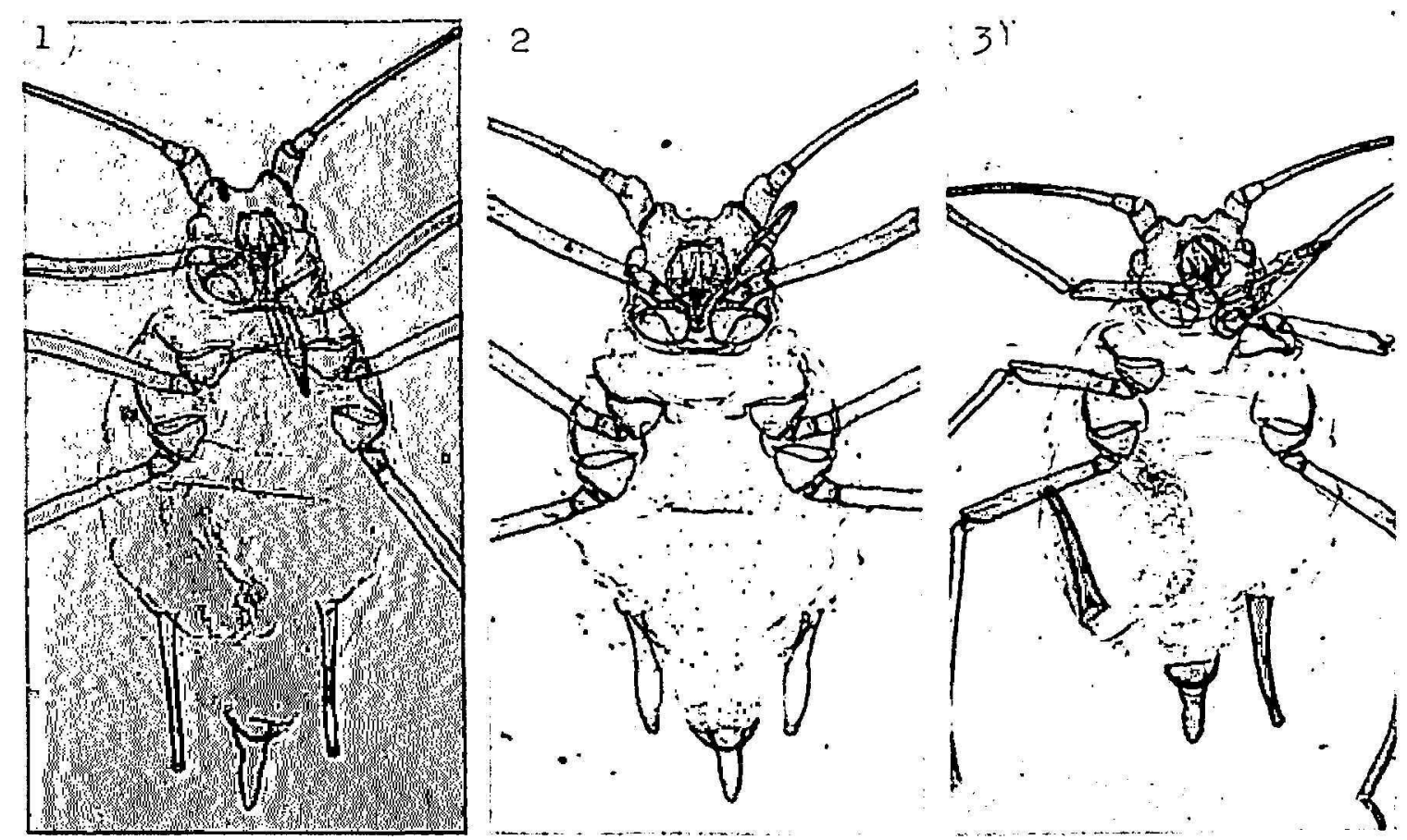

FIG. 1.-Aryrthosiphom bilenticola Simith, $22 \times$, Coll. $177-97$.

Fig. 2.--Amphorophora commelinensis simith, $30 \times$, Coll. 67-21.

FIG. 3.-A phis roreopsidis (Thomas), $30 \times$, Coll. $67-1+3$.
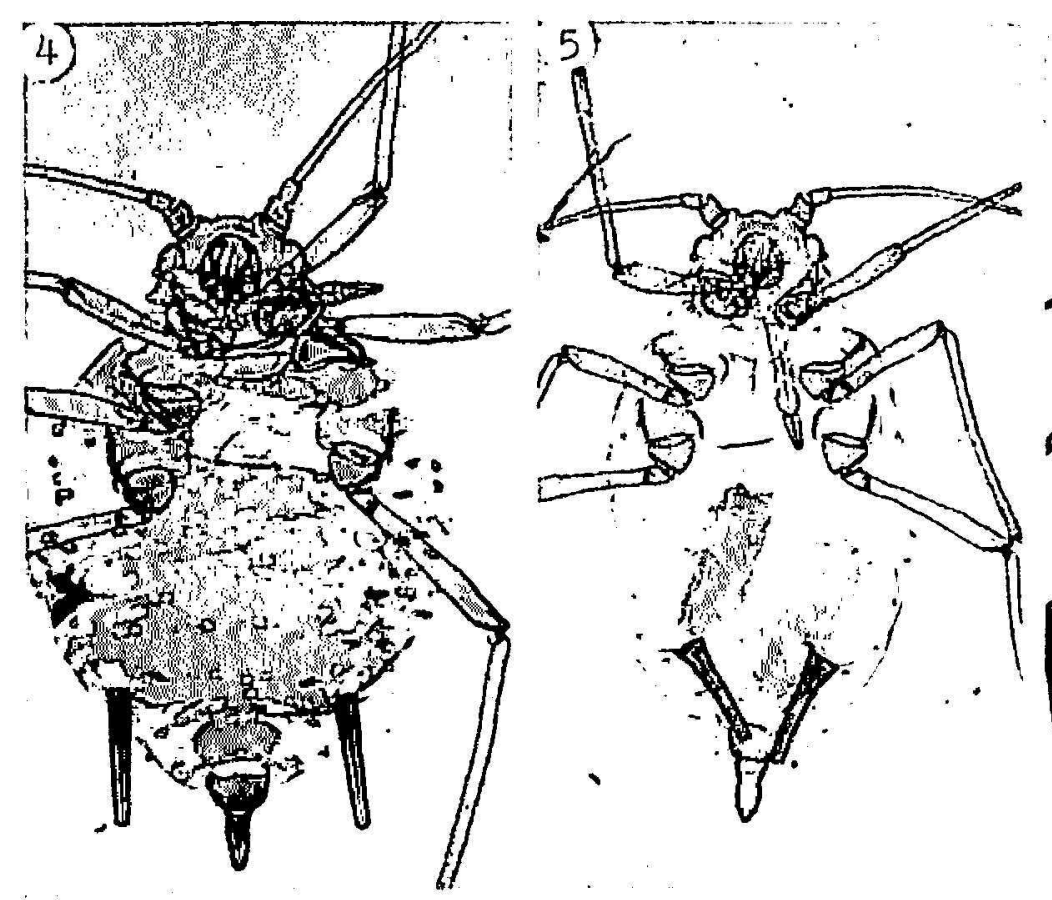

6
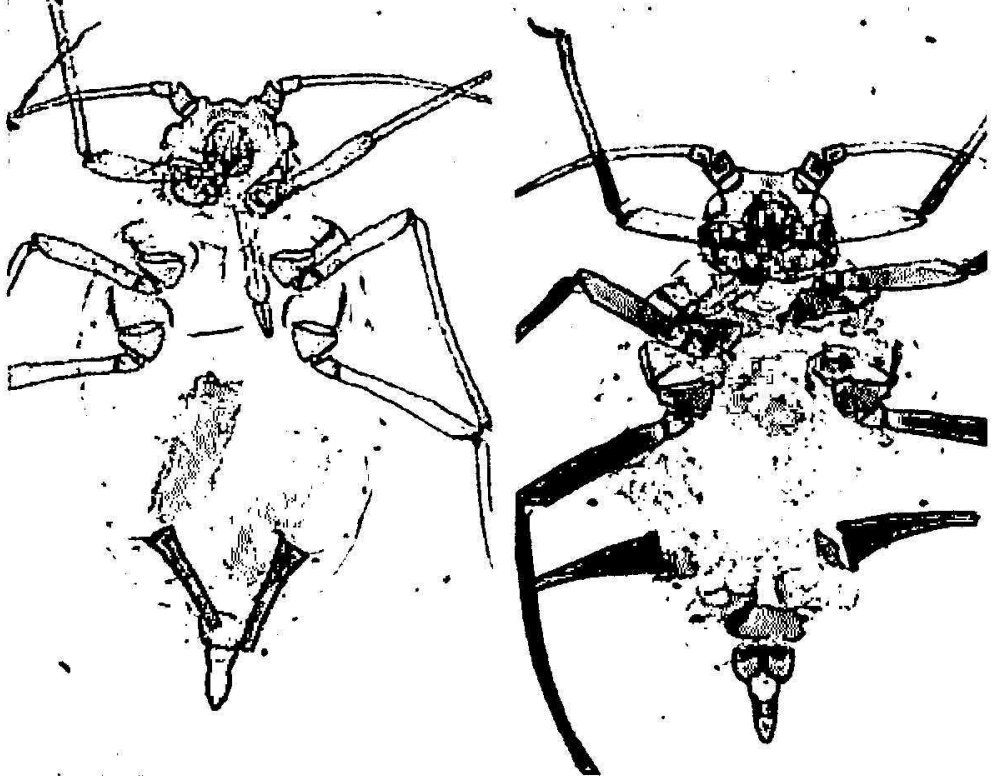

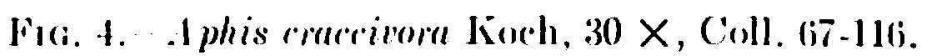

Fuc. 5. Iphis gessypui (ilover, $30 \times$, ('oll. (i)-(ii).

Fiti. (i. I phis illinoisensis shimer, $30 \times$, Coll. (i0-116. 

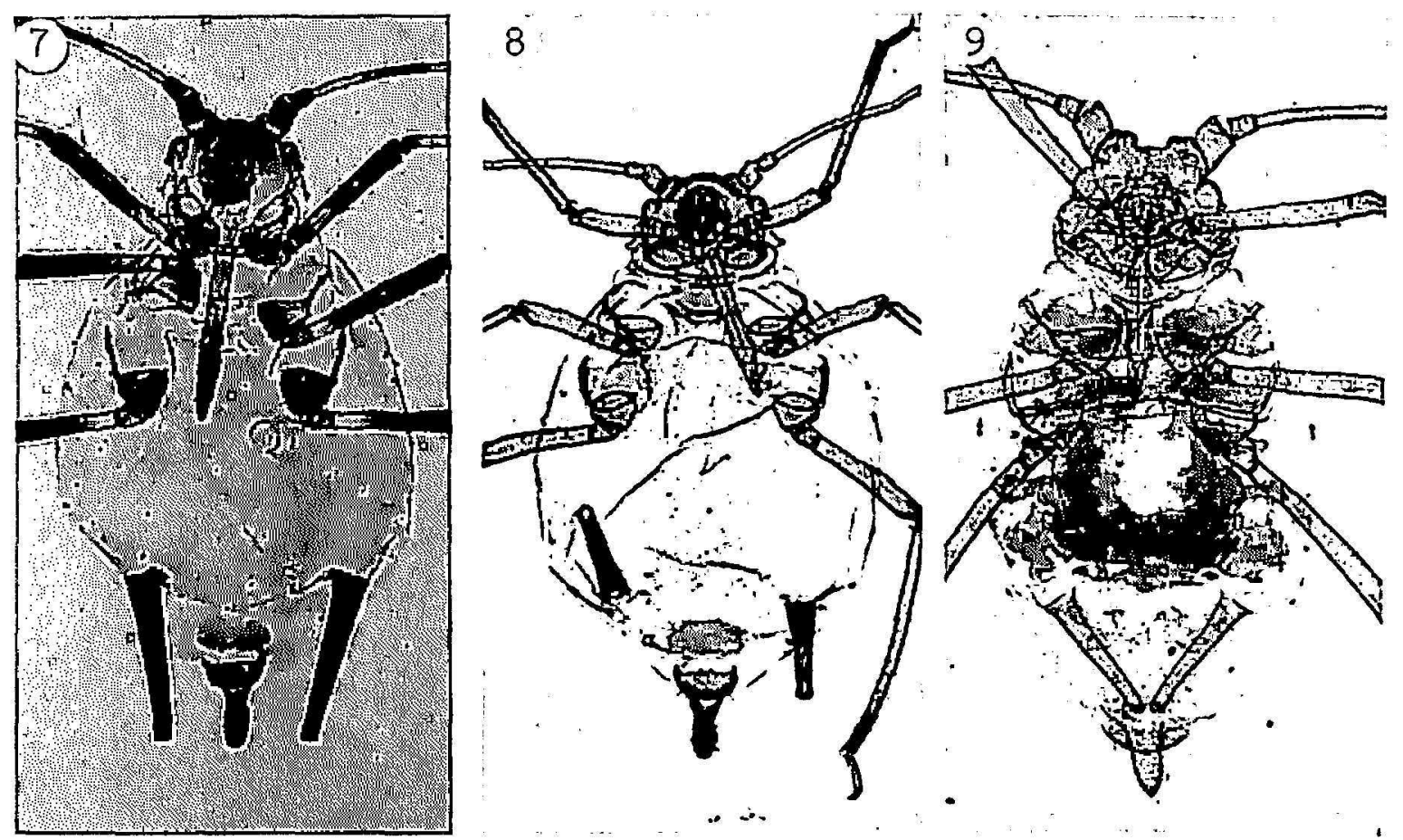

Fig. 7.-A phis nerii Boyer de Fonscolombe, $17 \times$, Coll. 67-40.

Fig. 8. - A phis spiraecola Patch, $30 \times$, Coll. $67-74$.

FIg, 9.-Aulacorthum (Neomyzus) rirrumfexum (Buckton), $30 \times$, Coll. 60-123.
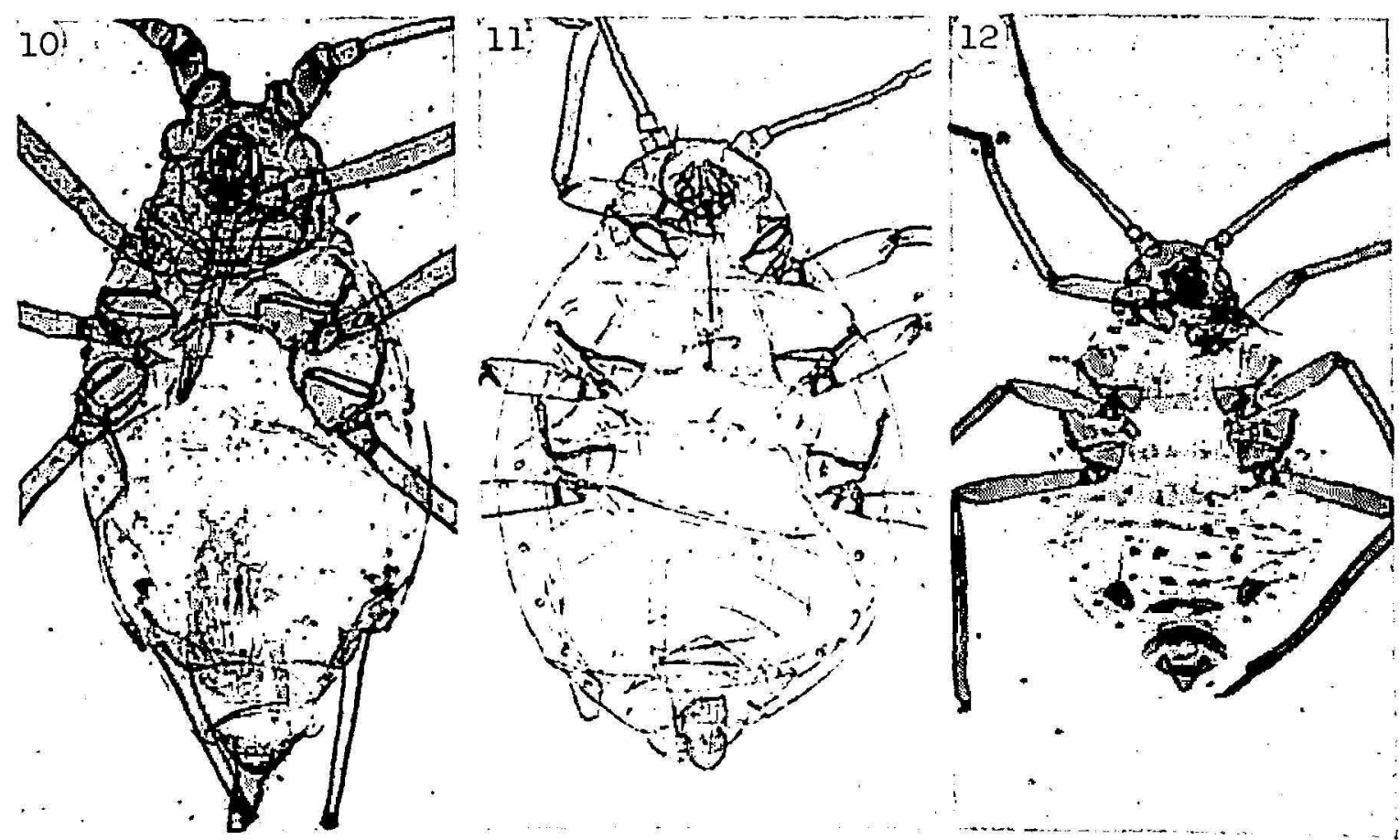

Fig. 10. Inlacorthum solami (Kalt.), $30 \times$, Coll. (i7-71.

Fic. 11. Brachycaudus helichrysi (Kalt.), $30 \times$, on Prumus domestica, Bologna, Italy, May 24, 1938, (M. Martelli).

Fia. 12. Brevicoryne brassicae (L.), $17 \times$, Coll. (i)-336. 

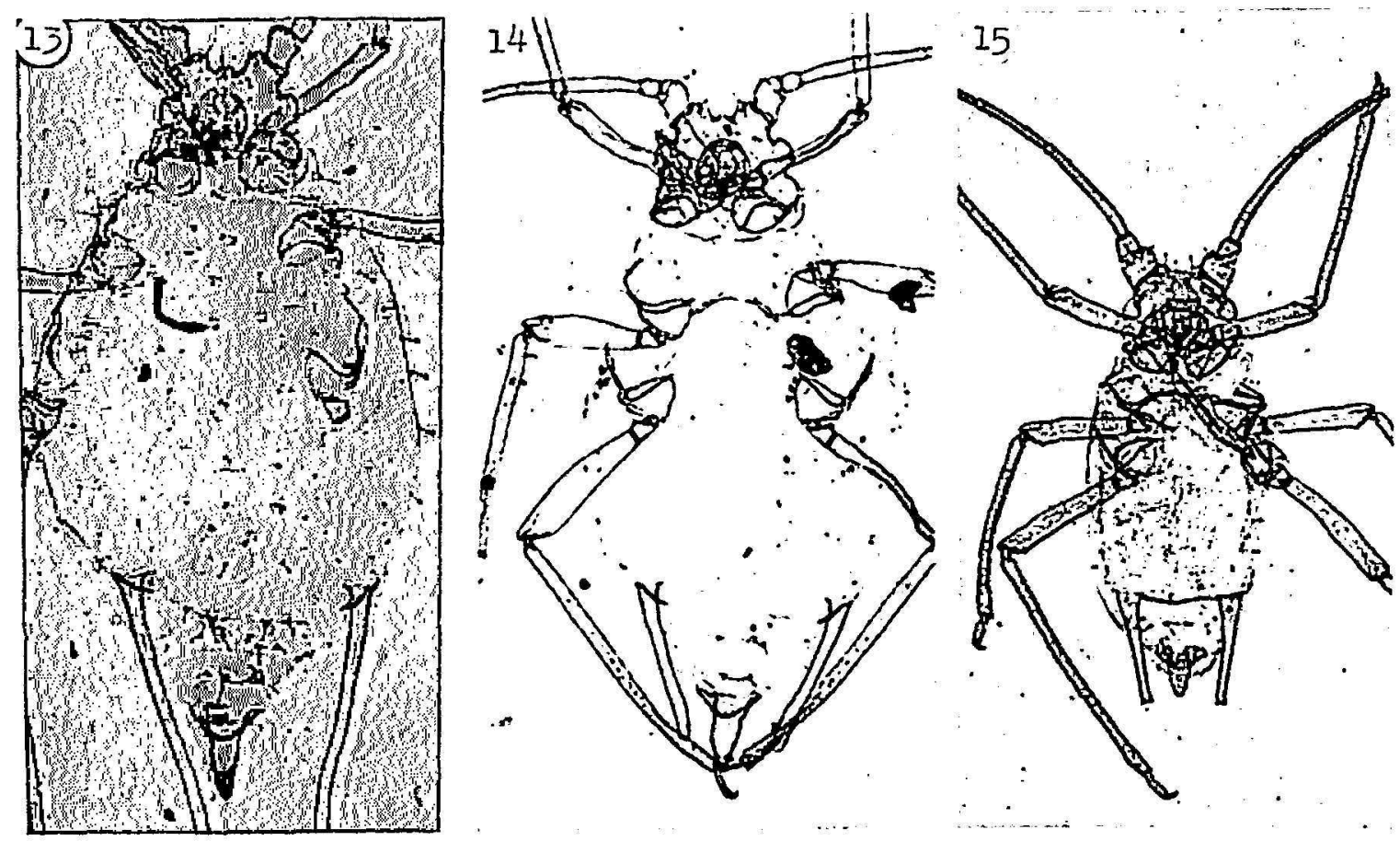

F1G. 13.-C'apitophorus elacagni (Del Cinercio), $30 \times$, Coll. 67-153.

Frg. 14.-Capilophorus hippophaes subsp. javanicus II. R. L., $30 \times$, Coll, (67-192.

FIG. 15.-C'apilophorus (Penlatrichopus) minor (Forbes), $30 \times$, Coll. 59-1549 on wild strawherry, Blowing Rock, N. C., November 11, 1959, (CFS).

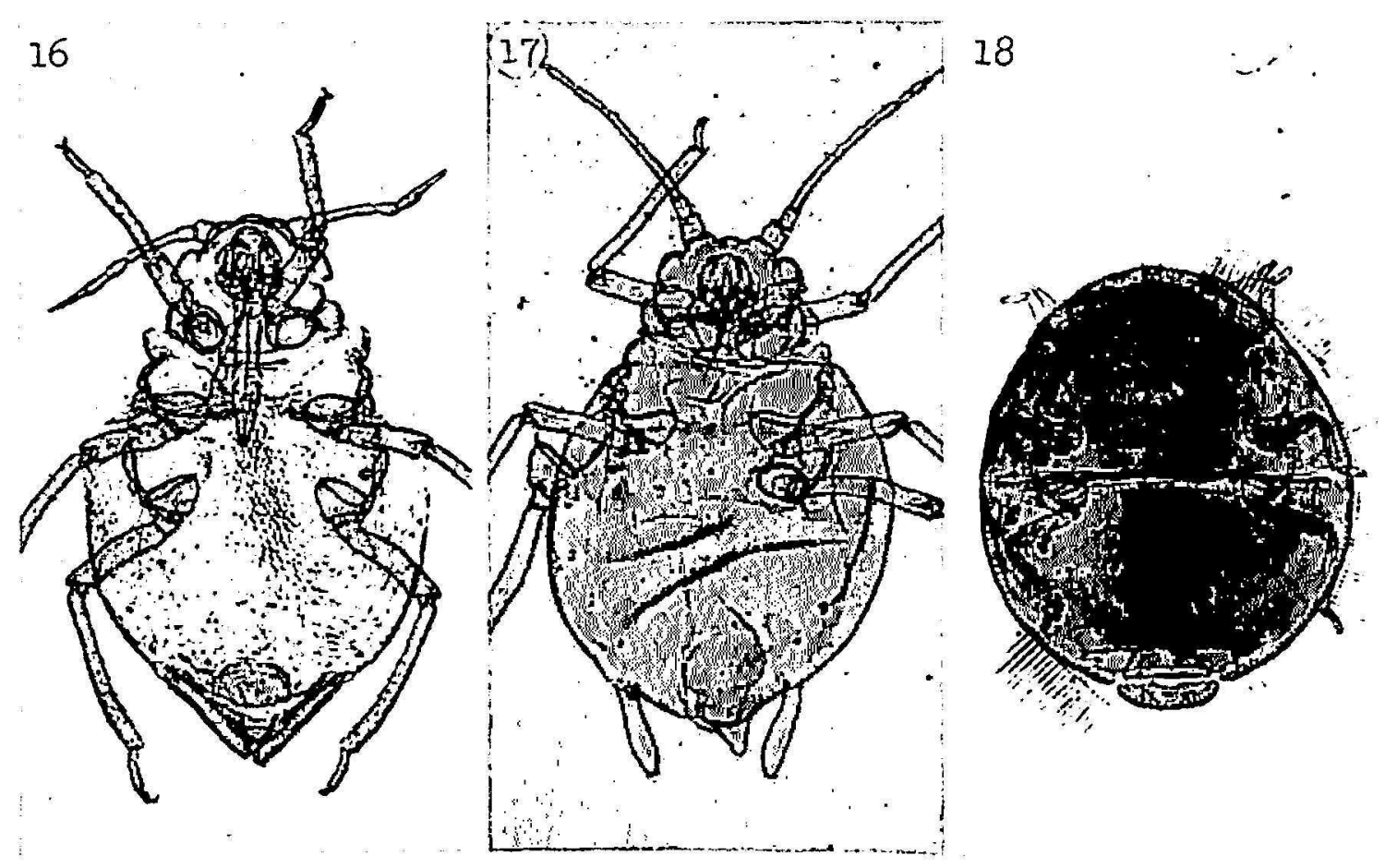

Fra. 16. Carolinaia raricis Wilson, $30 \times$, Coll. $1 \mathrm{j} 7-40 \mathrm{H}$.

Fig. 17. ('arolinaia ryperi Ainslie, $30 \times$, ('oll. 199-7.

Fic. 18. Cerataph is orchidearum (Westwood), $30 \times$, Coll. (i7-197. 


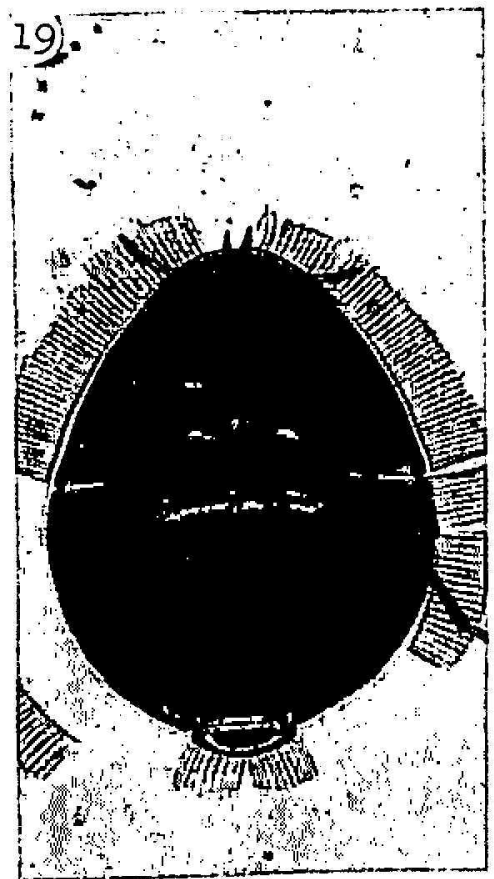

20

$21:$
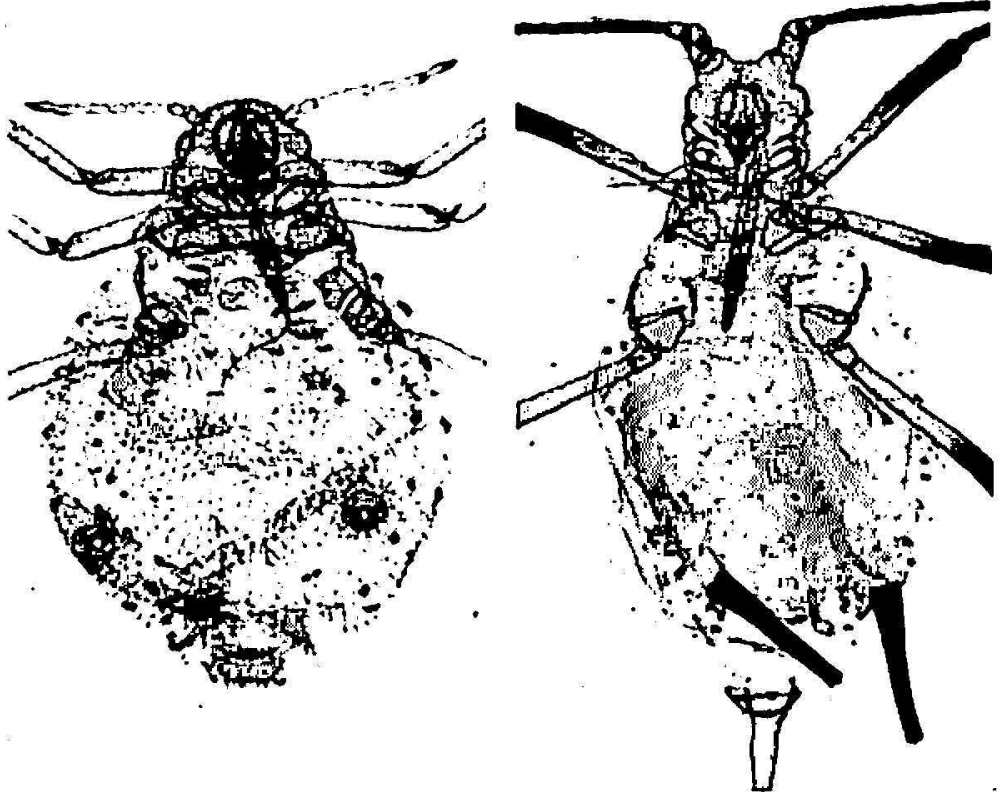

Fı, 19. - Ceralaphis variabilis II. R. I., $30 \times$, Coll. 67-141.

Fia. 20). ('inara lujafilina (Del (inercio), $17 \times$, Coll. 66i-290 on arborvitae, Provo, ltah, September 18, 1966, (CFS and li. Nelson).

Fig. 21.-Dactynotus ambrosiac (Thomas), 17 X, Coll. (67-42.
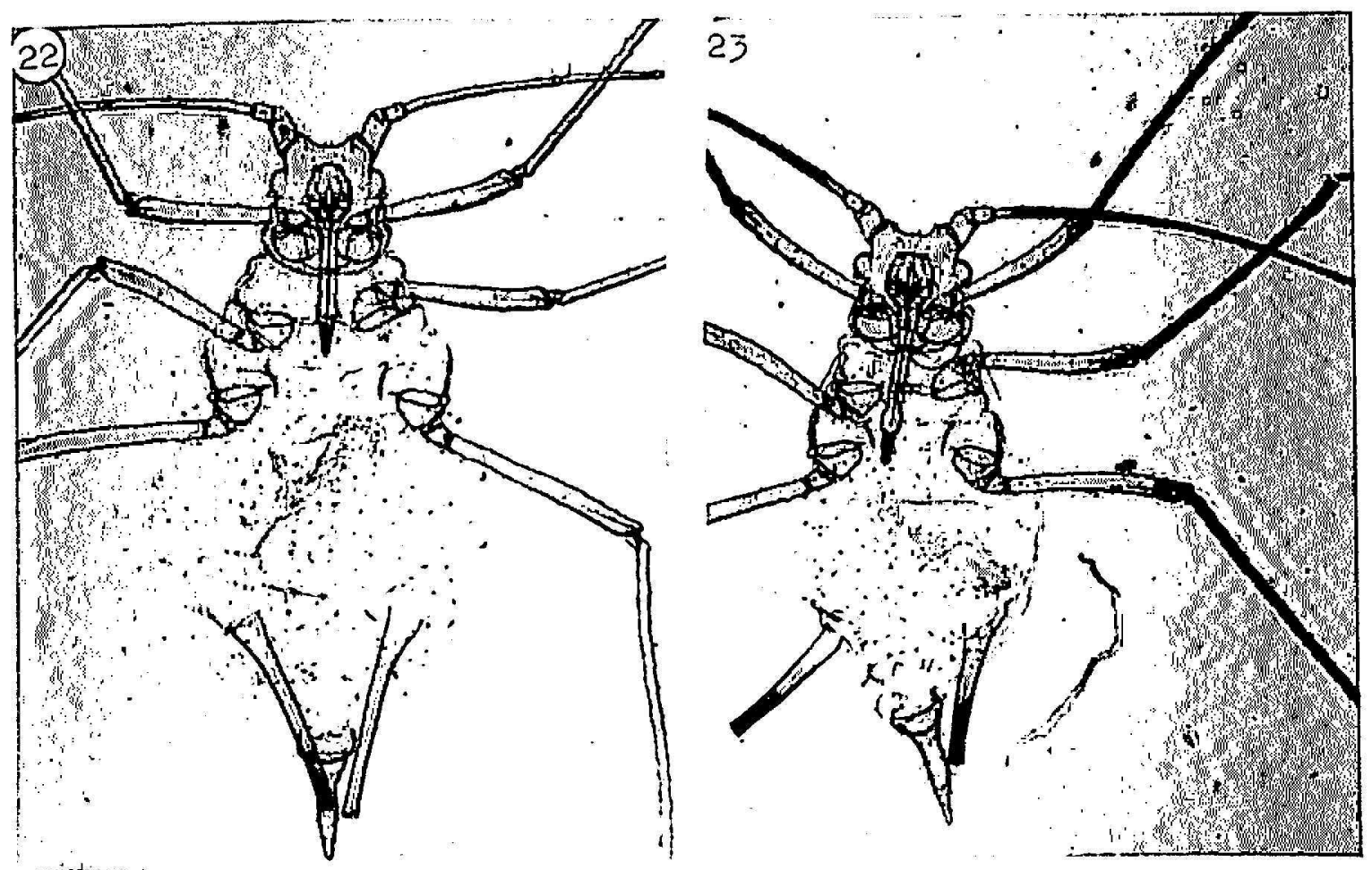

Fi(i. 22. Duclynotus erigeronensis (Thomas), $22 \times$, Coll. (67-178.

Fiti. 23. Darlynotus graricornis (Patch), $22 \times$, Coll. (69-78. 

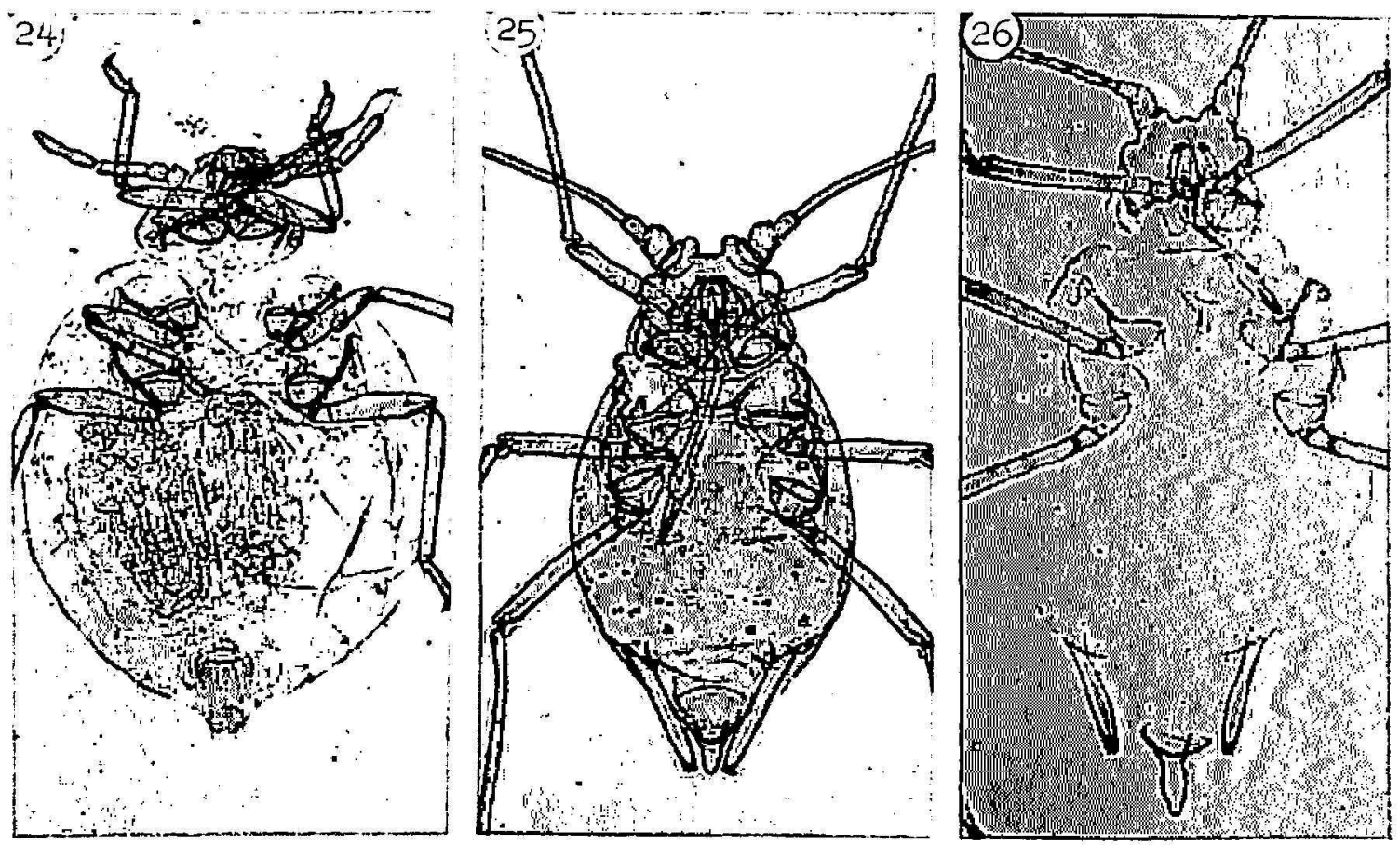

Fig. 24.-Geopemphigus floccosus (Moreira), $30 \times$, on Moonvine (roots), Belle Glade, Florida, October 17, 1959, (C. E. Seilen).

Fıg. 25.-Hyalomyzus jussiacae Smith, $30 \times$, Coll. (i7-26.

Fig. 26.--Hyperomyzus lartucae (L.), $22 \times$, Coll. li7-112.
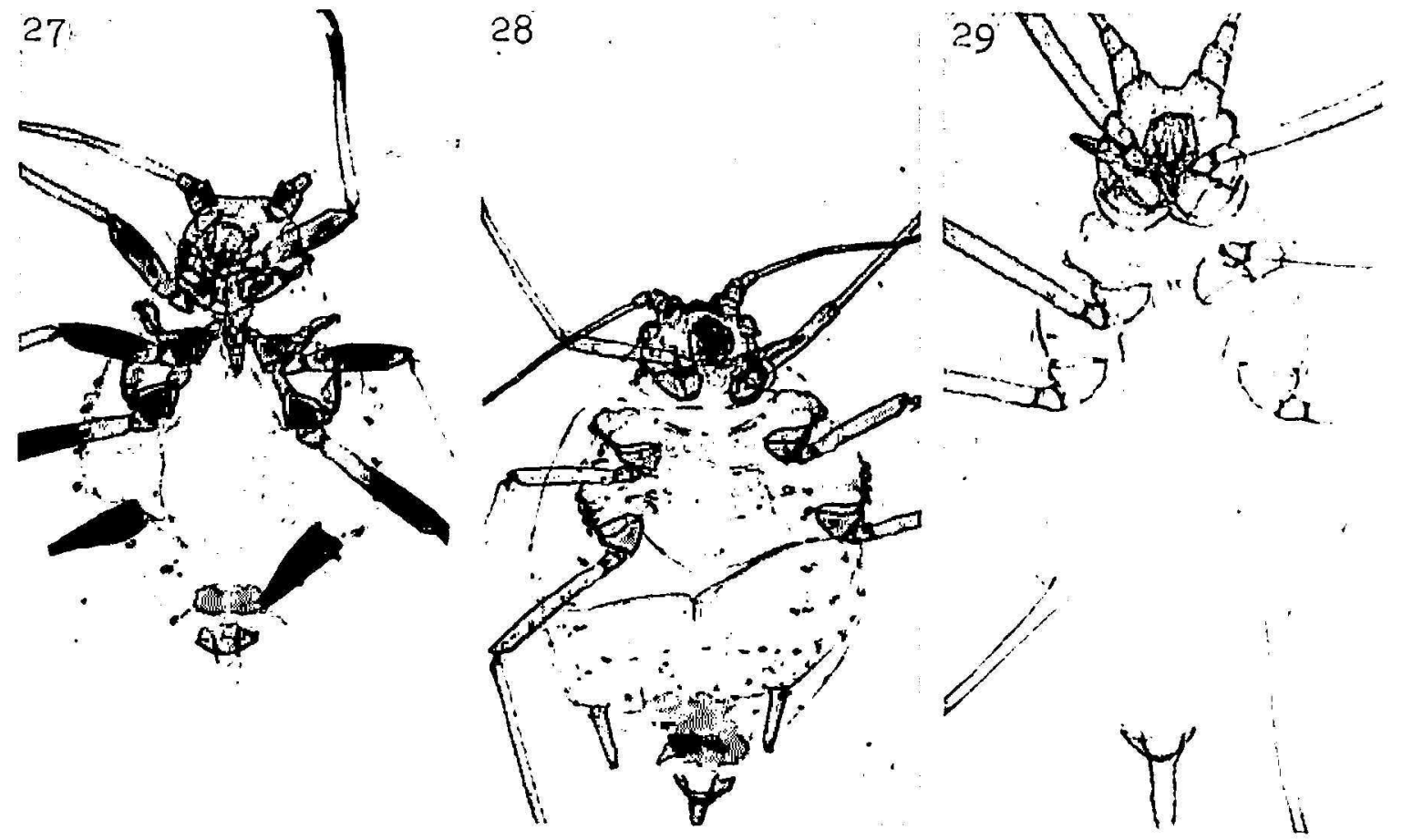

Fic: 27. Hysteroncura seturine (Thomas), $22 \times$, Coll. 5!)-25 on wild plum, West lind, N. (., April 2t, 195!), (CF's).

Fir. 28. Lipaphis pseudobrassirae (I)avis), 22 ×, on "dock", Knox Co., Tronnessec, Derember + , 196i(i, (C. (). Pless).

Fr(: 29. Macrosiphum euphobbiue (Thomas), $17 \times$, Coll. ci7-(iz. 

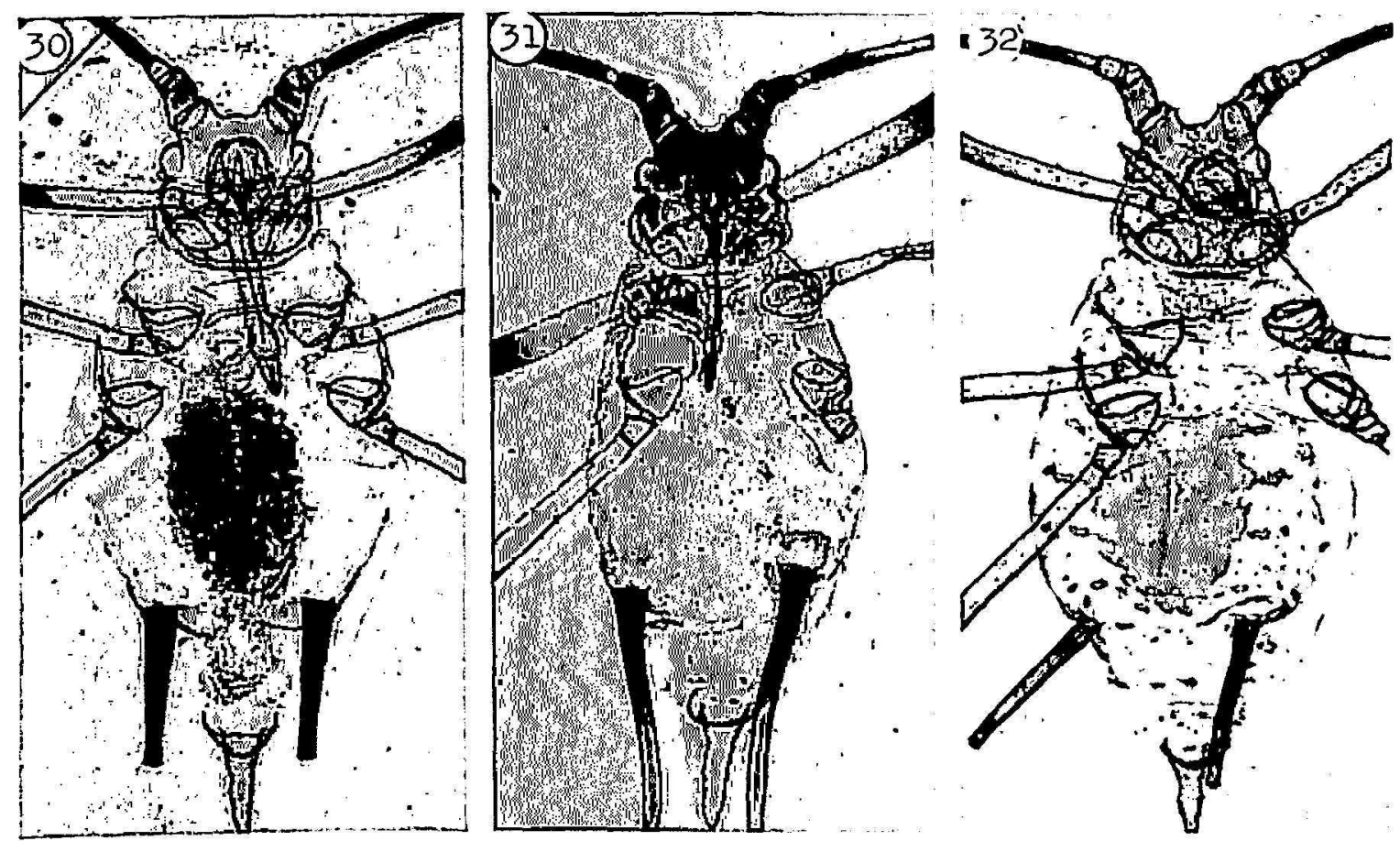

FIG. 30.-Macrosiphum (Silobion) huteum (Buckton), $22 \times$, Coll. 67-213.

FIG. 31.-Macrosiphum rosae (I.), $22 \times$, Coll. 69-44.

Fig. 32.-Marrosiphum (Silobion) salviae Bartholomew, $22 \times$, Coll. 69-158.
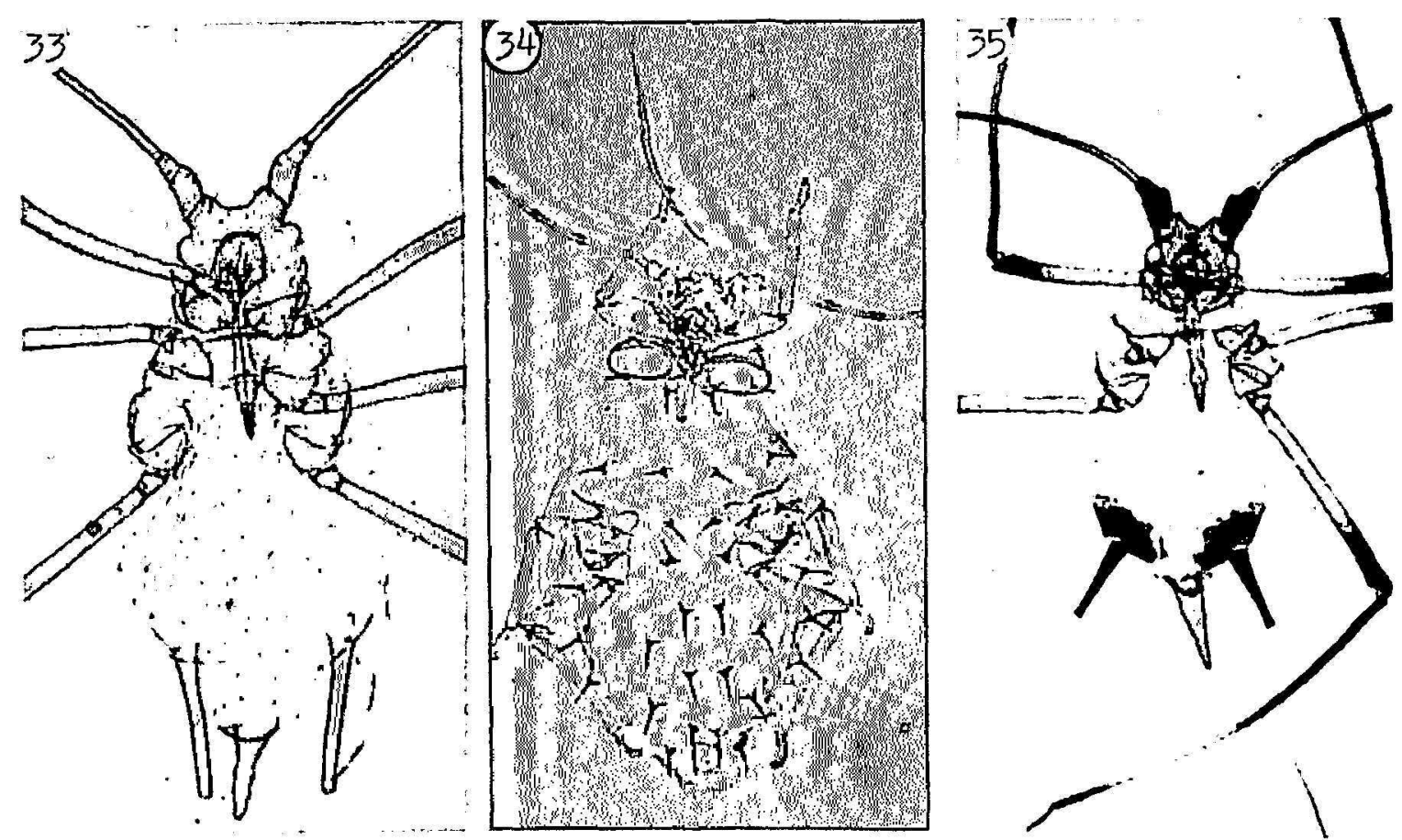

Fis. 33.--Marrosiphum (Sitobion) salciae Bartholomew, $22 \times$, Coll. (i7-126.

fina. 34. Ielanorall is kahaualuokalani (Kirkaldy), 30 X, nymph on Lagorstroemia sp., Raleigh, N. C., Neptember 2ti, 1969, (CFS).

Fic. 35. Wicroparsus olicei Śmith and Tuatay, $17 \times$, Coll. 5!-335A. 

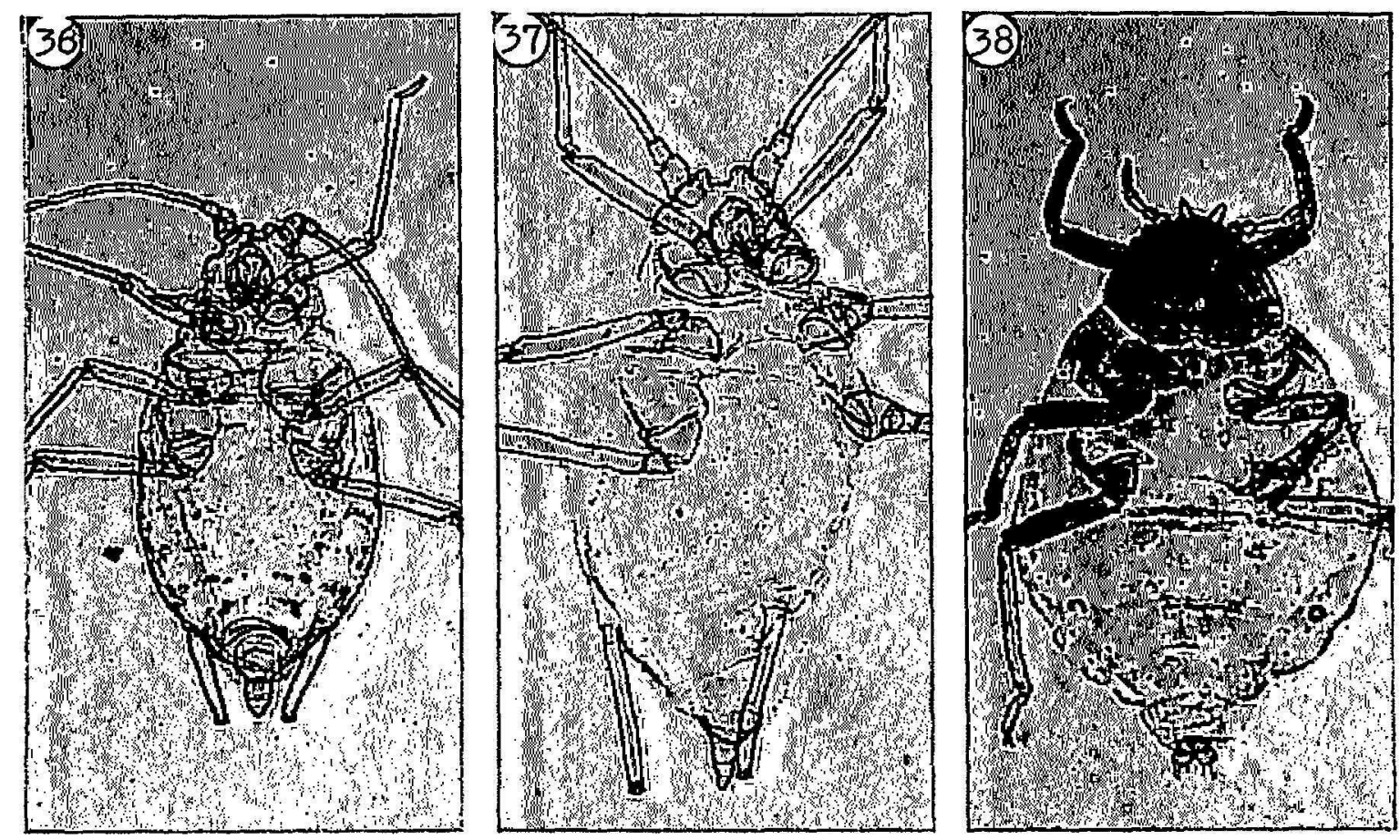

Fig. 36.-Myzus ornatus Laing, $30 \times$, Coll. 67-177.

Fia. 37.-Myzus (Nectarosiphon) persicae (Sulzer), $30 \times$, Coll. 67-1C6.

Fig. 38.-Orcyma panicola Takahashi, $30 \times$, Coll. 67-32.
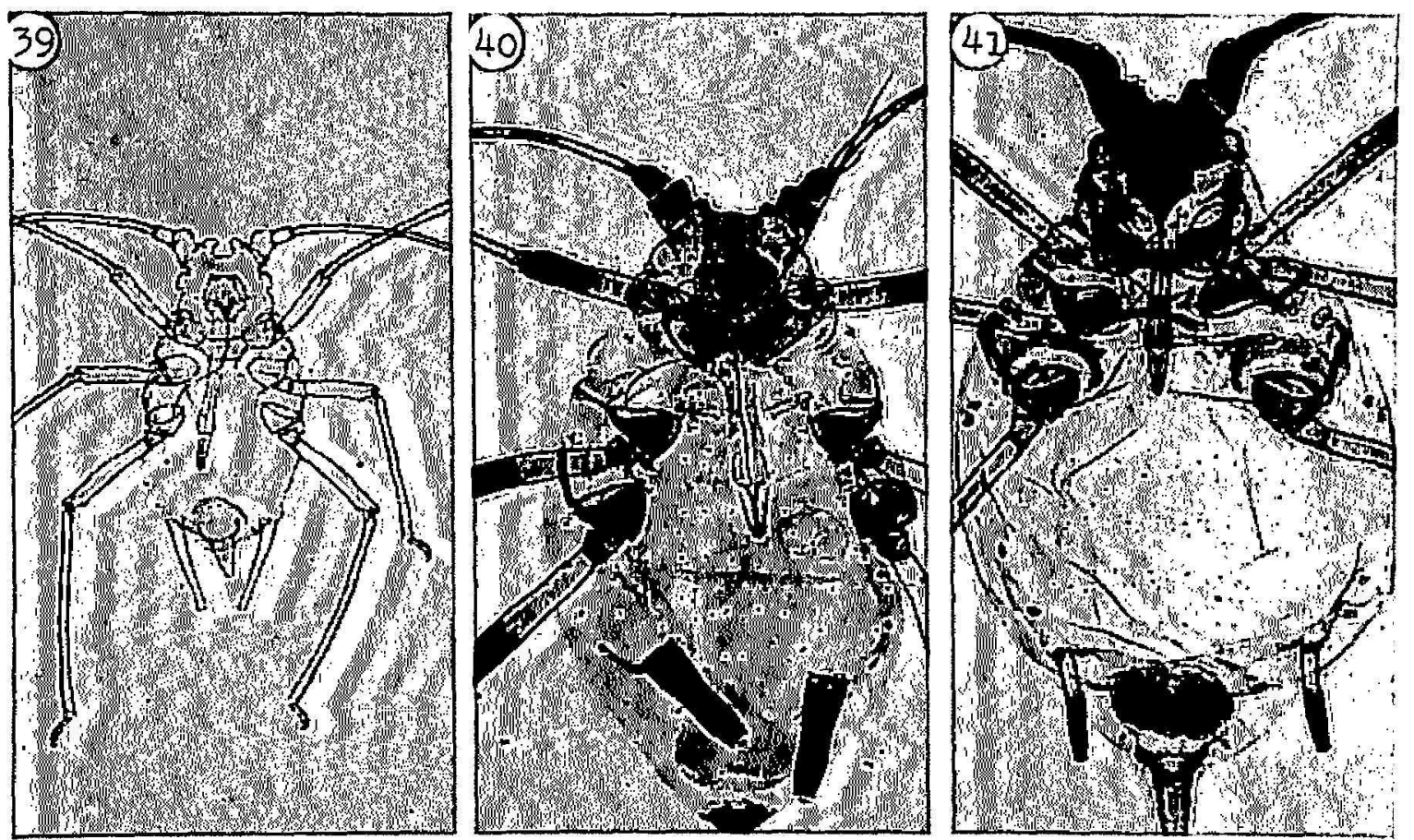

Fıg. 39.-Onatus crataegarius (Walker), $30 \times$, Coll. (67-132.

Fig. 40.-Pentalonia nigronervosa Coquerel, $30 \times$, Coll. (10-146.

F1(i. 41.--Picturaphis brasiliensis (Moreira), $30 \times$, Coll. (67-149. 

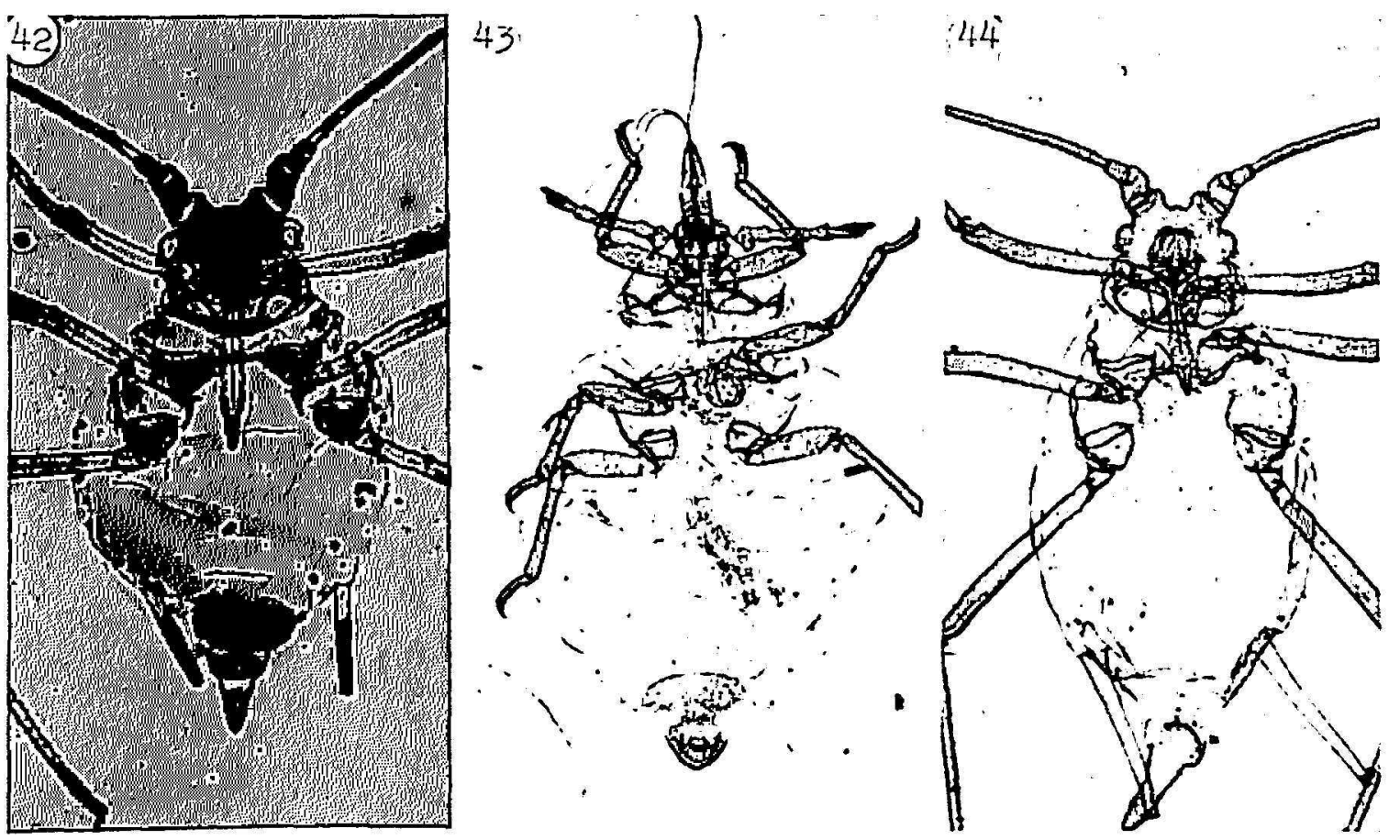

Fig. 42.--Picturaphis puertoricensis Smith, $30 \times$, Coll. 69-(65(12).

Fig. 43.-Prociphilus erigeronensis (Thomas), $22 \times$, Coll. (00-1136 on Bidens sp. (roots) Reedy Creek Park, Raleigh, N. C., October 12, 1960, (CFS).

Fug. 4t.- Rhodobium porosum (Sanderson), $22 \times$, Coll. 67-155.
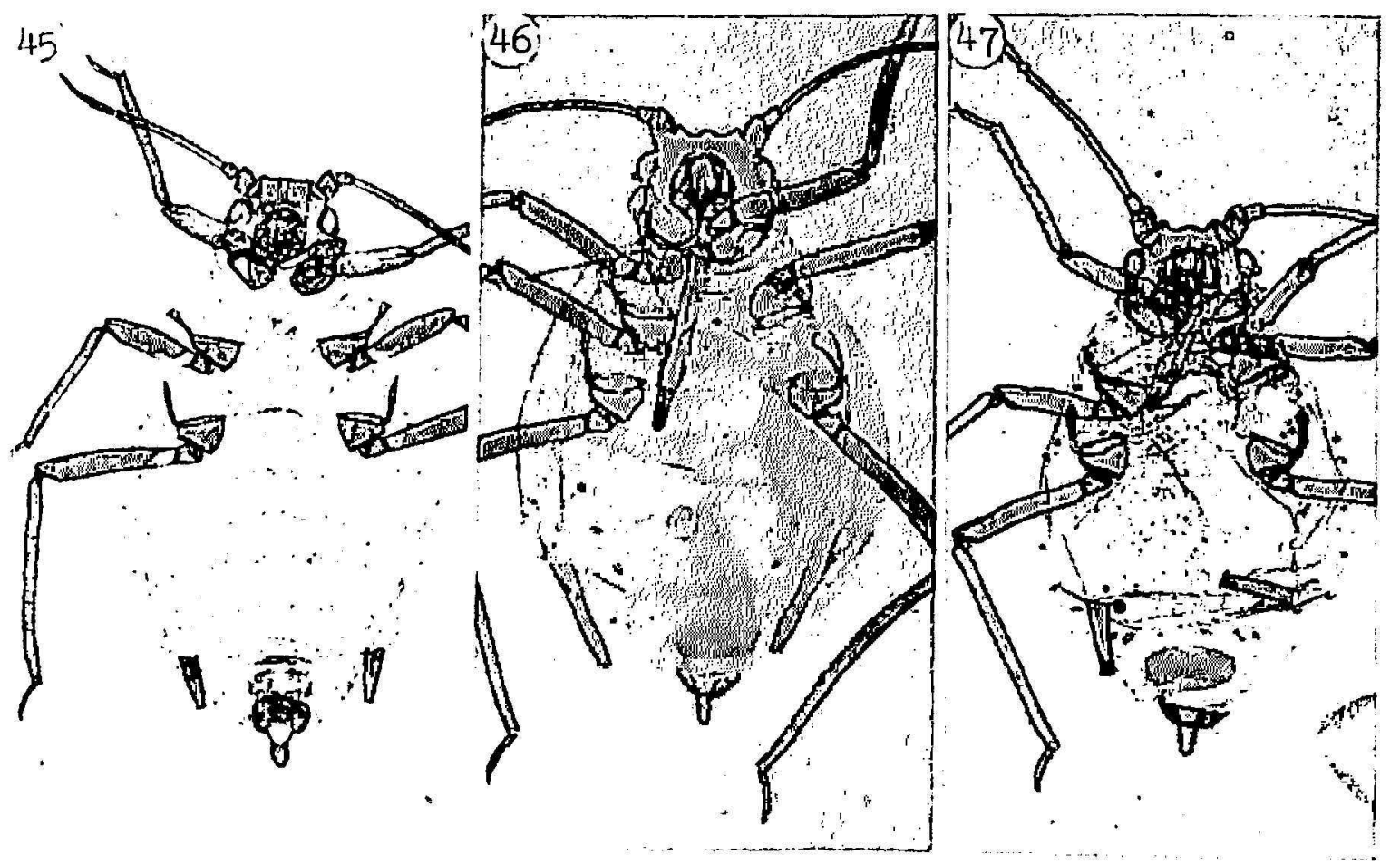

Fici. 45.-Rhopalosiphum maidis (Fitch), $22 \times$, Coll. 5+-1.

Fis. Hi. Rhopalosiphum nymphaeae (L.), $22 \times$, Coll. (i7-80.

Fisi. 47. Rhopalosiphum padi (L.), $30 \times$, Coll. 1i7-210. 

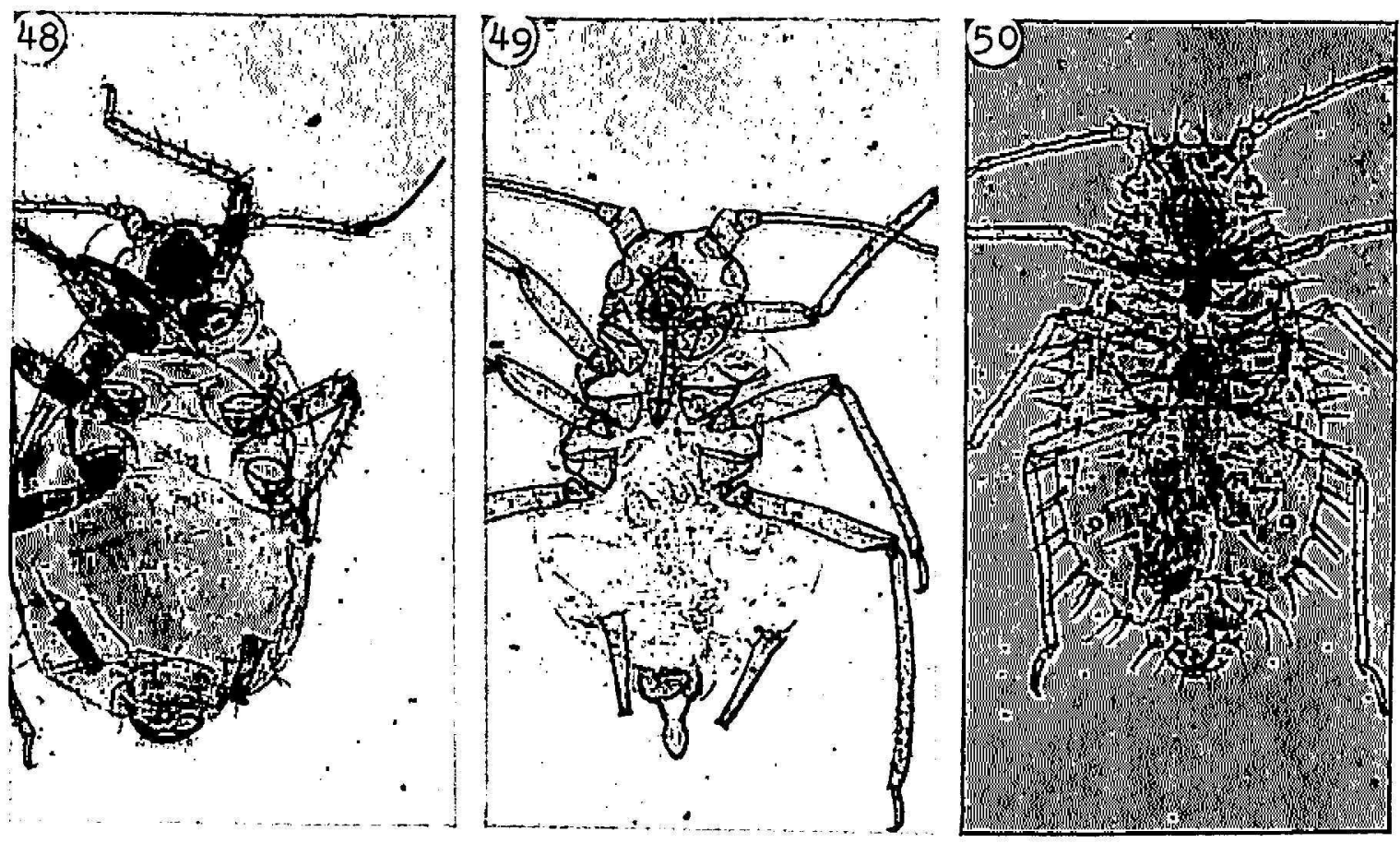

Fıg. 48.-Rhopalosiphum rufiabdominalis (Sasaki), $30 \times$, Coll. 69-66.

FIG. 49.-Schizaphis graminum (Rondani), $30 \times$, Coll. 60-174.

Fia. 50.-Sipha flava (Forbes), $30 \times$, Coll. 67-27.
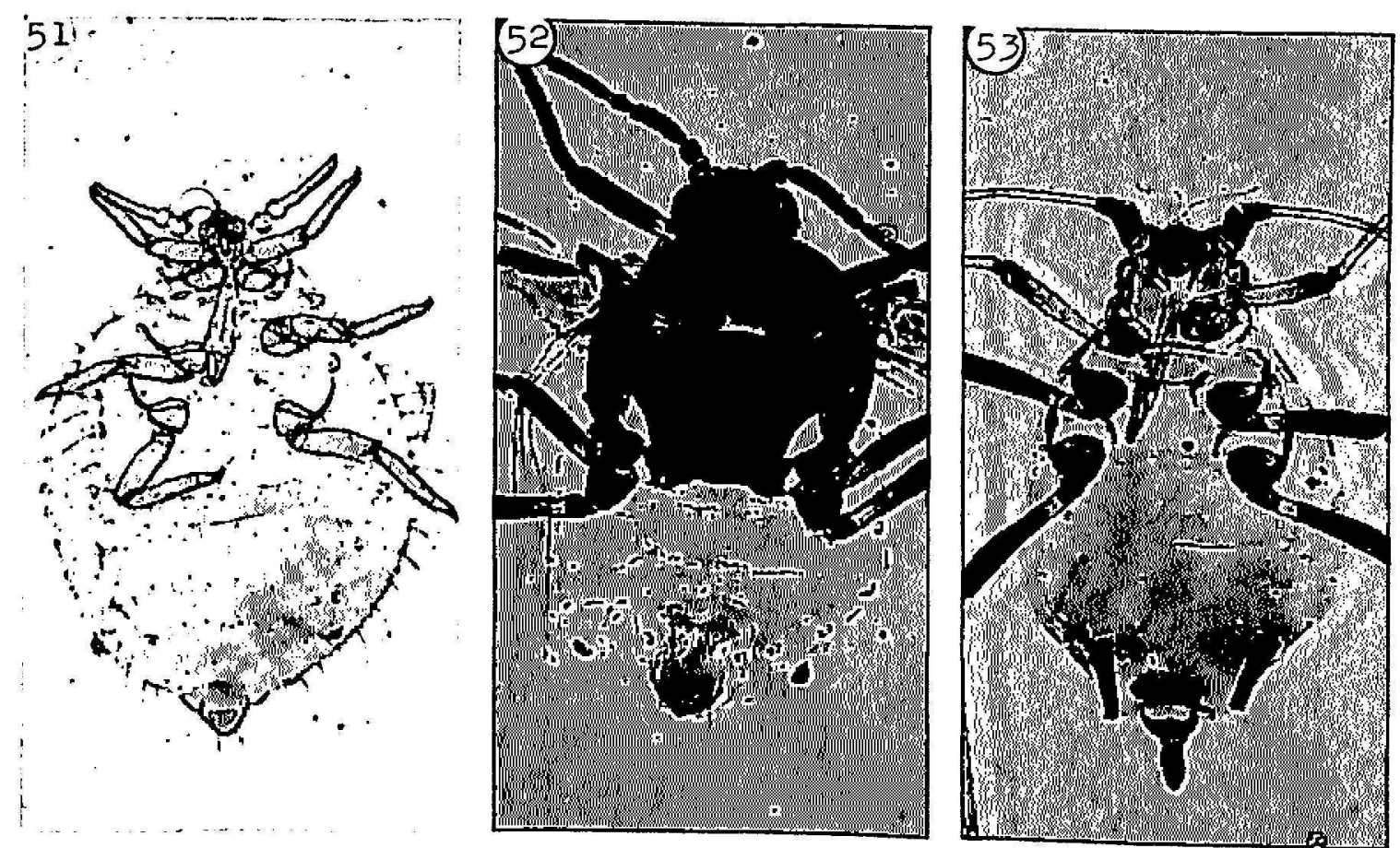

FIG. 51.--Tetruncura nigriabdominalis (Nasuki), $30 \times$, Coll. IIA 318 on Ixophorus unisetus, Sau Juan, Honduras, June 6 , 19102, (C. Lvers).

Fig. 52.-Tetraneura nigriabdominalis (S'asaki), alate $30 \times$, Coll. (69)-28.

Fia. 53.- Toroptera anrantiae, (Boyer de Fonscolombe), $30 \times$, Coll. $67-77$. 


\section{INDEX TO GENERA AND SPECIES}

Generic and subgeneric names printed in boldface type, specific names in roman; names in synonymy are in italics.

\author{
Acyrthosiphon \\ bidenticola \\ ambrosiae; Dactynotus \\ Amphorophora \\ commelinensis \\ Aphis \\ coreopsidis \\ craccivora \\ gossypii \\ illinoisensis \\ nerii \\ padi \\ spiraecola \\ Aulacorthum \\ circumflexum \\ solani \\ Aulacorthum (Neomyzus)
circumflexum \\ aurantiae; Toxoptera \\ bidenticola; Acyrtosiphon \\ Brachycaudus \\ helichrysi \\ brasiliensis; Picturaphis \\ brassicae; Brevicoryne \\ Brevicoryne \\ brassicae \\ Capitophorus \\ elaeagni \\ hippophaes javanicus \\ minor
}

Capitophorus (Pentatrichopus) minor

caricis; Carolinaia

Carolinaia

caricis

cyperi
Cerataphis

orchidearum

variabilis

Cerosipha

subterranea

Cinara

tujafilina

circumflexum; Aulacorthum (Neomyzus)

commelinensis; Amphorophora

coreopsidis; Aphis

craccivora; Aphis

crataegarius; Ovatus

cyperi; Carolinaia

Dactynotus

ambrosiae

erigeronensis

gravicornis

Dryopeia

hirsuta

elaeagni; Capitophorus

erigeronensis; Dactynotus, Prociphilus, Tychea

euphorbiae; Macrosiphum

flava; Sipha

floccosus; Geopemphigus

Geopemphigus

floccosus

gossypii; Aphis

graminum; Schizaphis

gravicornis; Dactynotus

helichrysi; Brachycaudus

hippophaes javanicus; Capitophorus

hirsuta; Dryopeia, Tetraneura

Hyalomyzus

jussiaeae 
Hyperomyzus lactucae

Hysteroneura setariae

illinoisensis; Aphis

jussiaeae; Hyalomyzus

kahawaluokalani; Melanocallis

lactucae; Hyperomyzus

Lipahis

pseudobrassicae

luteum; Macrosiphum (Sitobion)

Macrosiphum

euphorbiae

luteum

mesosphaeri

rosae

salviae

Macrosiphum (Sitobion)

luteum

salviae

maidis; Rhopalosiphum

Melanocallis

kahawaluokalani

mesosphaeri; Macrosiphum

Microparsus

olivei

minor; Capitophorus (Pentatrichopus)

Myzus

ornatus

persicae

portulacae

Myzus (Nectarosiphon)

persicae

Nectarosiphon

persicae

Neomyzus

circumflexum

nerii; Aphis

nigriabdominalis; Schizoneura, Tetraneura

nigronervosa; Pentalonia nymphaeae; Rhopalosiphum

olivei; Microparsus

orchidearum; Cerataphis

Oregma

panicola

ornatus; Myzus

Ovatus

crataegarius

padi; Aphis, Rhopalosiphum

panicola; Oregma

Pentalonia

nigronervosa

persicae; Myæus (Nectarosiphon)

Picturaphis

brasiliensis

puertoricensis

porosum; Rhodobium

portulacae; Myzus

Prociphilus

erigeronensis

pseudobrassicae; Lipahis

puertoricensis; Picturaphis

Rhodobium

porosum

Ropalosiphum

maidis

nymphaeae

padi

rufiabdominalis

subterraneum

rosae; Macrosiphum

rufiabdominalis; Rhopalosiphum,

Toxoptera

salviae; Macrosiphum (Sitobion)

Schizaphis

graminum

Schizoneura

nigriabdominalis

setariae; Hysteroneura

Sipha

flava 
Siphocoryne splendens

Sitobion luteum salviae solani; Aulacorthum spiraecola; Aphis splendens, Siphocoryne subterranea; Cerosipha subterraneum; Rhopalosiphum
Tetraneura nigriabdominalis hirsuta

Toxoptera aurantiae rufiabdominalis tujafilina; Cinara Tychea erigeronensis variabilis; Cerataphis

\section{INDEX TO GENERA AND SPECIES OF PLANTS'}

Generic names printed in boldface type, specific and common names in roman type; synonyms are in italics.

acerifolia; Anoda

acuta; Sida

adenanthus; Phaseolus

Adenostema

verbesina

aduncum; Piper

aethiopica; Zantedeschia

Ageratum

conyzoides

albiflora; Gesneria, Pentarhaphi

alliodora; Cerdana, Cordia

americana; Hyptis, Mammea

Waltheria

Amphiglottis

secunda

Andropogon

bicornis

anethifolium; Chrysanthemum

angustifolia; Isachne, Jussiaea

Anoda

acerifolia

Anthocephalus

cadamba

antillanum; Calophyllum

arborea; Cyathea

arboreum; Dendropanax

Arundo
donax
Asclepias
curassavica
nivea
Axonopus
compressus
bambos; Bambusa
Bambusa
bambos
vulgaris
basilicum; Ocimum
batatas; Ipomoea
berberis; Myrcia
berteroanus; Sporobolus
bicolor; Tournefortia
bicornis; Andropogon
Bidens
pilosa
reptans
blumei; Coleus
Boerhaavea
coccinea
Boerhaavia
diffusa




\section{Borreria ocimoides \\ Brachyramphus intybaceus \\ brasiliense; Calophyllum cacao; Theobroma cadamba, Anthocephalus cajan; Cajanus \\ Cajanus cajan indicus \\ Calliandra surinamensis \\ Callisia repens \\ Calophyllum antillanum brasiliense \\ Calotropis camara; Lantana campanulata; Spathodea canadensis; Conyza capitata; Hyptis caracu; Xanthosoma \\ Carica papaya carolinensis; Pluchea carpinifolia; Sida \\ Cassia occidentalis tora cathartica; Cracca caudatus; Cosmos \\ Cecropia peltata celosia; Iresine}

Celosia nitida ceratonia; Lomoplis, Mimosa Cerdana alliodora
Cestrum laurifolium macrophyllum

chilensis; Salix

Chrysanthemum anethifolium

ciliata; Dichromena, Galinsoga ciliatum; Solanum cinerea; Cracca, Tephrosia, Vernonia

Cirsium mexicanum

Cissampelos pareira

Cissus sicyoides

Citrus sinensis

Clerodendron fragans

Clibadium erosum

Clusia gundlachii coccigera; Malpighia coccinea; Boerhaavea, Emilia, Ixora, Salvia

Coccoloba microstachya uvifera Coccolobis obtusifolia coelestris; Commelina Coffea robusta Coix lachryma-jobi

Coleus blumei

Commelina coelestris 
diffusa

longicaulis

compressus; Axonopus

conjugatum; Paspalum

Conyza

canadensis

conyzoides; Ageratum

Cordia

alliodora

cordifolia; Mikania

coronarium; Hedychium

Cosmos

caudatus

Costus

cylindricus

Cracca

cathartica

cinerea

crispa; Mentha

Crossandra infundibuliformis

Cucumis

sativus

cumingiana; Triplaris

curassavica; Asclepias

Cyathea

arborea

cylindricus; Costus

Cyperus

ferax

odoratus

polystachyos

rotundus

dahlia

decumbens; Hypoxis

Dendropanax

arboreum

Desmodium

densiflorum; Persicaria

Dichromena

ciliata
Didymopanax

gleasonii

morototoni

Dieffenbachia

seguine

diffusa; Boerhaavia, Commelina;

Iresine

Dilomilis

montana

Ditremexa

occidentalis

Dolichos

lablab

dolicholepsis; Eupatorium

donax; Arundo

Duggena

hirsuta

eetueldeanus; Hibiscus

Elephantopus

mollis

scaber

Emelista

tora

Emilia

coccinea

Epidendrum

prismatocarpum

secundum

erecta; Hamelia

Eriochloa

punctata

erosum; Clibadium

Eugenia

malaccensis

Eupatorium

dolicholepis

odoratum

faba; Vicia

fallax; Myrcia

fasciculatum; Panicum

ferax; Cyperus 
ferruginea; Rapanea

florulentus; Rubus

fragans; Clerodendron

Fragaria

furcellatus; Hibiscus

Galinsoga

ciliata

Gesneria

albiflora

gleasonii; Didymopanax

Gnaphalium

portoricense

Gonzalagunia

spicata

grandiflorus; Malvaviscus

guadalupensis; Melothria

guara; Guarea

Guarea

guara

trichilioides

Guettarda

scabra

guilfoylei; Nothopanax, Polyscias

gundlachii; Clusia

Gynura

haemantha; Tabebuia

Hamelia

erecta

Hedychium

coronarium

Hibiscus

eetweldeanus

furcellatus

rosa-sinensis

hirsuta; Duggena

Hypoxis

decumbens

Hyptis

americana

capitata

pectinata

spicigera
Ichnanthus

pallens

Hex

macfadyenii

indica; Lagerstroemia, Waltheria indicus, Cajanus

infundibuliformis; Crossandra

inga; Inga

Inga

inga

vera

intybacea; Lactuca

intybaceus; Brachyramphus

Ipomoea

batatas

Iresine

celosia

diffusa

Isachne

angustifolia

Ixora

coccinea

Jambosa

malaccensis

Jussiaea

augustifolia

krugii, Plumiera

lablab; Dolichos

Lablab

niger

lachryma-jobi; Coix

Lactuca intybacea

Lagerstroemia indica

lamarckiana; Trema

lancifolia; Saggitaria

Lantana

camara

lathyroides; Macroptilium, Phaseotus

laurifolium; Cestrum 
laxum; Panicum

Lepidiuma virginicum

Leptilon pusillum

lobata; Urena

Lomoplis

ceratonia

longicaulis; Commelina

Ludwigia octovaluis

lunatus; Phaseolus

macarthurii; Ptychosperma

macfadyenii; $\mathbf{H e x}$

macrophyllum; Cestrum

Macroptilium

lathyroides

major; Plantago

malaccensis; Eugenia, Jambosa

Malpighia coccigera

Malvaviscus grandiflorus

Mammea

americana

marginatum; Piper

Melothria

guadalupensis

Mentha

crispa

nemorosa

micrantha; Symplocos, Trema

microstachya; Coccoloba

Mikania

cordifolia

pachyphylla

Mimosa

ceratonia

mexicanum; Cirsium

mollis; Elephantopus

montana, Dilomilis, Octadesmia

morototoni; Didymopanax
Musa

paradisiaca

sapientum

Myrcia

berberis

fallax

splendens

nemorosa; Mentha

Nerium

oleander

nervosa; Rhynchospora

niger, Lablab

nigrum; Xanthosoma

nitida; Celosia

nivea; Asclepias

nobilis; Sanchezia

Nothopanax

guilfoylei

obtusa, Plumiera

obtusifolia; Coccoloba

occidentalis; Cassia, Ditremexa,

Salvia

ocimoides; Borreria

Ocimum

basilicum

Octadesmia

montona

octovalvis; Ludwigia

odorata; Osmia, Pluchea

odoratum; Eupatorium

odoratus; Cyperus

oleander, Nerium

oleracea; Portulaca

oleraceus; Sonchus

Osmanthus

Osmia

odorata

pachyphylla; Mikania

pallens; Ichnanthus

Panicum

fasciculatum 


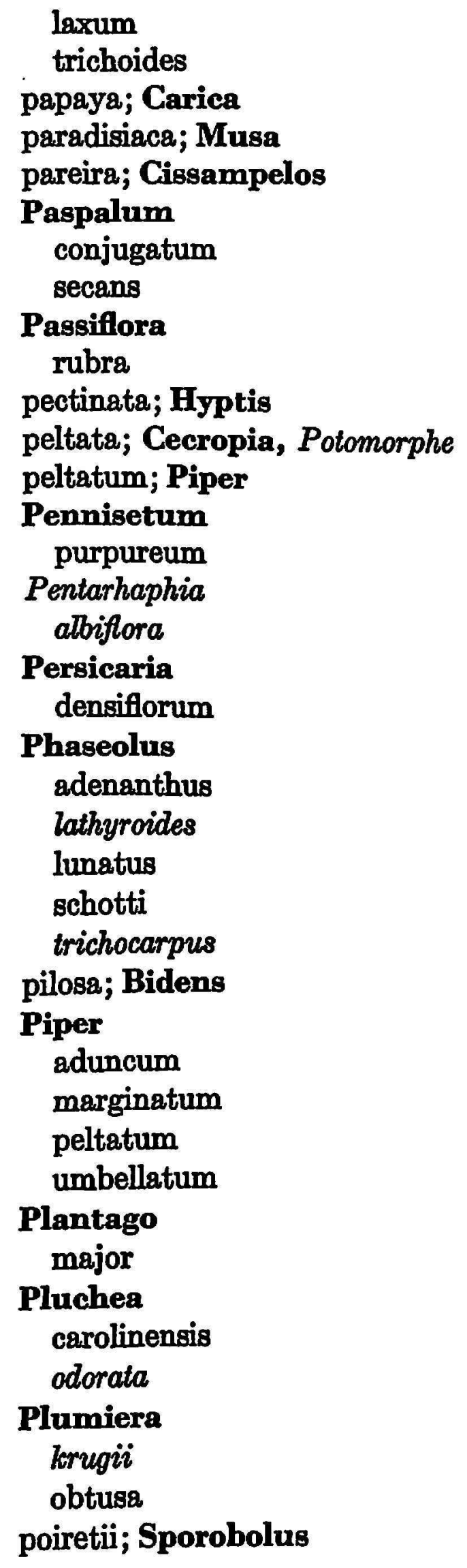

laxum

trichoides

papaya; Carica

paradisiaca; Musa

pareira; Cissampelos

Paspalum

conjugatum

secans

Passiflora

rubra

pectinata; Hyptis

peltata; Cecropia, Potomorphe

peltatum; Piper

Pennisetum

purpureum

Pentarhaphia

albiflora

Persicaria

densiflorum

Phaseolus

adenanthus

lathyroides

lunatus

schotti

trichocarpus

pilosa; Bidens

Piper

aduncum

marginatum

peltatum

umbellatum

Plantago

major

Pluchea

carolinensis

odorata

Plumiera

krugii

obtusa

poiretii; Sporobolus

Polygonum

portoricensis

Polyscias

guilfoylei

polystachyos; Cyperus

portoricense; Gnaphalium

portoricensis; Polygonum

Portulaca

oleracea

Potomorphe

peltata

prismatocarpum; Epidendrum

Ptychosperma

macarthurii

punctata; Eriochloa

purpureum; Pennisetum

pusillum; Leptilon

Rapanea

ferruginea

repens, Callisia

reptans; Bidens

rhombifolia; Sida

Rhychospora

nervosa

robusta; Coffea

Rosa

rosaefolius; Rubus

rosa-sinensis; Hibiscus

rutundus; Cyperus

rubra; Passiflora

Rubus

florulentus

rosaefolius

Saggitaria

lancifolia

Salix

chilensis

Salvia

coccinea

occidentalis

splendens 
Sanchezia

nobilis

sapientum; Musa

sativus; Cucumis

scaber; Elephantopus

scabra; Guettarda

schotti; Phaseolus

seaforthianum; Solanum

secans; Paspalum

secunda; Amphiglottis

secundum; Epidendrum

seguine; Dieffenbachia

senna; Tephrosia

sicyoides; Cissus

Sida

acuta

carpinifolia

rhombifolia

sinensis; Citrus

Solanum

ciliatum

seaforthianum

torvum

Sonchus

oleraceus

Spathodea

campanulata

spicata; Gonzalagunia

spicigera; Hyptis

splendens; Myrcia; Salvia

Sporobolus

berteroanus

poiretii

surinamensis; Calliandra

Symplocos

micrantha

Tabebuia

haemantha

Tephrosia

cinerea

senna
Theobroma cacao

Thuja

tora; Cassia, Emelista

torvum; Solanum

Tournefortia

bicolor

Trema

lamarckiana

micrantha

trichilioides; Guarea

trichocarpus; Phaseolus

trichoides; Panicum

Triplaris

vumingiana

Turnera

ulmifolia

ulmifolia; Turnera

umbellatum; Piper

Urena

lobata

uvifera; Coccoloba

vera; Inga

verbesina; Adenostema

Vernonia

cinerea

Vicia

faba

violaceum; Xanthosoma

virginicum, Lepidium

Vitis

vulgaris; Bambusa

Waltheria

americana

indica

Xanthosoma

caracu

nigren

violaceum

Zantedeschia

aethiopica 


\section{HOST PLANT INDEX AND APHID ASSOCIATION}

Botanical plant names are in bold face type, common names in roman; botanical plant names in synonymy in italics; insect names also in italics. This is a complete index which includes the host plants and aphids mentioned in Technical Paper 37, published by the Agricultural Experiment Station in September 1963, as well as the host plants and aphids discussed in this publication. Names preceded by an asterisk indicate new host plant records not mentioned in Tech. Paper No. 37 or a new aphid record for the host plant.

Abelmoschus esculentus

Aphis gossypii

Actinophloeus macarthurii

(see Ptychosperma macarthurii)

*Adenostema verbesina Aphis gossypii

*Ageratum conyzoides Myzus ornatus

Amaranthus gangeticus var. tricolor

Macrosiphum euphorbiae

Amphiglottis secunda (see Epidendrum secundum)

Anachelium cochleatum (see Epidendrum cochleatum)

Andira inermis Aphis craccivora

Andropogon bicornis

Hysteroneura setariae

Annona muricata

Aphis spiraecola

Toxoptera aurantiae

*Anoda acerifolia Aphis gossypii

*Anthocephalus cadamba

Aphis gossypii

Aphis spiraecola

Toxoptera aurantiae

Apium graveolens

(see Arracacia xanthorriza)
Arundo donax

*Hysteroneura setariae

Rhopalosiphum maidis

Arracacia xanthorriza =

(Apium graveolens)

Nectarosiphon persicae

Asclepias curassavica

Aphis gossypii

Aphis nerii

Asclepias nivea

Aphis nerii

Aster sp

Dactynotus ambrosiae

Axonopus compressus

Hysteroneura setariae

*Rhopalosiphum rufoabdominalis

Bambusa bambos =

(Bambusa vulgaris)

Hysteroneura setariae

*Rhopalosiphum padi

Bambusa vulgaris

(see Bambusa bambos)

Barringtonia asiatica

Toxoptera aurantiae

Bean vine

Picturaphis brasitiensis

Bidens cynapiifolia

Aphis coreopsidis

Dactynotus ambrosiae

Bidens pilosa

Acyrthosiphon bidenticola 
Aphis coreopsidis

Aphis gossypii

Aulacorthum circumflexum

Aulacorthum solani

Dactynotus ambrosiae

Hysteroneura setariae

Myzus ornatus

*Myzus (Nectarosiphon) persicae

*Bidens reptans

Aphis coreopsidis

Bidens urbanii

Dactynotus ambrosiae

Bignonaceous vine

Toxoptera aurantiae

Bocconia frutescens

Aulacorthum circumflexum

Myzus (Nectarosiphon) persicae

Boerhaavea coccinea

(see Boerhaavia diffusa)

*Boerhaavia diffusa $=$

(Boerhaavea coccinea)

Hysteroneura setariae

Boerhaavia erecta

Aphis coreopsidis

Aphis spiraecola

*Borreria ocimoides

Aphis craccivora

Borreria verticillata

Aphis spiraecola

Myzus (Nectarosiphon) persicae

Bougainvillea spectabilis

Aphis spiraecola

Brachyramphus intybaceus

(see Lactuca intybacea)

Brassica integrifolia

Amphorophora commelinensis

Aulacortum solani

Brevicoryne brassicae

Lipaphis pseudobrassicae

Myzus (Nectarosiphon) persicae
Brassica olerace

Brevicoryme brassicae

Myzus (Nectarosiphon) persicae

Bryophyllum pinnatum

Aphis gossypii

Bursera simaruba =

(Elaphrium simaruba)

Aphis spiraecola

Cajanus cajan $=$

(Cajanus indicus)

Aphis craccivora

Aphis gossypii

Aphis spiraecola

Cajanus indicus

(see Cajanus cajan)

Caladium colocasia

(see Colocasia esculenta)

*Calliandra surinamensis

Toxoptera aurantiae

Callisia monandra

Amphorophora commelinensis

*Callisia repens

Amphorophora commelinensis

Calophyllum antillanum

(see Calophyllum brasiliense)

Calophyllum brasiliense $=$

(Calophyllum antillanum)

Toxoptera aurantiae

Calotropis procera

Aphis nerii

Canavalia maritima

Aphis craccivora

Capparis flexuosa

Aphis craccivora

Capraria biflora

Aphis gossypii

Myzus (Nectarosiphon) persicae

Capsicum anuum

Aphis gossypii

Myzus (Nectarosiphon) persicae 
Capsicum baccatum

Myzus (Nectarosiphon) persicae

Capsicum sp.

Myzus (Nectarosiphon) persicae

*Carica papaya

Aphis coreopsidis

Aphis nerii

Aphis spiraecola

Casearia arborea

Toxoptera aurantiae

Casearia decandra Aphis spiraecola

Casearia sylvestris

Toxoptera aurantiae

Cassia obtusifolia = (Emelista tora)

*Aphis gossypii

Macrosiphum ambrosiae

Cassia occidentalis $=$

(Ditremexa occidentalis)

Aphis gossypii

Cassia tora $=$

(Emelista tora)

Aphis gossypii

Catharanthus roseus

Aphis gossypii

Aphis spiraecola

Cattleya luddemanniana $(=C$. speciosissima)

Toxoptera aurantiae

Cecropia peltata

Aphis gossypii

Aphis spiraecola

*Celosia nitida

Myzus ornatus

Myzus (Nectarosiphon) persicae

*Celosia sp.

Aphis spiraecola

Cerdana alliodora

(see Cordia alliodora)

Cestrum laurifolium

Aphis spiraecola
Cestrum macrophyllum

*Aphis gossypii

*Aphis spiraecola

Aulacorthum solani

Toxoptera aurantiae.

Chalcas exotica

(see Murraya exotica)

Chenopodium ambrosioides Myzus (Nectarosiphon) persicae

Chinese fan palms

Cerataphis variabilis

Chloris inflata

Hysteroneura setariae

Chrysanthemum anethifolium

Dactynotus ambrosiae

*Chrysanthemum sp. Aphis spiraecola

Cinnamomum burmanni

Toxoptera aurantiae

Cirsium mexicanum

Aphis gossypii

Aphis spiraecola

Capitophorus elaeagni

Dactynotus ambrosiae

*Hyperomyzus lactucae

*Cissampelos pareira

Aphis spiraecola

Cissus sycyoides

Aphis illinoisensis

Cithraexylum fruticosum

Aphis spiraecola

Citrus aurantium

Aphis spiraecola

Toxoptera aurantiae

Citrus limonia

Aphis spiraecola

Toxoptera aurantiae

Citrus maximum

Aphis spiraecola

Toxoptera aurantiae 
Citrus sinensis

Aphis spiraecola

Toxoptera aurantiae

Citrus sp.

Aphis spiraecola

Pentalonia nigronervosa

Cleome gynandra

Myzus (Nectarosiphon) persicae

Clerodendron fragans

Myzus (Nectarosiphon) persicae

*Aphis gossypii

Clibadium erosum

Aphis coreopsidis

Aphis gossypii

*Aphis spiraecola

*Dactynotus ambrosiae

* Macrosiphum (Sitobion) salviae

*Myzus ornatus

Clusia gundlachi

Toxoptera aurantiae

Cocos nucifera

Cerataphis variabilis

Coccoloba borinquensis

Toxoptera aurantiae

Coccoloba diversifolia

Aphis gossypii

Coccoloba microstachya $=$

(Coccolobis obtusifolia)

Aphis craccivora

*Toxoptera aurantiae

Coccoloba pirifolia

Toxoptera aurantiae

Coccoloba uvifera

Aphis craccivora

*Aphis gossypii

Toxoptera aurantiae

Coccoloba sp.

Aphis craccivora

Coccolobis obtusifolia

(see Coccoloba microstachya)

Coffea arabiga

Toxoptera aurantiae
*Coffea robusta

Aphis gossypii

Coix lachryma-jobi

Rhopalosiphum maidis

*Coleus blumei

Myzus ornatus

Colocasia esculenta $=$ (Caladium colocasia) Aphis gossypii

Commelina coelestris Amphorophora commelinensis Aulacorthum circumflexum

Commelina diffusa $=$ (Commelina longicaulis) Amphorophora commelinensis

* Oregma panicola

Commelina longicaulis. (see Commelina diffusa)

Commelina sp. Aphis gossypii

Conyza apurensis $=$ (Leptilon chinense)

Dactynotus gravicornis

Conyza canadensis $=$ (Leptilon pusillum)

Dactynotus erigeronensis

Dactynotus gravicornis

Cordia alliodora $=$

(Cerdana alliodora)

Aphis spiraecola

Toxoptera aurantiae

Cordia nitida

Aphis spiraecola

* Cosmos caudatus

Aphis coreopsidis

Cosmos sp.

Dactynotus ambrosiae

*Costus cylindricus

Pentalonia nigronervosa

Cracca cathartica

(see Tephrosia senna) 
Cracca cinerea (see Tephrosia cinerea)

Critonia portoricensis Dactynotus ambrosiae

*Crossandra infundibuliformis Aphis gossypii

Crotalaria retusa Aphis gossypii

Crotalaria stipularia Macrosiphum euphorbiae

- Crotalaria striata Aphis gossypii

Croton humilis Aphis spiraecola

Cruciferous plant (unidentified) Myzus (Nectarosiphon) persicae

Cucumis melo Aphis gossypii

Cucumis sativus Aphis gossypii

*Cyathea arborea Aphis gossypii

Cymbopogon citratus Sipha flava

Cyperus odoratus (see Cyperus polystachyos)

Cyperus peruvianus $=$ (Kyllinga peruviana) Carolinaia caricis

*Cyperus polystachyos = (Cyperus odoratus) Carolinaia cyperi

Cyperus rotundus Carolinaia cyperi *Rhopalosiphum padi

Cyperus sp. Carolinaia cyperi

Cyrtospadix bicolor Pentalonia nigronervosa dahlia

Prociphilus erigeronensis
Dahlia pinnata Aphis gossypii Dactynotus ambrosiae *Prociphilus erigeronensis

Dendrobium moschatum Sitobium luterm

*Dendropanax arboreum Toxoptera aurantiae

Desmodium supinum $=$ (Meibomia supina) Microparsus olivei

Dichromena ciliata (see Rhynchospora nervosa)

Dichromena radicans (see Rhynchospora radicans)

*Didymopanax gleasonii Toxoptera aurantiae

Didymopanax morototoni Toxoptera aurantiae

Dieffenbachia seguine *Aphis spiraecola Pentalonia nigronervosa ${ }^{*}$ Rhopalosiphum nymphaeae

Digitaria adscendens $=$ (Digitaria sanguinalis) Hysteroneura setariae Schizaphis graminum Sipha flava

Digitaria decumbens Sipha flava Tetraneura nigriabdominalis

Digitaria sanguinalis (see Digitaria adscendens)

Dilomilis montana $=$

(Octadesmia montana) * Aphis spiraecola Aulacorthum circumflexum Aulacorthum solani Sitobium luterm

Ditremexa occidentalis (see Cassia occidentalis) 
Dolichos lablab

(see Lablab niger)

Duggena hirsuta (see Gonzalagunia spicata)

Echites agglutinata Aphis spiraecola

Eclipta alba Dactynotus ambrosiae

Elaphrium simaruba (see Bursera simaruba)

Elephantopus mollis (see Elephantopus scaber)

"Elephantopus scaber $=$ (Elephantopus mollis) Aulacorthum solani Dactynotus ambrosiae

Eleusine indica

Hysteroneura setariae

Emelista tora

(see Cassia obtusifolia)

Emilia coccinea

Aphis gossypii

Aphis spiraecola

Aulacorthum circumflexum

Macrosiphum euphorbiae

Myzus (Nectarosiphon) persicae

*Tetraneura nigriabdominalis

Emilia sonchifolia

Aulacorthum circumflexum

Hyperomyzus lactucae

Myzus (Nectarosiphon) persicae

Epidendrum cochleatum =

(Anachelium cochleatum)

Cerataphis orchidearum

Sitobium luteum

Epidendrum prismatocarpum Aphis spiraecola

Epidendrum secundum =

(Amphiglottis secunda)

Aphis gossypii

*Aphis spiraecola
Aulacorthum circumflexum

Cerataphis orchidearum

Myzus (Nectarosiphon) persicae

*Toxoptera aurantiae

Epidendrum sp. Sitobion luteum

Erechtites valerianaefolia Acyrthosiphon bidenticola Aphis spiraecola Aulacothum circumflexum Dactynotus ambrosiae Hyperomyzus lactucae

*Eriochloa punctata Rhopalosiphum maidis Rhopalosiphum padi Sipha flava

Erithalis fruticosa Aphis spiraecola

Eugenia borinquensis Aulacorthum solani

Eugenia jambos Toxoptera aurantiae

*Eugenia malaccensis $=$ (Jambosa malacensis) Toxoptera aurantiae

*Eupatorium dolicholepis Aphis spiraecola

Eupatorium odoratum $=$ (Osmia odorata) Aphis gossypii Aphis spiraecola Dactynotus ambrosiae

Eupatorium polyodon Aphis spiraecola Dactynotus ambrosiae

Exostema caribaeum Aphis spiraecola

Ficus pumila Aulacorthum circumflexus

Fleurya aestuans Macrosiphum euphorbiae Myzus (Nectarosiphon) persicae 
Fragaria chiloensis

Capitophorus (Pentatrichopus) minor

Fuirena umbellata

(see Scirpus fuirena)

Furcraea tuberosa Aphis spiraecola

*Galinsoga ciliata Dactynotus ambrosiae Myzus ornatus

Gardenia jasminoides Aphis spiraecola

*Gesneria albiflora $=$ (Pentarhapia albiflora) Toxoptera aurantiae

Gliricidia sepiuma Aphis craccivora

*Gnaphalium portoricense Dactynotus ambrosiae

Gonzalagunia spicata $=$ (Duggena hirsuta) Aphis gossypii Toxoptera aurantiae

Gossypiuma barbadense var. Sea Island Aphis gossypii

Gossypium hirsutum var. punctatum Aphis gossypii

Gossypium sp. Aphis gossypii grass

Hysteroneura setariae green peas Aphis craccivora

Guarea guara (see Guarea trichilioides)

*Guarea trichilioides = (Guarea guara) Toxoptera aurantiae Guazuma guazuma (see Guazuma ulmifolia)
Guazuma ulmifolia $=$ (Guazuma guazuma) Toxoptera aurantiae

Guettarda krugii Aphis spiraecola

*Guettarda scabra Aphis gossypii

Gynura aurantiaca Brachycaudus helichrysi

Habenaria monorrhiza Aulacorthum circumflexum Aulacorthum solani

Hamelia erecta Aphis spiraecola *Toxoptera aurantiae

Hamelia sp. Aphis gossypii Aphis spiraecola

*Hedychium coronarium Pentalonia nigronervosa

Helianthus annuus Dactynotus ambrosiae

Heliconia latispatha Pentalonia nigronervosa

Hemidiodia ocimifolia Aphis gossypii

Hernandia sonora Aphis gossypii

*Hibiscus eetveldeanus Aphis gossypii

*Hibiscus furcellatus Aphis gossypii

Hibiscus rosa-sinensis Aphis coreopsidis Aphis gossypii Toxoptera aurantiae

Holcus sorghum Rhopalosiphum maidis

Hydrocleys nymphoides Rhopalosiphum nymphaeae

*Hypoxis decumbens Rhopalosiphum padi 
Hyptis americana (see Hyptis spicigera)

*Hyptis capitata Macrosiphum (Sitobion) salviae

Hyptis pectinata *Aphis gossypii Macrosiphum mesosphaeri *Macrosiphum (Sitobion) salviae

*Hyptis spicigera = (Hyptis americana) Macrosiphum (Sitobion) salviae

Ichnanthus pallens Oregma panicola

*Iex macfadyenii Aphis coreopsidis Toxoptera aurantiae

Ilex vomitaria Toxoptera aurantiae

Indigofera sp. Aphis craccivora

Indigofera suffiruticosa Aphis craccivora

Inga inga (see Inga vera)

Inga laurina Macrosiphum mesosphaeri

Inga vera $=$ (Inga inga) Macrosiphum (Sitobion) salviae Ipomoea batatas Aphis spiraecola Geopemphigus floccosus Myzus (Nectarosiphon) persicae Ipomoea sp.

Aulacorthum circumflexum

Iresine celosia (see Iresine diffusa)

*Iresine diffusa $=$ (Iresine celosia) Rhopalosiphum padi Toxoptera aurantiae
Iresine sp. Aphis coreopsidis

Isachne angustifolia Oregma panicola

Ixora coccinea Aphis spiraecola

Ixora lutea Aphis spiraecola Jambosa malaccensis (see Eugenia malaccensis)

Jussiaea angustifolia (see Ludwigia octavalvis)

Killinga peruviana (see Cyperus peruvianus)

Lablab niger $=$ (Dolichos lablab) * Aphis craccivora * Aucorthum circumflexum Picturaphis brasiliensis

Lactuca floxidana Aulacorthum circumflexum Aulacorthum solani Dactynotus ambrosiae Myzus ornatus Myzus (Nectarosiphon) persicae

* Lactuca intybacea = (Brachyramphus intybaceus) Aulacorthum circumflexum

Lactuca sativa

Dactynotus ambrosiae Macrosiphum euphoribiae Myzus (Nectarosiphon) persicae

Lagerstroemia indica Aphis spiraecola Melanocallis kahawaluokalani Toxoptera aurantiae

Lagerstroemia speciosa Toxoptera aurantiae

Lantana camara Aphis spiraecola Aulacorthum solani Macrosiphum (Sitobion) salviae 
Lasiacis divaricata

Oregma panicola

Lasiacis maculata $=$

(Lasiacis sorghoidea)

Oregma panicola

Lasciasis sorghoidea (see Lasciasis maculata)

Leonitis nepetaefolia Aphis gossypii

*Lepidium virginicum Myzus (Nectarosiphon) persicae

Leptilon chinense (see Conyza apurensis)

Leptilon pusillum (see Conyza canadensis)

lima bean

Prociphilus erigeronensis

*Lomoplis ceratonia = (Mimosa ceratonia) Macrosiphum (Sitobion) salviae

Ludwigia octovalvis = (Jussiaea angustifolia) Aphis spiraecola Hyalomyzus jussiaea *Myzus ornatus

Lycopersicon esculentum Macrosiphum euphorbiae

*Macroptilium lathyroides = (Phaseolus lathyroides) Aphis craccivora

*Malpighia coccigera Toxoptera aurantiae

Malpighia fucata Aphis spiraecola Toxoptera aurantiae

Malpighia glabra Aphis craccivora

Malpighia punicifolia Aphis spiraecola Toxoptera aurantiae Malvaviscus grandiflorus Aulacorthum cicumflexum
Aulacorthum solani

Toxoptera aurantiae

*Mammea americana Toxoptera aurantiae

Mangifera indica

Aphis spiraecola

Toxoptera aurantiae

Meibomia supina (see Desmodium supinum)

Melanthera confusa Aphis spiraecola Dactynotus ambrosiae

*Melothria guadalupensis Aphis gossypii

*Mentha crispa Ovatus crataegarius

*Mentha nemorosa Myzus ornatus

Mikania cordifolia *Aphis gossypii Aphis spiraecola

*Mikania pachyphylla Aphis coreopsidis

Mimosa ceratonia (see Lomoplis ceratonia) Mint

Ovatus crataegarius

Momordica charantia Aphis gossypii

Montezuma speciosissima Aphis gossypii

Moringa oleifera Aphis spiraecola Myzus (Nectarosiphon) persicae

Murraya exotica $=$ (Chalcas exotica) Aphis spiraecola Toxoptera aurantiae

Musa paradisiaca Pentalonia nigronervosa *Aphis gossypii 
Musa sapientum

Pentalonia nigronervosa

Myrcia berberis

(see Myrcia fallax)

*Myrcia fallax =

(Myrcia berberis)

Toxoptera aurantiae

Myrcia splendens

Toxoptera aurantiae

Nectandra antillana

Toxoptera aurantiae

Nerium oleander

Aphis nerii

Nicotiana tabacum

Myzus (Nectarosiphon) persicae

Nothopanax guilfoyle $=$

(Polyscias guilfoylei)

Aphis spiraecola

Myzus (Nectarosiphon) persicae

Toxoptera aurantiae

Nymphaea caerulea

Rhopalosiphum nymphaeae

*Ocimum basilicum

Myzus ornatus

Octadesmia montana

(see Dilomilis montana)

orange orchid

Aphis spiraecola

Orchids

Cerataphis orchidearum

Sitobion luteum

*Osmanthus sp.

Aphis spiraecola

Myzus (Nectarosiphon) persicae

Osmia odorata

(see Eupatorium odoratum)

Palms

Cerataphis variabilis

Pandorea ricasoliana

Aphis gossypii

Panicum conjugatum

Sipha flava
*Panicum fasciculatum

Sipha flava

*Panicum laxum

Hysteroneura setariae

Panicum maximum

Sipha flava

Panicum trichoides

Oregma panicola

*Hysteroneura setariae

Parthenium hysterophorum

Dactynotus ambrosiae

Paspalum boscianum =

(Paspalum melanospermum)

Sipha flava

*Paspalum conjugatum

Rhopalosiphum padi

Paspalum fimbriatum

Hysteroneura setariae

Macrosiphum euphorbiae

Paspalum melanospermum (see Paspalum boscianum)

Paspalum paniculatum

Hysteroneura setariae

Sipha flava

*Paspalum secans

Hysteroneura setariae

*Passiflora rubra

Rhopalosiphum padi

Paullinia pinnata

Aphis spiraecola

Pennisetum purpureum

Sipha flava

*Rhopalosiphum padi

Pentarhaphia albifiora

(see Gesneria albiflora)

Pepo moschata

Aphis gossypii

Persea gratissima

Aphis gossypii

*Persicaria densiflorum = (Polygonum portoricensis) 
Capitophorus hippophaes javanicus

Persicaria sp. (= Polygonum sp.) Capitophorus hippohaes javanicus Petrea volubilis Aphis gossypii

*Phaseolus adenanthus

Picturaphis puertoricensis

Phaseolus lathyroides (see Macroptilium lathy roides)

Phaseolus lunatus

Aphis craccivora

*Aphis gossypii

Picturaphis brasiliensis

*Phaseolus schottii =

(Phaseolus trichocarpus)

Aphis craccivora

Picturaphis brasiliensis

Phaseolus sp.

Picturaphis brasiliensis

Phaseolus trichocarpus

(see Phaseolus schotti)

Phaseolus vulgaris

Aphis craccivora

Phoebe elongata

Toxoptera aurantiae

Physalis pubescens

- Myzus (Nectarosiphon) persicae

Pictetia aculeata

Aphis craccivora

Piper aduncum

Aphis gossypii

Aulacorthum circumflexum

Aulacorthum solani

Toxoptera aurantiae

*Piper marginatum

Toxoptera aurantiae

Piper peltatum $=$

(Pothomorphe peltata)

Aphis gossypii
Aphis spiraecola

Dactynotus ambrosiae

Piper umbellatum

Aphis gossypii

Pithecellobium dulce

Aphis craccivora

Plantago sp.

Aulacorthum solani

Plantago major

Aphis gossypii

Aulacorthum solani

Carolinaia cyperi

Myzus ornatus

Pleurothalis near ruscifolia

Sitobion luteum

Pluchea carolinensis $=$

(Pluchea odorata)

Aphis gossypii

Dactynotus ambrosiae

Pluchea odorata

(see Pluchea carolinensis)

Pluchea purpurascens

Dactynotus ambrosiae

Plumiera krugii

(see Plumiera obtusa)

*Plumiera obtusa $=$

(Plumiera krugii)

Aphis spiraecola

Poliantes tuberosa

Myzus (Nectarosiphon) persicae

Polygonum portoricensis (see Persicaria densifforum)

Polyscias guilfoylei

(see Nothopanax guilfoylei)

Polystachya extinctoria $=$

(Polystachya minuta)

Cerataphis orchidearum

Polystachya minuta

(see Polystachya extinctoria)

*Portulaca oleracea

Aphis craccivora 
Pothomorphe peltata (see Piper peltatum)

Psidium guajava Aphis gossypii Aphis spiraecola

Psychotria grandis Aphis gossypii Aulacorthum solani

Ptychosperma macarthurii $=$ (Actinophloeus macarthurii) Cerataphis variabilis

Randia mitis Aphis spiraecola Toxoptera aurantiae

Rapanea ferruginea Toxoptera aurantiae Rapanea guianensis Toxoptera aurantiae Rauvolfia nitida $=$ (Rauvolfia tetraphylla) Aphis spiraecola

Rauvolfia viridis $=$ (Rauwolfia lamarckii) Aphis spiraecola

Rauwolfia lamarckii (see Rauvolfia viridis) Rauwolfia tetraphylla (see Rauvolfia nitida) *Rhynchospora nervosa = (Dichromena ciliata) Carolinaia cyperi

Rhynchospora radicans $=$ (Dichromena radicans)

Carolinaia cyperi Hysteroneura setariae

*Rhynchospora sp. Rhopalosiphum padi

Rosa sp.

*Aphis spiraecola

* Macrosiphum euphorbiae

* Macrosiphum rosae
Rhodobium porosum

Toxoptera aurantiae

*Rubus florulentus

Toxoptera aurantiae

*Rubus rosaefolius

Aulacorthum circumflexum Aulacorthum solani

Ruellia coccinea Aphis gossypii

Ruellia tweediana Aphis gossypii

Rumex crispus Myzus (Nectarosiphon) persicae

Rynchelytrum repens $=$ (Tricholaena repens)

Hysteroneura setariae

Rynchospora cyperoides Carolinaia caricis

Sabinea punicea Aphis craccivora

Saccharum officinarum Sipha flava

*Saggitaria lancifolia Aphis gossypii

*Salix chilensis Aphis spiraecola Myzus ornatus

Salvia coccinea Myzus ornatus Macrosiphum (Sitobion) salviae

Salvia occidentalis Aphis gossypii * Macrosiphum (Sitobion) salviae Toxoptera aurantiae

*Salvia splendens Macrosiphum (Sitobion) saltiae Myzus ornatus

*Sanchezia nobilis Aphis gossypii

Scirpus fuirena $=$ (Fuirena umbellata) Carolinaia caricis 
scrophulariaceous splant

Aulacorthum solani

Sechium edule

Aphis gossypii

Sencio confusus

Aphis spiraecola

Dactynotus ambrosiae

Myzus (Nectarosiphon) persicae

Senecioides cinerea $=$

(Vernonia cinerea)

Aphis spiraecola

Serjania polyphylla

Aphis spiraecola

Setaria geniculata

Hysteroneura setariae

*Sida acuta $=$

(Sida carpinifolia)

Aphis gossypii

Sida carpinifolia

(see Sida acuta)

*Sida rhombifolia

Aphis gossypii

Sida sp.

Aphis coreopsidis

Aphis gossypii

Aphis spiraecola

Aulacorthum solani

*solanaceous vine

Myzus (Nectarosiphon) persicae

Solanum caribaeum

(see Solanum nodiflorum)

*Solanum ciliatum

Aulacorthum circumflexum

Solanum melongena

Macrosiphum euphorbiae

Solanum nigrum

(see Solanum nodiflorum)

Solanum nodiflorum $=$

(Solanum nigrum)

(Solanum caribaeum)
Aphis gossypii

Myrus (Nectarosiphon) persicae

*Solanum seaforthianum

Myzus (Nectarosiphon) persicae

Solanum torrum

Aphis gossypii

*Dactynotus ambrosiae

Solanum weadlandii

Aphis gossypii

Sonchus asper

Dactynotus ambrosiae

Hyperomyzus lactucae

Myzus ornatus

Sonchus oleraceus

Aphis coreopsidis

Aphis spiraecola

Dactynotus ambrosiae

Hyperomyzus lactucae

* Myzus (Nectarosiphon) persicae

Spathodea campanulata

Aphis gossypii

Aphis spiraecola

Myzus ornatus

Spondias cirouella

Aphis spiraecola

Spondias mombin

Aphis spiraecola

Toxoptera aurantiae

Spondias purpurea

Aphis spiraecola

Myzus (Nectarosiphon) persicae

Sporobolus berteroanus

(see Sporobolus poiretii)

*Sporobolus poiretii =

(Sporobolus berteroanus)

Hysteroneura setariae

Stachytarpheta jamaicensis $=$

(Valerianoides jamaicensis)

Aphis gossypii

Stigmaphyllon tomentosum

Aphis craccivora 
Swinglea glutinosa

Aphis spiraecola

*Symplocos micrantha

Toxoptera aurantiae

*Tabebuia haemantha

Toxoptera aurantiae

Tabebuia heterophylla = (Tabebuia pallida)

Aphis craccivora

Aphis gossypii

*Toxoptera aurantiae

Tabebuia pallida

(See Tabebuia heterophylla)

Tamarindus indicus Aphis craccivora

Tectona grandis Myzus (Nectarosiphon) persicae

*Tephrosia cinerea $=$ (Cracca cinerea)

Aphis craccivora

*Tephrosia senna = (Cracca cathartica) Aphis craccivora

*Theobroma cacao Toxoptera aurantiae

Thuja orientalis Cinara tujafilina

Thunbergia alata Aphis gossypii

Tithonia diversifolia Aphis gossypii Aphis spiraecola Dactynotus ambrosiae

Torrubia fragans Toxoptera aurantiae

*Tournefortia bicolor Aphis gossypii Myzus (Nectarosiphon) persicae

Tournefortia hirsutissima Aphis gosspii
*Trema lamarckiana

Dactynotus ambrosiae

Macrosiphum (Sitobion) salviae

* Trema micrantha

Aphis spiraecola

Dactynotus ambrosiae

Tricholaena repens (see Rynchelytrum repens)

*Triplaris cumingiana Aphis gossypii

*Turnera ulmifolia Aphis gossypii

Urena lobata

*Aphis spiraecola Aulacorthum circumflexum Aulacorthum solani

Urena trilobata Aphis gossypii

Valerianoides jamaicensis (see Stachytarpheta jamaicensis)

Vanilla planifolia Cerataphis orchidearum

Varronia corymbosa Dactynotus ambrosiae

Verbesina alata Dactynotus ambrosiae

Vernonia cinerea (see Senecioides cinerea)

*Vicia faba Aphis craccivora

Vitis vinifera Aphis illinoisensis

*Walking iris Aphis spiraecola

Waltheria americana (see Waltheria indica)

*Waltheria indica = (Waltheria americana) Dactynotus ambrosiae 
Wedelia trilobata

Aulacorthum circumflexum

*Xanthosoma caracu

Pentalonia nigronervosa

Xanthosoma nigum $=$

(Xanthosoma violaceum)

Aphis gossypii

Xanthosoma sp. Aphis gossypii

Pentalonia nigronervosa

Xanthosoma violaceum

(see Xanthosoma nigrum)
Xolisma rubiginosa

Dactynotus ambrosiae

Toxoptera aurantiae

*Zantedeschia aethiopica

Aulacorthum solani

Zanthoxylum flavum Toxoptera aurantiae

Zanthoxylum monophyllum Aphis spiraecola

Zea mays

Hysteroneura setariae

Rhopalosiphum maidis 